\title{
THE BROWNIAN LIMIT OF SEPARABLE PERMUTATIONS
}

\author{
By Frédérique BASSINO*, MATHILde Bouvel ${ }^{\dagger, 2}$, VALENTIN FÉrAY ${ }^{\dagger}$, \\ LUCAS GERIN ${ }^{\ddagger}$ AND ADELINE PIERROT ${ }^{\S}$ \\ Université Paris 13*, Universität Zürich ${ }^{\dagger}$, École Polytechnique ${ }^{\ddagger}$ and \\ Université Paris-Sud ${ }^{\S}$
}

We study uniform random permutations in an important class of patternavoiding permutations: the separable permutations. We describe the asymptotics of the number of occurrences of any fixed given pattern in such a random permutation in terms of the Brownian excursion. In the recent terminology of permutons, our work can be interpreted as the convergence of uniform random separable permutations towards a "Brownian separable permuton".

\section{CONTENTS}

1. Introduction . . . . . . . . . . . . . . . . . . . . . . . 2135

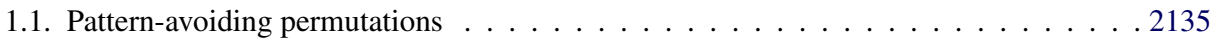

1.2. Overview of our results . . . . . . . . . . . . . . . . . . . . 2138

1.3. Interpretation of our main result in terms of permutons . . . . . . . . . . . . 2140

1.4. Perspectives . . . . . . . . . . . . . . . . . . . . . . . . 2142

1.5. Outline of the paper . . . . . . . . . . . . . . . . . . . . 2143

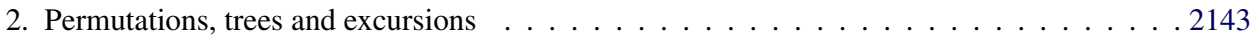

2.1. Basics on trees . . . . . . . . . . . . . . . . . . . . . . . . 2143

2.2. Separable permutations and Schröder trees . . . . . . . . . . . . . . . . 2145

2.3. Contours and excursions . . . . . . . . . . . . . . . . . . . . . 2148

2.4. Extracting trees and permutations from excursions . . . . . . . . . . . . 2151

2.5. Measurability issues . . . . . . . . . . . . . . . . . . . . . . . 2154

3. The variables $\Lambda_{\pi}$ : Definition, properties and proof schema of the main result $\ldots \ldots \ldots 2155$

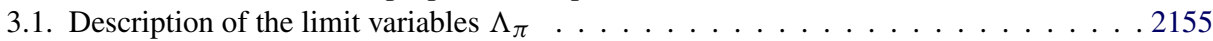

3.2. The leaf distribution function of a tree . . . . . . . . . . . . . . . . . . 2157

3.3. Reformulation of the main theorem . . . . . . . . . . . . . . . . . . . . 2159

3.4. Outline of the proof . . . . . . . . . . . . . . . . . . . 2160

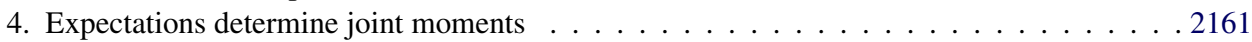

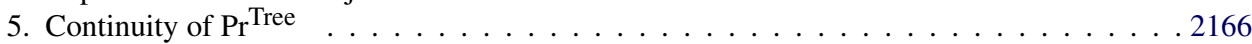

6. Signs are asymptotically balanced and independent . . . . . . . . . . . . . 2168

7. Conclusion of the proof . . . . . . . . . . . . . . . . . . . . . . . . 2174

8. Permuton interpretation of our main result f . . . . . . . . . . . . . . . . 2177

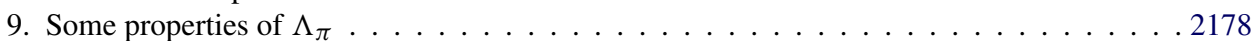

Received April 2016; revised February 2017.

${ }^{1}$ Supported by a Swiss-French PHC Germaine de Stael grant (with project number 2015-09).

${ }^{2}$ Supported by a Marie Heim-Vögtlin grant of the Swiss National Science Foundation. MSC2010 subject classifications. 60C05, 05A05.

Key words and phrases. Permutation patterns, Brownian excursion, permutons. 
9.1. Computing expectation and other moments of $\Lambda_{\pi} \ldots \ldots \ldots$. . . . . . . . . 2179

9.2. $\Lambda_{\pi}$ is not deterministic . . . . . . . . . . . . . . . . . . . . . 2181

Appendix: Useful facts on the Brownian excursion . . . . . . . . . . . . . . . . . . 2184

Acknowledgements . . . . . . . . . . . . . . . . . . . . . . . . . 2187

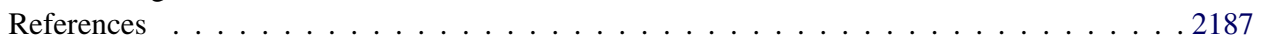

1. Introduction. The aim of this article is to study the asymptotic properties of an important class of pattern-avoiding permutations: the separable permutations. Our main result is the description of the asymptotics in $n$ of the number of occurrences of any fixed given pattern in a uniform separable permutation of $n$ elements.

1.1. Pattern-avoiding permutations. We first give some definitions. For any $n$, the set of permutations of $[n]:=\{1,2, \ldots, n\}$ is denoted by $\mathfrak{S}_{n}$. We write permutations of $\mathfrak{S}_{n}$ in one-line notation as $\sigma=\sigma_{1} \sigma_{2} \ldots \sigma_{n}$. For a permutation $\sigma$ in $\mathfrak{S}_{n}$, the size $n$ of $\sigma$ is denoted by $|\sigma|$. For $\sigma \in \mathfrak{S}_{n}$, and $I \subset[n]$ of cardinality $k$, let $\operatorname{pat}_{I}(\sigma)$ be the permutation of $\mathfrak{S}_{k}$ induced by $\left\{\sigma_{i}, i \in I\right\}$. For example, for $\sigma=65831247$ and $I=\{2,5,7\}$ we have

$$
\operatorname{pat}_{\{2,5,7\}}(6 \overline{5} 83 \overline{1} 2 \overline{4} 7)=312
$$

since the values in the subsequence $\sigma_{2} \sigma_{5} \sigma_{7}=514$ are in the same relative order as in the permutation 312. A permutation $\pi=\operatorname{pat}_{I}(\sigma)$ is a pattern involved in $\sigma$, and the subsequence $\left(\sigma_{i}\right)_{i \in I}$ is an occurrence of $\pi$ in $\sigma$.

All along this paper, we use letter $\sigma$ for a (large) permutation of size $n$, and letter $\pi$ for a pattern of size $k \leq n$. We denote by $\widetilde{\text { occ }}(\pi, \sigma)$ the proportion of occurrences of a pattern $\pi$ in $\sigma$. More formally,

$$
\widetilde{\operatorname{occ}}(\pi, \sigma)=\frac{1}{\left(\begin{array}{c}
n \\
k
\end{array}\right)} \operatorname{card}\left\{I \subset[n] \text { of cardinality } k \text { such that } \operatorname{pat}_{I}(\sigma)=\pi\right\} .
$$

Equivalently, $\widetilde{\mathrm{occ}}(\pi, \sigma)$ is the probability to have pat $_{I}(\sigma)=\pi$ if $I$ is randomly and uniformly chosen among the $\left(\begin{array}{l}n \\ k\end{array}\right)$ subsets of $[n]$ with $k$ elements. If $|\pi|>|\sigma|$, we set conveniently $\widetilde{\text { occ }}(\pi, \sigma)=0$.

We say that $\sigma$ avoids $\tau$ if there is no occurrence of $\tau$ in $\sigma$, that is, $\widetilde{\operatorname{occ}}(\tau, \sigma)=0$. For any (finite or infinite) set of patterns $\tau_{1}, \tau_{2}, \ldots$, we denote by $\operatorname{Av}_{n}\left(\tau_{1}, \tau_{2}, \ldots\right.$ ) the set of permutations of size $n$ that avoid all the $\tau_{i}$ 's. Then the infinite union $\operatorname{Av}\left(\tau_{1}, \tau_{2}, \ldots\right)=\bigcup_{n} \operatorname{Av}_{n}\left(\tau_{1}, \tau_{2}, \ldots\right)$ is called a class of (pattern-avoiding) permutations. Equivalently, ${ }^{3}$ a class of permutations is a set $\mathcal{C}$ of permutations such that, for any $\sigma \in \mathcal{C}$ and any pattern $\pi$ of $\sigma$, it holds that $\pi \in \mathcal{C}$.

\footnotetext{
${ }^{3}$ This statement is folklore in the literature on permutation patterns. A proof can be found in [12], paragraph 5.1.2 for instance. See also [12], paragraph 7.2.3 for a proof that an infinite set of excluded patterns is sometimes necessary to describe a class.
} 
Classes of permutations have been intensively studied for their combinatorial and algorithmic properties over the last 50 years. An account of the past and current research on these classes can be found in $[12,28,40]$. Finding the enumeration of specific classes, defined by the avoidance of a small number of small patterns, has been one of the first problems studied in this field. It started with the proof that $\operatorname{Av}(\tau)$ is counted by the Catalan numbers, for any $\tau \in \mathfrak{S}_{3}$, and the research on this topic still continues, as witnessed by the summary [42]. The combinatorics of classes of permutations has however expanded in several other directions, including a general approach to the study of classes of permutations based on various notions of structure, like the substitution decomposition ([2], Proposition 2 or [40], Section 3.2) to which we shall return later in this introduction.

The probabilistic study of classes of permutations is much more recent and, just like their combinatorial study at its beginning, it focuses on the study of specific classes with small excluded patterns. More precisely, the probabilistic counterpart of the study of specific classes is centered on the following interesting question: Given a fixed pattern $\tau$, what can we say about a typical $\sigma$ in $\operatorname{Av}_{n}(\tau)$ (for large $n$ )? Recently, many authors have considered this problem for different choices of small patterns $\tau$. We mention a few of them:

- The question was initiated in a paper of Madras and Liu [32] in relation with a Monte-Carlo algorithm to approximate growth rates of permutation classes. In subsequent papers, Atapour and Madras [4] and Madras and Pehlivan [33] started the study of uniform permutations in $\operatorname{Av}_{n}(\tau)$ for small patterns $\tau$.

- In parallel, Miner and Pak [34] described very precisely the asymptotic shape of a uniform element in $\operatorname{Av}_{n}(\tau)$ for the 6 patterns $\tau$ in $\mathfrak{S}_{3}$. Dokos and Pak [16] have then obtained similar results for random doubly alternating Baxter permutations. Note also that Miner and Pak discuss at the end of their paper a possible connection with the Brownian excursion.

- Such a connection between $\operatorname{Av}_{n}(\tau)$ for $\tau \in \mathfrak{S}_{3}$ and the Brownian excursion is explained by Hoffman, Rizzolo and Slivken [21]. Many combinatorial consequences are given, in particular a precise description of fixed points of such permutations [20].

- In a parallel line of research, Bóna $[10,11]$ investigates the behaviour of $\mathbb{E}[\widetilde{\mathrm{occ}}(\pi, \sigma)]$ for $\sigma$ uniform in $\mathrm{Av}_{n}(132)$, and several fixed $\pi$ 's. Similar results for other permutation classes and various patterns $\pi$ have then been obtained by Homberger [22], Chang, Eu and Fu [14] and Rudolf [38].

- The question of finding limiting distributions for $\widetilde{\text { occ }}(\pi, \sigma)$ for $\sigma$ uniform in $\operatorname{Av}_{n}(\tau)$, rather than studying only its expectation, was raised by Janson, Nakamura and Zeilberger in [25]: the authors gave some algorithms to find limits of moments for small $\pi$ and $\tau$. A bit later, Janson [24] has given for every $\pi$ the asymptotic behaviour of the random variable $\widetilde{\mathrm{occ}}(\pi, \sigma)$ for $\sigma$ uniform in $\operatorname{Av}_{n}(132)$. For instance, he expresses in terms of the Brownian excursion area the asymptotic behaviour of $\widetilde{\mathrm{occ}}(12, \sigma)$. 
- In his recent thesis, Bevan describes the limit shape of permutations in so-called connected monotone grid classes [7], Chapter 6. This result is the first that deals with an infinite family of permutation classes.

- Even if it does not involve strictly speaking pattern-avoiding permutations, we mention the recent work of Kenyon, Kral', Radin and Winkler [27]. They prove a large deviation theorem for permutations seen as probability measures on the square. Their result yields limit shapes of random permutations with fixed densities of some fixed pattern $\pi_{1}, \ldots, \pi_{r}$. This parallels similar results on graphons, which are well-studied objects in random graph theory.

In the current paper, we are specifically interested in the class of separable permutations.

DEFINITION 1.1. A permutation $\sigma$ is separable if $\sigma$ avoids both 2413 and 3142 .

We obtain results similar to those of Janson [24] for Av(132), namely we study occurrences of any pattern $\pi$ in uniform random separable permutations. There is however an important difference between our work and all classes studied so far: random permutations in any of these previously studied classes have a limit, which is deterministic at first order, whereas random separable permutations have a limit, which is nondeterministic at first order. The limit of random separable permutations will be discussed in Section 1.3, and the proof that it is not deterministic will be given in Section 9.2. This is also visible on Figure 1, which shows two large typical separable permutations obtained using a Boltzmann random sampler.
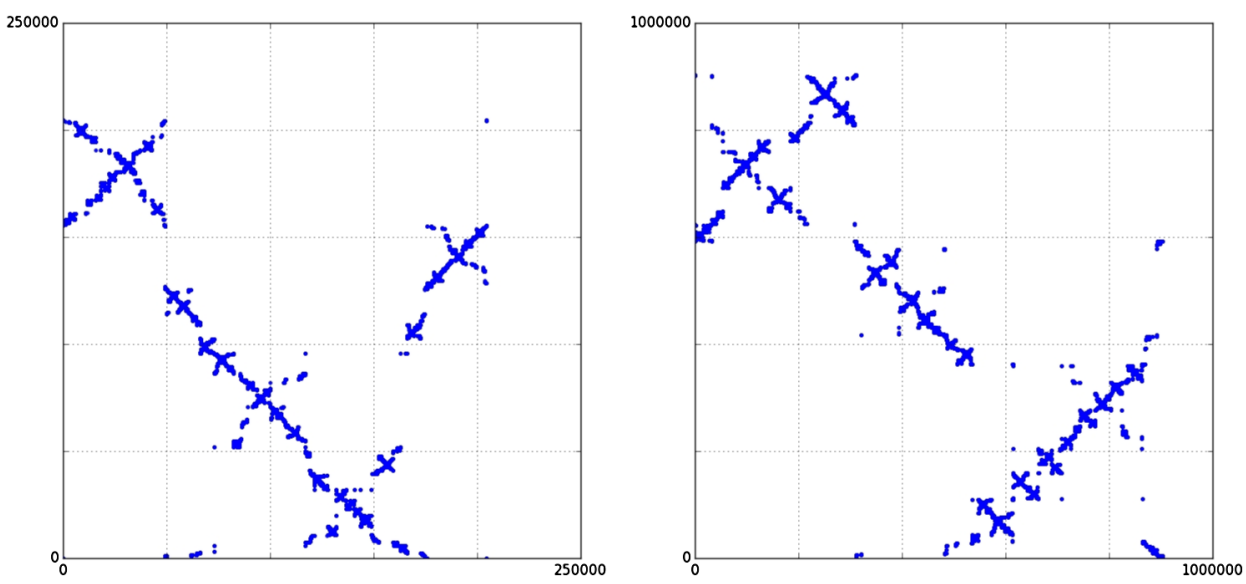

FIG. 1. Two uniform random separable permutations of sizes respectively $n=204,523$ and $n=903,073$ [ $a$ permutation $\sigma$ is represented here with its diagram: for every $i \leq n$, there is a dot at coordinates $\left.\left(i, \sigma_{i}\right)\right]$. 
There are several reasons that motivate our choice of studying the class of separable permutations, in addition to it being one of the most studied classes after $\operatorname{Av}(\tau)$ for $\tau$ of size 3. Separable permutations have a very nice and robust combinatorial structure: they can be completely decomposed using direct sums and skew sums and, therefore, can be represented as signed Schröder trees (see Section 2.2). This encoding with trees is essential in proving a variety of results about separable permutations in different fields, for instance:

- the algorithmic problem of PERMUtATIOn PATTERn MATCHING is NP-hard in general, but polynomial on separable permutations [13];

- from an enumerative combinatorics point of view, in addition to being simple to count, separable permutations display remarkable equipopularity properties, see [1].

Besides, separable permutations appear naturally in several problems, at first sight independent from permutation pattern theory:

- as the permutations sortable by certain sorting devices (pop-stacks in series) [5];

- as space-filling permutation matrices in bootstrap percolation [39];

- as possible polynomial interchanges (i.e., studying in which possible ways the relative order of the values of a family of polynomials can be modified when crossing a common zero) [18].

Finally, the class of separable permutations is the simplest case of a nontrivial substitution-closed (also called wreath-closed [2]) class, and we believe that the results obtained here might be extended to any substitution-closed class; see the discussion on universality in Section 1.4.

1.2. Overview of our results. Throughout this paper, let us denote by $\sigma_{n}$ a uniform separable permutation of size $n$. Our goal is to describe the limit of $\sigma_{n}$ when $n$ goes to infinity. Our main result gives, for any $\pi$, the asymptotics of the distribution of $\widetilde{\mathrm{occ}}\left(\pi, \sigma_{n}\right)$ when $n$ tends to infinity. We will see in Section 1.3 an equivalent formulation in terms of weak convergence of probability measures on the square.

Our main theorem is the following.

THEOREM 1.2. Let $\sigma_{n}$ be a uniform separable permutation of size $n$. There exists a collection of random variables $\left(\Lambda_{\pi}\right), \pi$ ranging over all permutations, defined on the same probability space, such that for all $\pi, 0 \leq \Lambda_{\pi} \leq 1$ and:

(i) If $\pi$ is a separable permutation of size at least $2, \Lambda_{\pi}$ is a nondeterministic random variable.

(If $\pi$ is the permutation of size $1, \Lambda_{\pi}=1$. If $\pi$ is not separable, $\Lambda_{\pi}=0$.)

(ii) For all $\pi$, when $n \rightarrow+\infty$,

$$
\widetilde{\operatorname{occ}}\left(\pi, \sigma_{n}\right) \stackrel{d}{\rightarrow} \Lambda_{\pi}
$$

where $\stackrel{d}{\rightarrow}$ denotes the convergence in distribution. 
(iii) Moreover, the convergence holds jointly, that is, for any finite sequence of permutations $\left(\pi_{1}, \ldots, \pi_{r}\right)$,

$$
\left(\widetilde{\mathrm{occ}}\left(\pi_{1}, \sigma_{n}\right), \ldots, \widetilde{\mathrm{occ}}\left(\pi_{r}, \sigma_{n}\right)\right) \stackrel{d}{\rightarrow}\left(\Lambda_{\pi_{1}}, \ldots, \Lambda_{\pi_{r}}\right)
$$

(On the right-hand side, the $\Lambda_{\pi_{i}}$ 's are not independent.)

Theorem 1.2 is not just an existential result: in this paper we give for any pattern $\pi$ a construction of $\Lambda_{\pi}$ (Definition 3.1, page 2155) that can be briefly explained as follows. There is a natural way (reviewed in Section 2.4) to extract a (signed) tree with $|\pi|$ leaves from a realization of the (signed) Brownian excursion. The variable $\Lambda_{\pi}$ is the probability that this tree is one of the separation trees of $\pi$ (see Section 2.2 for the definition).

Statement (i) of the theorem is proved in Section 9.2, while Statement (iii) [Statement (ii) being just a special case] is proved in Sections 4 to 7 , following the proof schema detailed in Section 3.4.

Theorem 1.2 shows in particular that, for every separable pattern $\pi$ of size $k$, the number of occurrences of $\pi$ in $\sigma_{n}$ must be renormalized by $n^{k}$ to have a nontrivial limit in distribution. This is in contrast with the result of Janson [24], Theorem 2.1 for $\sigma$ uniform in $\operatorname{Av}_{n}(132)$. Indeed, in his result, the numbers of occurrences of different patterns of the same size are normalized by different powers of $n$ to have nontrivial limits in distribution.

In addition to the convergence in distribution, we also prove the convergence of all joint moments (in fact, we first prove the convergence of joint moments, and then deduce the convergence in distribution). This is especially interesting since the joint moments in the limit can be computed explicitly.

More precisely, all joint moments can be expressed in the limit from expectations of $\Lambda_{\pi}$ 's (see Proposition 9.3), and the expectation of $\Lambda_{\pi}$ can be expressed in terms of the number $N_{\pi}$ of signed binary trees associated with the permutation $\pi$ (these are also sometimes called separation trees of $\pi$; see Section 2.2 for their definition). The latter is proved in Proposition 9.1, which reads as follows.

THEOREM 1.3. For any permutation $\pi$ of size $k$,

$$
\mathbb{E}\left[\widetilde{\operatorname{occ}}\left(\pi, \sigma_{n}\right)\right] \stackrel{n \rightarrow+\infty}{\longrightarrow} \frac{N_{\pi}}{2^{k-1} \mathrm{Cat}_{k-1}},
$$

where we denote by $\mathrm{Cat}_{k}:=\frac{1}{k+1}\left(\begin{array}{c}2 k \\ k\end{array}\right)$ the kth Catalan number.

REMARK 1.4. Our proof of Theorem 1.3 involves the random variables $\Lambda_{\pi}$, which are constructed from the Brownian excursion. We do not know if there exists a proof using only discrete arguments. 
In addition to being defined combinatorially, the numbers $N_{\pi}$ are easy to compute; see Observation 9.2. This gives, for example,

$$
\begin{aligned}
& \lim _{n \rightarrow+\infty} \mathbb{E}\left[\widetilde{\operatorname{occ}}\left(12, \sigma_{n}\right)\right]=\lim _{n \rightarrow+\infty} \mathbb{E}\left[\widetilde{\operatorname{occ}}\left(21, \sigma_{n}\right)\right]=\frac{1}{2} ; \\
& \lim _{n \rightarrow+\infty} \mathbb{E}\left[\widetilde{\operatorname{occ}}\left(123, \sigma_{n}\right)\right]=\lim _{n \rightarrow+\infty} \mathbb{E}\left[\widetilde{\operatorname{occ}}\left(321, \sigma_{n}\right)\right]=\frac{1}{4} ; \\
& \lim _{n \rightarrow+\infty} \mathbb{E}\left[\widetilde{\operatorname{occ}}\left(132, \sigma_{n}\right)\right]=\lim _{n \rightarrow+\infty} \mathbb{E}\left[\widetilde{\operatorname{occ}}\left(213, \sigma_{n}\right)\right]=\frac{1}{8} ; \\
& \lim _{n \rightarrow+\infty} \mathbb{E}\left[\widetilde{\operatorname{occ}}\left(231, \sigma_{n}\right)\right]=\lim _{n \rightarrow+\infty} \mathbb{E}\left[\widetilde{\operatorname{occ}}\left(312, \sigma_{n}\right)\right]=\frac{1}{8} .
\end{aligned}
$$

Limits of higher (joint) moments can also be computed explicitly, as shown in Proposition 9.3. For example, we obtain

$$
\begin{array}{r}
\lim _{n \rightarrow+\infty} \operatorname{Var}\left[\widetilde{\mathrm{occ}}\left(12, \sigma_{n}\right)\right]=\frac{1}{30} ; \\
\lim _{n \rightarrow+\infty} \operatorname{Var}\left[\widetilde{\mathrm{occ}}\left(132, \sigma_{n}\right)\right]=\frac{3}{560} \\
\lim _{n \rightarrow+\infty} \mathbb{E}\left[\widetilde{\mathrm{occ}}\left(12, \sigma_{n}\right) \cdot \widetilde{\mathrm{occ}}\left(123, \sigma_{n}\right)\right]=\frac{43}{280} .
\end{array}
$$

These values have been computed automatically with a Sage program [15] that the authors can make available on request. We refer to Section 9.1 for a discussion on the computation of joint moments, both from the theoretical and algorithmic points of view.

For the curious reader, we give the first few moments of $\Lambda_{12}$ [i.e., the limits of the first few moments of $\left.\widetilde{\text { occ }}\left(12, \sigma_{n}\right)\right]$ :

$$
\begin{aligned}
& \mathbb{E}\left[\Lambda_{12}\right]=\frac{1}{2} ; \quad \mathbb{E}\left[\Lambda_{12}^{2}\right]=\frac{17}{60} ; \quad \mathbb{E}\left[\Lambda_{12}^{3}\right]=\frac{7}{40} ; \\
& \mathbb{E}\left[\Lambda_{12}^{4}\right]=\frac{6361}{55,440} ; \quad \mathbb{E}\left[\Lambda_{12}^{5}\right]=\frac{1741}{22,176} .
\end{aligned}
$$

We did not recognize the first moments of any "usual" distribution on $[0,1]$.

1.3. Interpretation of our main result in terms of permutons. We recall the notion of permutons introduced in [23]. Note that, in [23], permutons are called limit permutations and have two equivalent definitions (see [23], Section 2.3); the name permuton was proposed in [19] in analogy with the graph analogue graphon.

DEFINITION 1.5. A permuton is a probability measure $\mu$ on the square $[0,1]^{2}$ with uniform marginals, that is, for any $a$ and $b$ with $0 \leq a \leq b \leq 1$,

$$
\mu([a, b] \times[0,1])=\mu([0,1] \times[a, b])=b-a .
$$



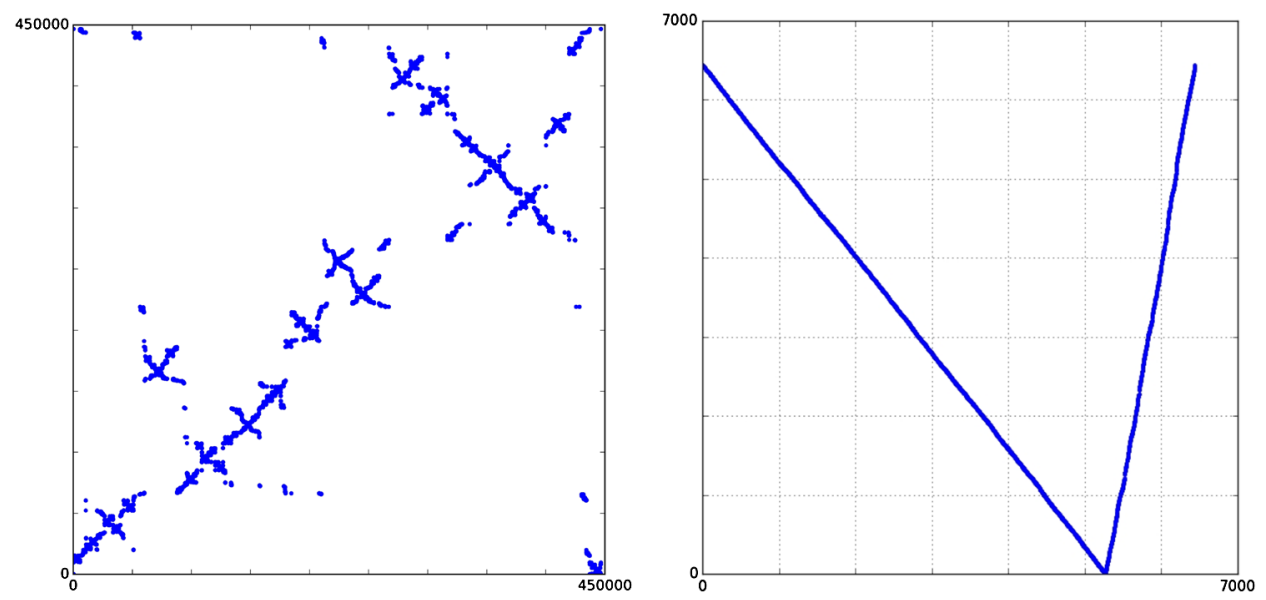

FIG. 2. On the left, a typical permutation of size 446,699 in the substitution-closed class whose simple permutations are 2413, 3142 and 24153. On the right, a typical permutation of size 6441 in the nonsubstitution-closed class $\operatorname{Av}(2413,1243,2341,531642,41352)$.

One can associate a permuton $\mu_{\sigma}$ to a permutation $\sigma$ of size $n$. We define $\mu_{\sigma}$ as having density $n$ on each square $[(i-1) / n, i / n] \times[(\sigma(i)-1) / n, \sigma(i) / n]$ (for $1 \leq i \leq n)$ and density 0 elsewhere; that is, each of these squares has total weight $1 / n$, uniformly distributed in it. This is a natural way to encode and rescale a permutation, very close to the graphical representation that we use on Figures 1 and 2.

Since permutons are measures, it is natural to speak about weak convergence of permutons. We will see in Section 8 that our main result, combined with previous results on permutons, implies the following.

THEOREM 1.6. Let $\sigma_{n}$ be a uniform random separable permutation of size $n$. There exists a random permuton $\boldsymbol{\mu}$ such that $\mu_{\sigma_{n}}$ tends to $\boldsymbol{\mu}$ in distribution in the weak convergence topology. Moreover, $\boldsymbol{\mu}$ is not deterministic.

The distribution of this permuton $\boldsymbol{\mu}$ is unique, since it is defined as a limit in distribution. The proof of the existence of $\boldsymbol{\mu}$ is not constructive, but involves the variables $\Lambda_{\pi}$, which are themselves built using a realization of the Brownian excursion. We therefore call $\boldsymbol{\mu}$ the Brownian separable permuton.

REMARK 1.7. There are many examples of convergence of large combinatorial structures towards continuum objects built from Brownian motion (or related processes: the Brownian bridge and excursion). One can mention:

- Random mappings and Brownian bridges;

- Random trees and Brownian trees;

- Random graphs and Brownian motion. 
A very good reference for these topics is [36]. Theorem 1.6 fits naturally in this body of literature.

Note that the fact that separable permutations have a Brownian limit in some sense should not come as a surprise. Indeed, separable permutations of size $n$ can be encoded by (signed) Schröder trees with $n$ leaves (see Section 2.2). Like for many families of trees, the limit of Schröder trees with a fixed number of leaves (leaving signs aside) is related to the Brownian excursion: more precisely, Pitman and Rizzolo [37] and Kortchemski [29] proved that the contour of a uniform Schröder tree with $n$ leaves tends to the Brownian excursion. This result is essential in our approach.

1.4. Perspectives. We think that the Brownian separable permuton $\boldsymbol{\mu}$ is an interesting object and is worth being studied. In particular, we would like to address the following questions.

Construction of $\boldsymbol{\mu}$ At the moment, $\boldsymbol{\mu}$ is defined in a indirect way, as limit of discrete objects. Is there a way to define/construct the random measure $\boldsymbol{\mu}$ directly in the continuum, for example, as a function of the (signed) Brownian excursion?

Properties of $\boldsymbol{\mu}$ It would be interesting to find some almost-sure properties of $\boldsymbol{\mu}$. Is it absolutely continuous or singular with respect to Lebesgue measure on the square? (Note that, since its marginals are uniform, it cannot have atoms.) One also expects that $\boldsymbol{\mu}$ is fractal in some sense, because of the link with the Brownian excursion and the visual aspect of the simulations in Figure 1. This raises the following question: what is the Hausdorff dimension of its support?

Universality of $\boldsymbol{\mu}$ We believe that $\boldsymbol{\mu}$ is the limit (in the sense of permutons) of many other classes of random pattern-avoiding permutations. Recall indeed that the class of separable permutations is the first nontrivial case of a substitutionclosed class of permutations. Such classes are those whose structure is well understood using the substitution decomposition mentioned earlier in this introduction, even more so when they contain a finite number of so-called simple permutations (see [40], Section 3.2). For any substitution-closed class, the permutations it contains may be represented by trees, called (substitution) decomposition trees. These generalize signed Schröder trees by introducing other types of vertices, labeled by the simple permutations in the class. As the encoding of separable permutations by Schröder trees is crucial in this work, our results might extend to all substitution-closed classes containing finitely many simple permutations. Figure 2 shows two typical permutations: one in a substitution-closed class, one in a class that is not closed under substitution. On these examples, it seems clear that the first one looks similar to the separable case, whereas the second one does not.

Note added after the peer-review process: after the submission of the present article, subsequent results have been achieved on the topic. A construction of the 
Brownian separable permuton has been given by Maazoun in [31]. One can prove from this construction that the support of $\boldsymbol{\mu}$ is completely disconnected and has Hausdorff dimension 1, with probability 1 . Moreover, the authors of this paper, together with Maazoun, have proved the following universality result: under a simple sufficient condition, a uniform random permutation in a substitution-closed class does indeed converge towards the Brownian separable permuton (or a trivial oneparameter deformation of it); see [6].

\subsection{Outline of the paper. Our paper is organized as follows:}

- Section 2 gives all the preliminaries needed to define the limit random variables $\Lambda_{\pi}$ and records a lot of easy useful facts about permutations and trees.

- Section 3 defines the variables $\Lambda_{\pi}$ and presents the structure of the proof of Theorem 1.2(iii).

- Sections 4 to 6 go through the several steps of this proof (the outline of the proof itself is given in Section 3.4).

- We gather all the arguments and conclude the proof of Theorem 1.2(iii) in Section 7.

- Section 8 contains the proof of the permuton interpretation of our main result: Theorem 1.6.

- Section 9 studies some properties of $\Lambda_{\pi}$ : combinatorial formulas for the moments in Section 9.1 and proof of Theorem 1.2(i) $\left(\Lambda_{\pi}\right.$ is not deterministic) in Section 9.2.

- We collect several useful properties of the Brownian excursion in the Appendix.

\section{Permutations, trees and excursions.}

\subsection{Basics on trees.}

DEFINITION 2.1. A Schröder tree is either a leaf, or consists of a root vertex with an ordered $k$-tuple of subtrees attached to the root $(k \geq 2)$, which are themselves Schröder trees.

Nonleaf vertices of a tree are called internal vertices.

We consider only finite Schröder trees, that is, those having finitely many leaves and internal vertices.

In our context, the size of a tree $t$ is its number of leaves. It is denoted $|t|$, whereas $\# t$ denotes the number of vertices of $t$ (including both leaves and internal vertices).

Because every internal vertex of a Schröder tree has at least 2 children, it follows immediately (by induction) the following.

OBSERVATION 2.2. For every Schröder tree $t, 2|t| \geq \# t+1$. 
A binary tree is a Schröder tree where there are exactly 2 subtrees attached to every internal vertex.

In this article, we consider unlabeled trees. Nevertheless, since we work with plane trees (i.e., trees in which the subtrees attached to a vertex are ordered), there is a canonical way to label the leaves of a tree (from left to right). Then a subset of the set of leaves of a tree $t$ is canonically represented by a subset $I$ of $[|t|]$.

DEFINITION 2.3. Let $t$ be a Schröder tree and $u$ and $v$ be two vertices of $t$. Denote by $r$ the root of $t$. The (first) common ancestor of $u$ and $v$ is the vertex furthest away from $r$ that appears on both paths from $r$ to $u$ and from $r$ to $v$ in $t$.

Definition 2.4. Let $t$ be a Schröder tree. Any subset $I$ of the leaves of $t$ induces a subtree $t_{I}$ of $t$, which is also a Schröder tree, defined as follows:

- the leaves of $t_{I}$ are the elements of $I$;

- the internal vertices of $t_{I}$ are the vertices of $t$ that are common ancestors of two leaves in $I$;

- the ancestor-descendant relation in $t_{I}$ is inherited from the one in $t$;

- the order between the children of an internal vertex of $t_{I}$ is inherited from $t$.

Note that if $t$ is a binary tree, then so is $t_{I}$.

DEFINITION 2.5. A signed Schröder tree is a pair $(t, \varepsilon)$, where $t$ is a Schröder tree and $\varepsilon$ a function from the set of internal vertices of $t$ to $\{+,-\}$.

Definition 2.6. Let $(t, \varepsilon)$ be a signed Schröder tree. Any subset $I$ of the leaves of $t$ induces a signed subtree $\left(t_{I}, \varepsilon_{I}\right)$ of $(t, \varepsilon)$, where $t_{I}$ is as in Definition 2.4 and $\varepsilon_{I}$ is the restriction of $\varepsilon$ on the set of internal vertices of $t_{I}$.

EXAMPLE 2.7. Consider the signed Schröder tree on the left-hand side of Figure 3. To ease the presentation, we have indicated the canonical labeling of its leaves from left to right. We take the subset of leaves $I=\{2,4,7\}$ (circled on the picture). Then the signed subtree $\left(t_{I}, \varepsilon_{I}\right)$ is represented on the right-hand side of Figure 3. (Again labels on the leaves are here to simplify the presentation, our objects are in essence not labeled.)

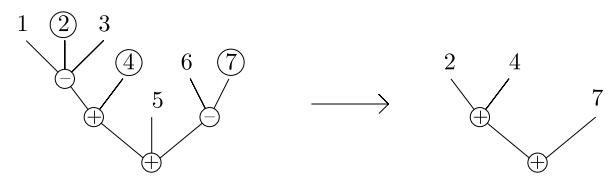

FIG. 3. Subtree of a signed Schröder tree. The labels on the leaves are here only to witness the embedding of the right tree in the left one. 


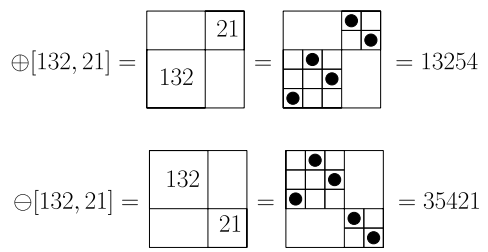

FIG. 4. Direct sum and skew sum of permutations on their diagrams. Recall that the diagram of a permutation $\sigma$ of size $n$ is the set of points at coordinates $\left(i, \sigma_{i}\right)$, for $1 \leq i \leq n$.

\subsection{Separable permutations and Schröder trees.}

DEFINITION 2.8. Let $\pi$ and $\sigma$ be two permutations of respective sizes $k$ and $\ell$. Their direct sum and skew sum are the permutations of size $k+\ell$ defined by

$$
\begin{aligned}
& \oplus[\pi, \sigma]=\pi \oplus \sigma=\pi_{1} \cdots \pi_{k}\left(\sigma_{1}+k\right) \cdots\left(\sigma_{\ell}+k\right) \quad \text { and } \\
& \ominus[\pi, \sigma]=\pi \ominus \sigma=\left(\pi_{1}+\ell\right) \cdots\left(\pi_{k}+\ell\right) \sigma_{1} \cdots \sigma_{\ell} .
\end{aligned}
$$

The operators $\oplus$ and $\ominus$ being associative, direct sums and skew sums with $r \geq 2$ components are defined in the obvious way. We will use the notation $\oplus\left[\pi^{1}, \ldots, \pi^{r}\right]$ instead of $\pi^{1} \oplus \cdots \oplus \pi^{r}$ (and similarly for $\ominus$ ). Examples with $k=3, \ell=2$ are provided in Figure 4, which also illustrates the graphical interpretation of these operations on permutation diagrams.

Definition 2.9. Let $(t, \varepsilon)$ be a signed Schröder tree. The permutation associated with $(t, \varepsilon)$, denoted perm $(t, \varepsilon)$, is inductively defined by:

- if $t$ is a leaf, then $\operatorname{perm}(t, \varepsilon)=1$;

- otherwise, denoting by $t_{1}, \ldots, t_{r}$ the children of the root of $t$ from left to right, and $\varepsilon_{i}$ the restriction of $\varepsilon$ to the vertices of $t_{i}$,

$$
\operatorname{perm}(t, \varepsilon)= \begin{cases}\oplus\left[\operatorname{perm}\left(t_{1}, \varepsilon_{1}\right), \ldots, \operatorname{perm}\left(t_{r}, \varepsilon_{r}\right)\right] & \text { if the root of } t \text { has sign }+; \\ \ominus\left[\operatorname{perm}\left(t_{1}, \varepsilon_{1}\right), \ldots, \operatorname{perm}\left(t_{r}, \varepsilon_{r}\right)\right] & \text { if the root of } t \text { has sign }-\end{cases}
$$

If $\operatorname{perm}(t, \varepsilon)=\sigma$, we say that $(t, \varepsilon)$ is a signed tree of $\sigma$.

Note that the $i$ th leaf of $(t, \varepsilon)$ (from left to right) corresponds to the $i$ th element of $\operatorname{perm}(t, \varepsilon)$. Binary signed trees of a permutation $\sigma$ are sometimes called separation trees of $\sigma$.

For example, let $(t, \varepsilon)$ be the tree on the left-hand side of Figure 3 of Example 2.7. Then

$$
\begin{aligned}
\operatorname{perm}(t, \varepsilon) & =\oplus[\oplus[\ominus[1,1,1], 1], 1, \ominus[1,1]]=\oplus[\oplus[321,1], 1,21] \\
& =\oplus[3214,1,21]=3214576 .
\end{aligned}
$$


On the other hand, if we consider the tree $\left(t_{I}, \varepsilon_{I}\right)$ on the right-hand side of Figure 3, we have

$$
\operatorname{perm}\left(t_{I}, \varepsilon_{I}\right)=\oplus[\oplus[1,1], 1]=123 .
$$

In other words, $(t, \varepsilon)$ and $\left(t_{I}, \varepsilon_{I}\right)$ are signed trees of 3214576 and 123 , respectively.

OBSERVATION 2.10. There is an important consequence of the definition of the signed tree $(t, \varepsilon)$ of a permutation $\sigma$, which is easily seen on our figures. For $i<j$, then $\sigma_{i}<\sigma_{j}$ (resp., $\sigma_{i}>\sigma_{j}$ ) if and only if the common ancestor of leaves $i, j$ has sign + (resp., - ).

A consequence of this observation is that the map perm is compatible with taking substructures.

OBSERVATION 2.11. Let $(t, \varepsilon)$ be a signed Schröder tree. Let $I$ be a subset of $[|t|]$ and $\left(t_{I}, \varepsilon_{I}\right)$ be the signed subtree of $(t, \varepsilon)$ induced by $I$. Then it holds that $\operatorname{pat}_{I}(\operatorname{perm}(t, \varepsilon))=\operatorname{perm}\left(t_{I}, \varepsilon_{I}\right)$.

We continue Example 2.7. Taking $I=\{2,4,7\}$, we have seen in previous examples that $\operatorname{perm}(t, \varepsilon)=3214576$ and $\operatorname{perm}\left(t_{I}, \varepsilon_{I}\right)=123$. According to Observation 2.11 , we should have pat ${ }_{\{2,4,7\}}(3214576)=123$, which is indeed the case. This is illustrated by Figure 5 .

In the Introduction, separable permutations have been defined as avoiding specific patterns. In fact, they can also been characterized using signed Schröder trees.

PROPOSITION 2.12. Separable permutations are exactly those that can be obtained as $\operatorname{perm}(t, \varepsilon)$ for a signed binary tree $(t, \varepsilon)$, or equivalently, those that can be obtained as perm $(t, \varepsilon)$ for a signed Schröder tree $(t, \varepsilon)$.

PROOF. The first part (with binary trees) has been established in [13].

Assume now that a permutation $\sigma$ can be obtained as $\operatorname{perm}(t, \varepsilon)$ for a signed Schröder tree $(t, \varepsilon)$; we need to prove that $\sigma$ can also be obtained as perm $\left(t^{\prime}, \varepsilon^{\prime}\right)$ for a signed binary tree $\left(t^{\prime}, \varepsilon^{\prime}\right)$. To obtain $\left(t^{\prime}, \varepsilon^{\prime}\right)$ from $(t, \varepsilon)$, it is enough to replace every vertex with a label $\delta$ and $k>2$ subtrees $t_{1}, \ldots, t_{k}$ by a binary tree with $k$ leaves on which $t_{1}, \ldots, t_{k}$ are pending and with all internal vertices labeled $\delta$.

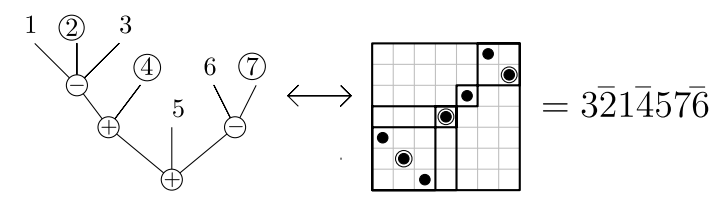

FIG. 5. One of the signed trees associated with $\sigma=3214576$. The elements of the pattern $\operatorname{pat}_{\{2,4,7\}}(\sigma)$ are circled/overlined. 


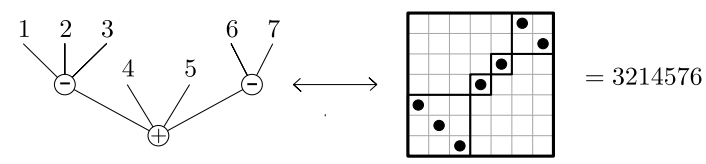

FIG. 6. The decomposition tree of a separable permutation.

Figure 12 (page 2180) shows all binary trees that can be obtained by the above construction from a specific signed Schröder tree.

The advantage of Schröder trees over binary trees is that the correspondence tree-permutation can be made one-to-one in more natural way.

PROPOSITION 2.13. The map perm is a size-preserving bijection between separable permutations and signed Schröder trees in which the signs alternate [i.e., all internal vertices at even (resp., odd) distance from the root have the same sign as the root (resp., the opposite sign)].

Proposition 2.13 is a consequence of the more general substitution decomposition theorem for permutations [2], Proposition 2. In this context, the unique signed Schröder tree $(t, \varepsilon)$ in which the signs alternate such that $\operatorname{perm}(t, \varepsilon)=\pi$ is called the (substitution) decomposition tree of $\pi$.

Let us return to Example 2.7. The permutation 3214576 which is induced by the tree on the left-hand side of Figure 3 is separable and the Schröder tree in which signs alternate that corresponds to it is shown in Figure 6.

Recall that $\sigma_{n}$ denotes a uniform random separable permutation of size $n$. Also, throughout the paper, we denote by $T_{n}$ a uniform random Schröder tree with $n$ leaves. From Proposition 2.13, we have the following.

COROLlary 2.14. Let $B$ be a balanced Bernoulli variable with values in $\{+,-\}$, independent from $T_{n}$. Let $\mathcal{E}_{n}$ be the sign function on the internal vertices of $T_{n}$, such that the signs alternate and the root of $T_{n}$ has sign $B$. Then $\sigma_{n}$ has the same distribution as $\operatorname{perm}\left(T_{n}, \mathcal{E}_{n}\right)$.

Pitman and Rizzolo observed (see [37], Theorem 1) that $T_{n}$ behaves like a Galton-Watson tree conditioned to have $n$ leaves (we refer to [41], Section 3 for basics of Galton-Watson trees).

PROPOSITION 2.15. Let $v$ be the probability distribution defined by

$$
v(0)=2-\sqrt{2}, \quad v(1)=0, \quad v(i)=\left(1-\frac{\sqrt{2}}{2}\right)^{i-1} \quad(\text { for all } i \geq 2) .
$$

Then:

- $v$ has mean 1 and variance $4(\sqrt{2}-1)$; 
- $T_{n}$ has the same distribution as a Galton-Watson tree with offspring distribution $v$, conditioned to have $n$ leaves.

Note that there are actually infinitely many probability distributions $v$ such that $T_{n}$ has the same distribution as a Galton-Watson tree with offspring distribution $v$, conditioned to have $n$ leaves. The one chosen in Proposition 2.15 is such that $v$ has mean 1. The Galton-Watson tree model is then critical, and most convergence results in the literature are established in this case (see, e.g., Propositions 3.5 and 2.23 below).

\subsection{Contours and excursions.}

DEFinition 2.16. An excursion is a continuous function $f:[0,1] \rightarrow$ $[0,+\infty)$ with $f(0)=f(1)=0$.

Note that with our convention we allow $f(t)=0$ for $t \notin\{0,1\}$. We associate canonically with a tree an excursion, called its contour.

DEFinition 2.17 (Contour $C_{t}$ ). Let $t$ be a (binary or Schröder) tree. Recall that \#t denotes the total number of vertices of $t$, and denote by $V$ the set of vertices of $t$.

Consider the function $\mathrm{dfs}_{t}:\{0,1, \ldots, 2 \# t-2\} \rightarrow V$ (dfs stands for depth first search) defined by:

- $\operatorname{dfs}_{t}(0)$ is the root of $t$;

- if $\operatorname{dfs}_{t}(i)=v$, then $\operatorname{dfs}_{t}(i+1)$ is the leftmost child of $v$ that has not yet been visited, if it exists, and the parent of $v$ otherwise.

The contour of $t$ is the function $C_{t}:[0,2 \# t-2] \rightarrow[0 ;+\infty)$ such that for all integers $i$ in $[0,2 \# t-2], C_{t}(i)$ is the distance from the root of $t$ to the vertex $\mathrm{dfs}_{t}(i)$, and $C_{t}$ is linear between those points.

EXAMPLE 2.18. An example of a tree $t$ and its contour $C_{t}$ is given on Figure 7 (the reader should disregard the signs for the moment). The tiny numbers beside each vertex $v$ indicate the times $i$ in $[0,2 \# t-2]$ such that $\operatorname{dfs}_{t}(i)=v$.

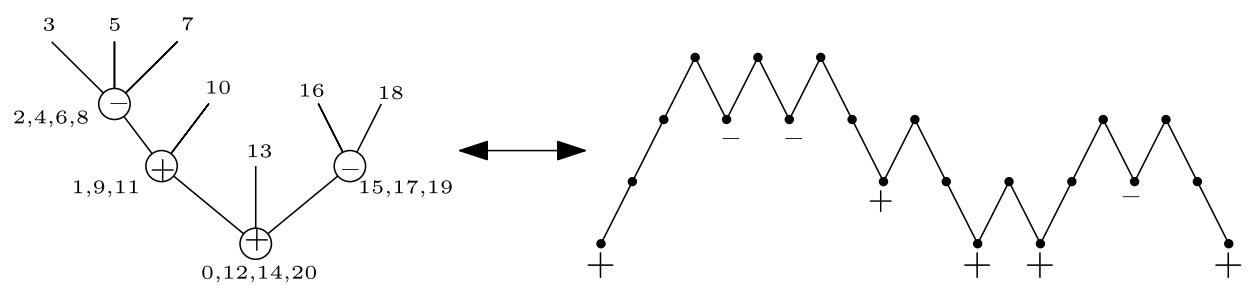

FIG. 7. A (signed) tree and its (signed) contour. 
DEFINITION 2.19 (Normalized contour $\widetilde{C_{t}}$ ). Let $t$ be a Schröder tree. The normalized contour of $t$ is the function $\widetilde{C}_{t}:[0,1] \rightarrow[0,+\infty)$ defined as follows:

$$
\text { for all } u \in[0,1], \quad \widetilde{C_{t}}(u)=\frac{1}{\sqrt{|t|}} C_{t}((2 \# t-2) u) \text {. }
$$

By definition, both $C_{t}$ and $\widetilde{C_{t}}$ are continuous, so that $\widetilde{C_{t}}$ is an excursion.

Some properties of the local maxima and local minima of $\widetilde{C_{t}}$ follow from its definition, and will be essential for our purpose.

OBSERVATION 2.20. Let $t$ be a tree with $|t|>1$. If there is a local maximum of $\widetilde{C}_{t}$ at $u$, then $u=\frac{i}{(2 \# t-2)}$ for some integer $i$ and $\operatorname{dfs}_{t}(i)$ is a leaf of $t$. This defines a bijection between the leaves of $t$ and the local maxima of $\widetilde{C_{t}}$.

This allows us to identify the leaves of $t$ with the $x$-coordinates of the local maxima of $\widetilde{C_{t}}$. We will often do so in the sequel, and we introduce the following notation.

DEFINITION $2.21\left(\ell_{t}\right)$. For any tree $t$ with $|t|>1$, we define $\ell_{1}<\cdots<\ell_{|t|}$ to be the $x$-coordinates of the local maxima of $\widetilde{C}_{t}$ (which correspond to the leaves of $t)$, and we set $\ell_{t}=\left\{\ell_{1}, \ldots, \ell_{|t|}\right\} \subset[0,1]$.

OBSERVATION 2.22. Let $t$ be a tree with $|t|>1$. If there is a local minimum of $\widetilde{C}_{t}$ in $u$, then $u=\frac{i}{(2 \# t-2)}$ for some integer $i$ and $\operatorname{dfs}_{t}(i)$ is an internal vertex of $t$. Note however that a single internal vertex can correspond to several local minima of $\widetilde{C}_{t}$.

Using the description of Schröder trees as Galton-Watson trees (see Proposition 2.15), Pitman and Rizzolo [37] and Kortchemski [29] have proved that the normalized contour of a uniform Schröder tree converges in distribution to a multiple of the Brownian excursion. ${ }^{4}$ Throughout the paper, we denote by $e$ the Brownian excursion.

Proposition 2.23. The following convergence holds in distribution in the space $\mathcal{C}[0,1]$ of real-valued continuous functions on $[0,1]$ :

$$
\left(\widetilde{C_{T_{n}}}(u)\right)_{u \in[0,1]} \stackrel{n \rightarrow+\infty}{\longrightarrow}(\lambda \cdot e(u))_{u \in[0,1]}
$$

where $\lambda=\sqrt{2+3 / \sqrt{2}}$.

\footnotetext{
${ }^{4}$ For the readers who need background on the Brownian excursion, we have collected in the Appendix (one of) its definition(s), together with some useful properties, and bibliographical references.
} 
We now define signed analogues of contours and excursions.

DEFINITION 2.24. A signed excursion is a pair $(f, s)$ where $f$ is an excursion and $s$ a function from the set of the local minima of $f$ to $\{+,-\}$.

From Observation 2.22, we may define the signed contour of a signed tree as follows.

DEFINITION 2.25. Let $(t, \varepsilon)$ be a signed Schröder tree. The signed contour of $(t, \varepsilon)$ is the pair $\left(\widetilde{C}_{t}, s\right)$, where $s$ associates to every local minimum of $\widetilde{C}_{t}$ reached in $u=\frac{i}{(2 \# t-2)}$ the sign of the internal vertex $\operatorname{dfs}_{t}(i)$ of $t$.

EXAMPLE 2.26. The reader is now invited to look again at Figure 7, taking the signs into consideration.

We may also define the signed Brownian excursion by the following.

DEFINITION 2.27. The signed Brownian excursion is the pair $(e, S)$ where $e$ is the Brownian excursion and $S$ is the function assigning balanced independent signs on the local minima of $e$.

The probability space and the $\sigma$-algebra on which this object is constructed will be introduced in Section 2.5.

REMARK 2.28. Some readers may have seen the term "signed Brownian excursion" refer to an excursion of Brownian motion whose length has been normalized to 1 , but that may be either positive on $(0,1)$ or negative on $(0,1)$. The meaning of this expression in our paper is different.

REMARK 2.29. Consider a uniform random Schröder tree $T_{n}$ of size $n$ and a random sign function $\mathcal{E}_{n}$ on its internal vertices defined as in Corollary 2.14. If we use Definition 2.25 on $\left(T_{n}, \mathcal{E}_{n}\right)$, we get a random signed excursion $\left(\widetilde{C_{T_{n}}}, S_{n}\right)$. Since $\widetilde{C_{T_{n}}}$ converges towards a multiple of the Brownian excursion $e$, a natural question is the convergence of $\left(\widetilde{C_{T_{n}}}, S_{n}\right)$ towards the signed Brownian excursion. A first difficulty in proving such a result would be to find a good topology on signed excursions. Another, maybe deeper, obstacle is that the signs assigned to the local minima in $\left(\widetilde{C_{T_{n}}}, S_{n}\right)$ are far from independent: recall that on the corresponding tree, signs on internal vertices alternate. Our work shows the convergence of $\left(\widetilde{C_{T_{n}}}, S_{n}\right)$ to $(e, S)$ in a weaker sense. Indeed, the next subsection explains how to extract a permutation of any fixed size from a signed excursion, and Theorem 1.2(iii) proves the joint convergence in distribution of the permutations extracted from $\left(\widetilde{C_{T_{n}}}, S_{n}\right)$ to those extracted from $(e, S)$. 
2.4. Extracting trees and permutations from excursions. Given $k$ points in an excursion, there is a natural way to associate a tree with this data. We now explain this construction. This is classical in the random tree literature and can, for example, be found in Le Gall's survey [30], Section 2.5 (except that he considers geometric trees, that is, with edge-lengths, while we are only interested in combinatorial trees).

DEFinition 2.30 (Tree). Let $f$ be an excursion and $\mathbf{x}=\left\{x_{1}, \ldots, x_{k}\right\}$ be a set of $k$ points in $[0,1]$. Without loss of generality, we assume $x_{1}<\cdots<x_{k}$. For all $1 \leq i \leq k-1$, let $m_{i}$ be the minimum value of $f$ on $\left[x_{i}, x_{i+1}\right]$.

We associate to $(f, \mathbf{x})$ a Schröder tree Tree $(f, \mathbf{x})$, defined recursively as follows. If $k=1$, then Tree $(f, \mathbf{x})$ is a leaf.

Otherwise, let $m=\min _{i}\left\{m_{i}\right\}$ and denote by $i_{1}<i_{2}<\cdots<i_{p}$ all the indices $i_{j}$ such that $m_{i_{j}}=m$. For notational convention, let $i_{0}=0$ and $i_{p+1}=k$. For all $0 \leq j \leq p$, define $\mathbf{x}^{(j)}=\left\{x_{i_{j}+1}, \ldots, x_{i_{j+1}}\right\}$. Then Tree $(f, \mathbf{x})$ is the tree whose root has arity $p+1$ and whose children are $\operatorname{Tree}\left(f, \mathbf{x}^{(0)}\right), \ldots, \operatorname{Tree}\left(f, \mathbf{x}^{(p)}\right)$.

ObSERVATION 2.31. If all $m_{i}$ are distinct, then $\operatorname{Tree}(f, \mathbf{x})$ is a binary tree.

EXAMPLE 2.32. An example of the construction presented in Definition 2.30 is given on Figure 8 (the reader should disregard the signs for the moment).

ObSERVATion 2.33. By construction, the leaves of Tree $(f, \mathbf{x})$ are in one-toone correspondence with the $x_{i}$ 's. Moreover, to each $m_{i}$ corresponds an internal vertex of Tree $(f, \mathbf{x})$; note however that several $m_{i}$ 's may correspond to the same vertex of Tree $(f, \mathbf{x})$ (this happens exactly for $m_{i}$ and $m_{j}$ having the same value such that no $m_{k}$ for $i<k<j$ is smaller). In addition, the common ancestor of the leaves corresponding to $x_{i}$ and $x_{i+1}$ in Tree $(f, \mathbf{x})$ is the internal vertex that corresponds to $m_{i}$. More generally, the common ancestor of the leaves corresponding to $x_{i}$ and $x_{j}$, with $i<j$, is the internal vertex that corresponds to $\min _{i \leq h<j}\left\{m_{h}\right\}$.

An interesting special case consists in considering the normalized contour $\widetilde{C_{t}}$ of a tree $t$, and choosing $\mathbf{x}$ to be a subset of $\boldsymbol{\ell}_{t}$ (defined in Definition 2.21).

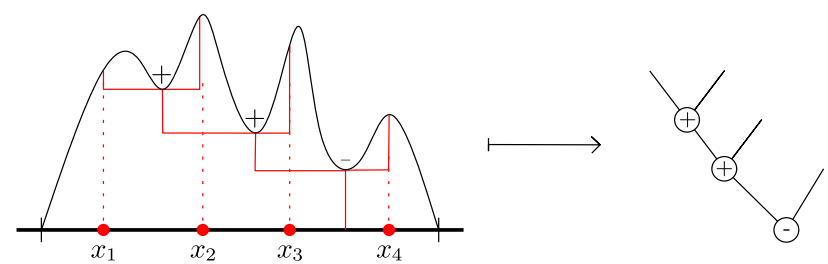

FIG. 8. Extracting a (signed) tree from a (signed) excursion. 
OBSERVATION 2.34. Let $t$ be a Schröder tree, and $I$ be a subset of its set of leaves. Let $\mathbf{x}$ be the subset of $\boldsymbol{\ell}_{t}$ corresponding to the $x$-coordinates of these leaves. Then Tree $\left(\widetilde{C_{t}}, \mathbf{x}\right)=t_{I}$, that is to say the tree extracted from $\mathbf{x}$ in the contour of $t$ is the same as the subtree of $t$ induced by $I$.

A similar statement holds when extracting trees in excursions that are not necessarily contours; this observation (or rather its signed analogue) will be useful in Section 4.

OBSERVATION 2.35. Let $f$ be an excursion and $\mathbf{x}=\left\{x_{1}, \ldots, x_{k}\right\}$ be a set of $k$ points in $[0,1]$. Let $t=\operatorname{Tree}(f, \mathbf{x})$. Let $\mathbf{y}$ be a subset of $\mathbf{x}$, and $I$ be the corresponding subset of the set of leaves of $t$. Then the tree extracted from $\mathbf{y}$ in $f$ is the substree of $t$ induced by $I$, that is, $\operatorname{Tree}(f, \mathbf{y})=t_{I}$.

We now discuss the signed analogue of the extraction of trees from excursions.

DEFINITION 2.36 ( Tree $\left._{ \pm}\right)$. Let $(f, s)$ be a signed excursion, and let also $\mathbf{x}=$ $\left\{x_{1}, \ldots, x_{k}\right\}$ be a set of $k$ points in [0,1], with $x_{1}<\cdots<x_{k}$. As in Definition 2.30, for all $1 \leq i \leq k-1$, denote by $m_{i}$ the minimum value of $f$ on $\left[x_{i}, x_{i+1}\right]$.

We assume that the following condition holds:

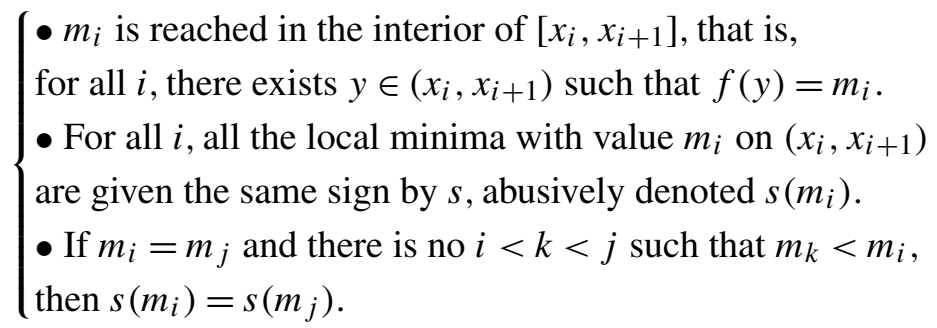

Then $\operatorname{Tree}_{ \pm}(f, s, \mathbf{x})$ is defined like Tree $(f, \mathbf{x})$, except that, at each stage of the construction, we associate with the root the sign of the corresponding local minimum (or minima) of $f$. Doing so, at the end of the construction, every internal vertex of Tree $_{ \pm}(f, s, \mathbf{x})$ has a sign.

Condition $(\mathcal{C})$ ensures that the signs are well defined. Indeed, the first condition guarantees that $f$ has a minimum on $\left(x_{i}, x_{i+1}\right)$, which is therefore a local minimum of $f$ (of value $m_{i}$ ), and consequently has a sign. The second condition implies that, for all $i$, all minima of $f$ on $\left(x_{i}, x_{i+1}\right)$ have the same sign, and the third one ensures that (with the notation of Definition 2.30) all $m_{i_{j}}$ have the same sign, and recursively so.

Because a separable permutation is associated with each signed Schröder tree (see Definition 2.9 and Proposition 2.12), we can extract a separable permutation from a signed excursion in which $k$ points are chosen. 
Definition 2.37. Let $(f, s)$ be a signed excursion and $\mathbf{x}=\left\{x_{1}, \ldots, x_{k}\right\}$ be a set of $k$ points in $[0,1]$. Assume $(f, s, \mathbf{x})$ satisfies Condition $(\mathcal{C})$. The separable permutation associated with $(f, s, \mathbf{x})$ is

$$
\operatorname{Perm}(f, s, \mathbf{x}):=\operatorname{perm}\left(\operatorname{Tree}_{ \pm}(f, s, \mathbf{x})\right) .
$$

EXAMPLE 2.38. The reader is now invited to look again at Figure 8, taking the signs into consideration. The permutation associated to the signed tree on the right is 2341, so that in this case, $\operatorname{Perm}(f, s, \mathbf{x})=2341$.

OBSERVATION 2.39. When considering a signed tree $(t, \varepsilon)$ and its signed contour $\left(\widetilde{C_{t}}, s\right)$, and choosing $\mathbf{x}$ as a subset of $\ell_{t}$, then Condition $(\mathcal{C})$ is always satisfied. Indeed, all local minima of $\widetilde{C_{t}}$ that are required to have the same sign correspond to the same internal vertex of $t$.

Moreover, we have the signed equivalents of Observations 2.34 and 2.35.

OBSERVATION 2.40. Let $(t, \varepsilon)$ be a signed Schröder tree and $\left(\widetilde{C_{t}}, s\right)$ be its signed contour. Let $I$ be a subset of the set of leaves of $t$, and $\mathbf{x}$ be the corresponding subset of $\ell_{t}$. Then Tree ${ }_{ \pm}\left(\widetilde{C}_{t}, s, \mathbf{x}\right)=\left(t_{I}, \varepsilon_{I}\right)$, that is, the signed tree extracted from $\mathbf{x}$ in the signed contour of $(t, \varepsilon)$ is defined and is the same as the signed subtree of $(t, \varepsilon)$ induced by $I$.

OBSERVATION 2.41. Let $(f, s)$ be a signed excursion and $\mathbf{x}=\left\{x_{1}, \ldots, x_{k}\right\}$ be a set of $k$ points in $[0,1]$. Assume that $\operatorname{Tree}_{ \pm}(f, s, \mathbf{x})$ is defined and let us denote $(t, \varepsilon)=$ Tree $_{ \pm}(f, s, \mathbf{x})$. Let $\mathbf{y}$ be a subset of $\mathbf{x}$, and let $I$ be the corresponding subset of the set of leaves of $(t, \varepsilon)$. Then the signed tree extracted from $\mathbf{y}$ in $(f, s)$ is defined and is the substree of $(t, \varepsilon)$ induced by $I$, that is, Tree ${ }_{ \pm}(f, s, \mathbf{y})=\left(t_{I}, \varepsilon_{I}\right)$.

EXAMPLE 2.42. For $(f, s, \mathbf{x})$ as in Figure 8 and $I=\{1,3\}$, then the resulting tree is $\operatorname{Tree}_{ \pm}\left(f, s,\left\{x_{1}, x_{3}\right\}\right)=\left(t_{I}, \varepsilon_{I}\right)=\bigoplus$.

Getting to permutations, we can combine Observations 2.11 and 2.40 to obtain the following.

ObSERVATION 2.43. Let $(t, \varepsilon)$ be a signed Schröder tree, and $(f, s)$ be its signed contour. Consider a subset $I$ of the set of leaves of $t$, and denote by $\mathbf{x}$ the corresponding subset of $\boldsymbol{\ell}_{t}$. We have

$$
\operatorname{Perm}(f, s, \mathbf{x})=\operatorname{pat}_{I}(\operatorname{perm}(t, \varepsilon)) .
$$

EXAMPLE 2.44. We consider the signed Scröder tree $(t, \varepsilon)$ from Example 2.7 and the set $I=\{2,4,7\}$. We have in this case $\operatorname{pat}_{I}(\operatorname{perm}(t, \varepsilon))=123$. On the other hand, the construction of $\operatorname{Perm}(f, s, \mathbf{x})$ is illustrated on Figure 9 and also yields the permutation 123 . 


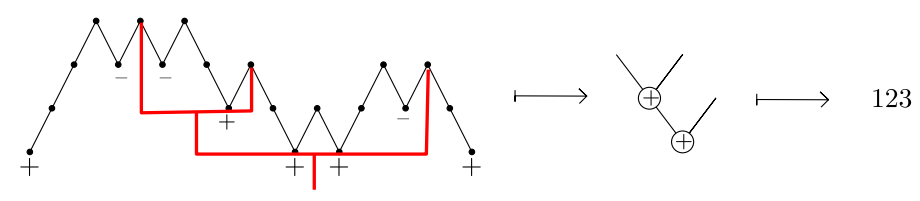

FIG. 9. Extracting a signed tree from the signed contour of a bigger tree and a subset of its leaves.

Another situation where we will extract a permutation from a signed excursion is the following.

OBSERVATION 2.45. Let $(f, s)$ be a realization of the signed Brownian excursion $(e, S)$ and $\mathbf{x}$ be a set of $k$ points taken uniformly and independently at random in $[0,1]$. Then Condition $(\mathcal{C})$ is satisfied with probability 1 for $(e, S, \mathbf{x})$, and the extracted tree is binary (see Lemmas A.1 and A.2 in the Appendix).

2.5. Measurability issues. In this section, we construct a suitable probability space and $\sigma$-algebra for the signed Brownian excursion. A possibility would be to take a Brownian excursion $e$ and a family of i.i.d. signs $\left(s_{x}\right)_{x \in[0,1]}$, independent from the excursion (and then consider only those indexed by minima of $e$ ). With this construction, the function $(f, s, \mathbf{x}) \mapsto \operatorname{Tree}_{ \pm}(f, s, \mathbf{x})$ would however not be measurable. We therefore give a slightly more subtle construction.

We fix an enumeration of the rational intervals in $[0,1]$, that is, a bijection

$$
\Psi: \mathbb{Z}_{>0} \rightarrow\left\{[p, q] \in \mathbb{Q}^{2}, 0 \leq p<q \leq 1\right\} .
$$

Denote $\Psi(i)=\left[p_{i}, q_{i}\right]$. Let $b$ be the position of a local minimum in an excursion $f$. We say that $b$ is associated with $\left[p_{i}, q_{i}\right]$ if $b=\operatorname{argmin}_{\left[p_{i}, q_{i}\right]} f$ and if $i$ is minimal with this property. Clearly, such an $i$ always exists.

To construct the signed Brownian excursion $(e, S)$, we now take a Brownian excursion $e$ and an i.i.d. sequence of balanced signs $\left(S_{i}\right)_{i \geq 1}$, independent from $e$. Consider a (strict) local minimum $b$ of $e$ and let $i$ be such that $b$ is associated with $\left[p_{i}, q_{i}\right]$. We then think as $S_{i}$ as being the sign of $b$.

A given interval $\left[p_{i}, q_{i}\right]$ can be associated with at most one $b$ (the position of the minimum of $e$ on $\left[p_{i}, q_{i}\right]$; by Lemma A.2, almost surely, this minimum is unique), which guarantees the independence of the signs of the different local minima. Note that some interval $\left[p_{i}, q_{i}\right]$ may be associated with no local minimum $b$ (when the position of the minimum of $e$ on $\left[p_{i}, q_{i}\right]$ is associated with $\left[p_{j}, q_{j}\right]$, for some $j<i$ ), which does not create any problem.

We claim that, on this probability space, the function $(f, s, \mathbf{x}) \mapsto \operatorname{Tree}_{ \pm}(f, s, \mathbf{x})$ is measurable. The unsigned version $(f, \mathbf{x}) \mapsto \operatorname{Tree}(f, \mathbf{x})$ is a classical object in the literature [30], Section 2.5 and it is easy to prove its measurability. The difficulty comes from the sign, and hence we focus on the case $|\mathbf{x}|=2$, the general one 
following easily. We have

$$
\begin{aligned}
& \left\{\left(f, s, x_{1}, x_{2}\right): \operatorname{Tree}_{ \pm}\left(f, s, x_{1}, x_{2}\right)=\bigoplus\right\} \\
& =\bigcup_{i \geq 1}\left\{\left(f, s, x_{1}, x_{2}\right): \operatorname{argmin}_{\left[x_{1} ; x_{2}\right]} f \text { is associated with }\left[p_{i}, q_{i}\right] \text { and } s_{i}=+\right\} .
\end{aligned}
$$

It is easy to check that this is a countable union of measurable sets, hence a measurable set itself. Therefore, our measurability claim is proved.

The function Perm is then also measurable since Perm $=$ perm $\circ$ Tree $_{ \pm}$. These are the only two functions of the signed Brownian excursion of interest for this paper.

\section{The variables $\Lambda_{\pi}$ : Definition, properties and proof schema of the main result.}

3.1. Description of the limit variables $\Lambda_{\pi}$. Recall from the Introduction that $\widetilde{\mathrm{occ}}\left(\pi, \sigma_{n}\right)$ denotes the proportion of occurrences of $\pi$ in a uniform random separable permutation of size $n$. Our main result [see Theorem 1.2(ii) and (iii), page 2138] is the (joint) convergence in distribution of $\widetilde{\text { occ }}\left(\pi, \sigma_{n}\right)$ towards a random variable $\Lambda_{\pi}$ that we now define.

We denote by $\mathbf{1}[A]$ the characteristic function of an event $A$.

DEFINITION 3.1. Let $\pi$ be a pattern and $(e, S)$ be the signed Brownian excursion. Let also $k=|\pi|$. Let $X_{1}, X_{2}, \ldots, X_{k}$ be $k$ uniform and independent points in $[0,1]$, independent from $(e, S)$ and set $\mathbf{X}=\left\{X_{1}, X_{2}, \ldots, X_{k}\right\}$. The random variable $\Lambda_{\pi}$ is defined by

$$
\Lambda_{\pi}=\mathbb{P}(\operatorname{Perm}(e, S, \mathbf{X})=\pi \mid e, S) .
$$

Note that this may be rephrased as $\Lambda_{\pi}=\mathbb{E}(\mathbf{1}[\operatorname{Perm}(e, S, \mathbf{X})=\pi] \mid e, S)$.

More precisely, we define the infinite-dimensional random vector $\left(\Lambda_{\pi}\right)_{\pi}$, indexed by all patterns $\pi$ of all sizes as follows: for each $\pi$, let $\mathbf{X}^{(\pi)}$ be a set of $|\pi|$ independent uniform random variables in $[0,1]$, taken independently for different patterns $\pi$, then we set

$$
\left(\Lambda_{\pi}\right)_{\pi}=\mathbb{E}\left(\left(\mathbf{1}\left[\operatorname{Perm}\left(e, S, \mathbf{X}^{(\pi)}\right)=\pi\right]\right)_{\pi} \mid e, S\right) .
$$

In this definition, and throughout the paper, the event "Perm $(e, S, \mathbf{X})=\pi$ " should be understood as "Perm $(e, S, \mathbf{X})$ is defined and is equal to $\pi$ ".

Since the finite-dimensional marginals of $\left(\Lambda_{\pi}\right)_{\pi}$ appear in our main theorem, rather than the whole vector, let us write down explicitly the definition of these finite-dimensional laws: for any patterns $\pi_{1}, \ldots, \pi_{r}$ we have

$$
\begin{aligned}
\left(\Lambda_{\pi_{1}}, \ldots, \Lambda_{\pi_{r}}\right)= & \mathbb{E}\left(\left(\mathbf{1}\left[\operatorname{Perm}\left(e, S, \mathbf{X}^{\left(\pi_{1}\right)}\right)=\pi_{1}\right], \ldots,\right.\right. \\
& \left.\left.\mathbf{1}\left[\operatorname{Perm}\left(e, S, \mathbf{X}^{\left(\pi_{r}\right)}\right)=\pi_{r}\right]\right) \mid e, S\right) .
\end{aligned}
$$


Note that in the definition of $\Lambda_{\pi}$, we condition on the random variable $(e, S)$, so that $\Lambda_{\pi}$ is a random variable itself. Moreover, in (2) and (3), all coordinates are defined by conditioning on the same realization $(e, S)$. Thus, the variables $\Lambda_{\pi}$ corresponding to different patterns $\pi$ are not independent.

The reader less familiar with probability theory might be more comfortable with the following equivalent description of $\Lambda_{\pi}$. For simplicity, we only discuss below the definition of the one-dimensional random variable $\Lambda_{\pi}$ (for a fixed pattern $\pi$ ), and not of the full infinite-dimensional vector $\left(\Lambda_{\pi}\right)_{\pi}$.

Let us define a function $\Psi_{\pi}$ on signed excursions as follows:

$$
\Psi_{\pi}(f, s)=\mathbb{P}(\operatorname{Perm}(f, s, \mathbf{X})=\pi),
$$

where $\mathbf{X}$ is a set of $|\pi|$ uniform and independent points in $[0,1]$. Then we set $\Lambda_{\pi}:=\Psi_{\pi}(e, S)$ to be the image of the signed Brownian excursion by $\Psi_{\pi}$.

In other words, in (1), the probability is taken with respect to $\mathbf{X}$, while we consider a realization of $(e, S)$. In such situations, we will sometimes use a superscript on $\mathbb{P}$ to record the source of randomness: namely, we write (1) as

$$
\Lambda_{\pi}=\mathbb{P}^{\mathbf{X}}(\operatorname{Perm}(e, S, \mathbf{X})=\pi) \text {. }
$$

Similarly, we use superscripts on expectation symbols $\mathbb{E}$ to indicate the source of randomness.

OBSERVATION 3.2. With this notation, we have the obvious compatibility relation

$$
\mathbb{E}^{e, S}\left[\mathbb{E}^{\mathbf{X}}(g(e, S, \mathbf{X}))\right]=\mathbb{E}^{e, S, \mathbf{X}}[g(e, S, \mathbf{X})],
$$

for any function $g$ such that these quantities are defined. If $g$ is the indicator function of an event $A$, this can be rewritten as

$$
\mathbb{E}^{e, S}\left[\mathbb{P}^{\mathbf{X}}(A)\right]=\mathbb{P}^{e, S, \mathbf{X}}(A) .
$$

To finish this section, we discuss trivial cases of our main theorem, when $\pi$ is not separable and when $\pi$ is the permutation of size 1 .

REMARK 3.3. Observe that if $\pi$ is not a separable permutation, from Proposition 2.12 it cannot be obtained as $\operatorname{Perm}(e, S, \mathbf{X})$, and thus $\Lambda_{\pi}$ is identically equal to 0 in this case. Clearly, $\widetilde{\text { occ }}\left(\pi, \sigma_{n}\right)$ is also identically equal to 0 in this case since a separable permutation cannot have a nonseparable pattern (permutation classes are by definition downward-closed for the pattern relation).

REMARK 3.4. If $\pi=1$ is the only permutation of size 1 , then regardless of $\mathbf{X}=\left(X_{1}\right)$ we have that $\operatorname{Perm}(e, S, \mathbf{X})$ is the permutation of size 1 . Consequently, $\Lambda_{\pi}$ is identically equal to 1 in this case. Similarly, $\widetilde{\text { occ }}\left(\pi, \sigma_{n}\right)$ is also identically equal to 1 , since every element of a permutation is an occurrence of the pattern $\pi=1$.

Thus, our main theorem is vacuous in the special cases $\pi$ not separable or $\pi=1$. 
3.2. The leaf distribution function of a tree. Before going further, we need a detour through distribution functions, to encode the positions of the leaves in the renormalized contour of a tree $t$.

A distribution function $F$ is a right-continuous nondecreasing function from $\mathbb{R}$ to $[0,1]$ with $\lim _{x \rightarrow-\infty} F(x)=0$ and $\lim _{x \rightarrow+\infty} F(x)=1$. A real-valued random variable $X$ has distribution function $F$ if, for all $x$, one has $F(x)=P(X \leq x)$. In the sequel, we only consider distribution functions $F$ such that $F(0)=0$ and $F(1)=1$ (equivalently, the associated random variables have values in $[0,1]$ ); and we identify $F$ with its restriction on the domain $[0,1]$.

For such a distribution function $F$, the pseudo-inverse $F^{*}$ of $F$ is defined as follows: for $u$ in $[0,1]$, we set $F^{*}(u)=\inf \{x \in[0,1]: F(x) \geq u\}$. One can check that for all $u \in[0,1]$ and $\varepsilon>0$,

$$
F\left(F^{*}(u)\right) \geq u \geq F\left(F^{*}(u)-\varepsilon\right) .
$$

Moreover, if $U$ is a uniform random variable on $[0,1]$, then $F^{*}(U)$ has distribution function $F$.

The following distribution functions will be of particular interest in this work:

- For the uniform distribution on $[0,1]$, we have $F_{U}(x)=\min (x, 1) \mathbf{1}[x \geq 0]$.

- With any tree $t$ with set of leaves $\ell_{t}=\left\{\ell_{1}, \ldots, \ell_{|t|}\right\}(|t|>1)$, we associate its leaf distribution function $F_{t}$ defined by

$$
F_{t}(x)=\frac{1}{|t|} \sum_{i=1}^{|t|} \mathbf{1}\left[\ell_{i} \leq x\right] .
$$

By definition, taking a random variable with distribution $F_{t}$ corresponds to choosing a leaf of $t$ uniformly at random (more precisely the $x$-coordinate $\ell_{i}$ of the corresponding leaf in the normalized contour of $t$ ). Figure 10 shows the leaf distribution function associated with the (unsigned) tree of Figure 7, page 2148.

The following statement was essentially proved by Pitman and Rizzolo in [37].

PROPOSITION 3.5. Let $T_{n}$ be a uniform random Schröder tree with $n$ leaves. Defining the random distribution function $F_{T_{n}}$ as above, we have the following convergence in probability:

$$
\left\|F_{T_{n}}-F_{U}\right\|_{\infty} \rightarrow 0
$$

Informally, choosing uniformly at random a leaf in $T_{n}$ (or rather the corresponding $x$-coordinate in the normalized contour $\widetilde{C_{T_{n}}}$ ) amounts in the limit to choosing a uniform point in $[0,1]$.

ProOF OF Proposition 3.5. Recall from Proposition 2.15, that $T_{n}$ can be seen as a Galton-Watson tree with a specific offspring distribution, conditioned to have $n$ leaves. 


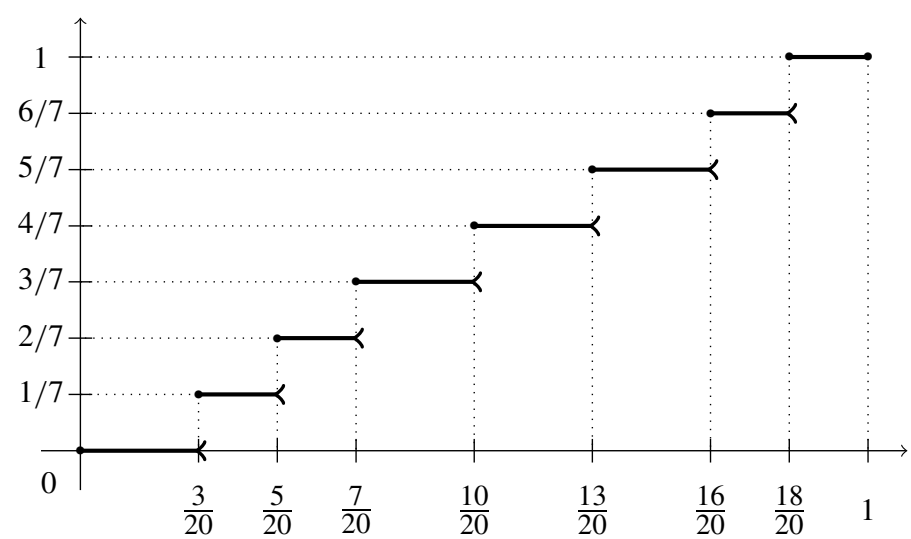

FIG. 10. The leaf distribution function $F_{t}$ for the tree $t$ shown in Figure 7. Note that $F_{t}$ is piecewise constant and all gaps are of height $\frac{1}{|t|}$ (here, $\frac{1}{7}$ ), but pieces may have different widths (here, $\frac{2}{20}$ or $\frac{3}{20}$ ). Informally, Proposition 3.5 states that these widths are asymptotically close to each other.

In the proof of [37], Theorem 8, Pitman and Rizzolo established for such models the convergence of the empirical distribution of the location of leaves (that they denote $v^{n}$ ) to the Lebesgue measure on $[0,1]$. This convergence holds in probability for the weak topology.

Weak convergence is equivalent to the convergence of distribution functions at continuity points of the limit, so that their statement correspond to the convergence of $F_{T_{n}}$ to $F_{U}$ in probability for the pointwise convergence topology. Moreover, since distribution functions are nondecreasing and since the limit $F_{U}$ is continuous, it is well known and easy to see that pointwise convergence implies uniform convergence, so that the proposition is proved.

We finish by a simple lemma, that is used in Sections 5 and 6.

LEMMA 3.6. If $F$ is a distribution function, then $\left\|F^{*}-F_{U}^{*}\right\|_{\infty} \leq\left\|F-F_{U}\right\|_{\infty}$.

Note that on $[0,1], F_{U}^{*}=F_{U}=\mathrm{id}_{[0,1]}$.

Proof of Lemma 3.6. Choose $u \in[0,1]$. If $u \geq F^{*}(u)$ then

$$
\begin{aligned}
\left|F^{*}(u)-F_{U}(u)\right| & =u-F^{*}(u) \leq F\left(F^{*}(u)\right)-F^{*}(u) \\
& \leq \sup _{t \in[0,1]}|F(t)-t|=\left\|F-F_{U}\right\|_{\infty}
\end{aligned}
$$

If on the contrary $u<F^{*}(u)$, then write for a small $\varepsilon>0$

$$
\left|F^{*}(u)-F_{U}(u)\right|=F^{*}(u)-u \leq F^{*}(u)-\varepsilon-F\left(F^{*}(u)-\varepsilon\right)+\varepsilon
$$




$$
\begin{aligned}
& \leq \sup _{t \in[-\varepsilon, 1-\varepsilon]}|t-F(t)|+\varepsilon \\
& \leq \varepsilon+\sup _{t \in[0,1-\varepsilon]}|t-F(t)|+\varepsilon \leq\left\|F-F_{U}\right\|_{\infty}+2 \varepsilon,
\end{aligned}
$$

and let $\varepsilon \rightarrow 0$.

3.3. Reformulation of the main theorem. To prove the convergence of the quantity $\widetilde{\mathrm{Occ}}\left(\pi, \sigma_{n}\right)$ towards $\Lambda_{\pi}$, it is useful to describe these two random variables in a similar manner. More precisely, we define a function $\operatorname{Pr}^{P e r m}$ in such a way that both $\widetilde{\text { occ }}\left(\pi, \sigma_{n}\right)$ and $\Lambda_{\pi}$ have a natural expression using $\operatorname{Pr}{ }^{P e r m}$. Along the way, we also define a tree analogue $\operatorname{Pr}^{\text {Tree }}$ of $\operatorname{Pr}^{\text {Perm }}$, which we shall use in the proof of Theorem 1.2(iii).

Definition 3.7 ( $\left.\operatorname{Pr}^{\text {Tree }}\right)$. Let $t_{0}$ be a tree with $k$ leaves, $f$ be an excursion and $F$ be a distribution function with $F(0)=0$ and $F(1)=1$. Let $X_{1}, \ldots, X_{k}$ be independent random variables with distribution function $F$ and $\mathbf{X}$ be the set $\left\{X_{1}, \ldots, X_{k}\right\}$. Then $\operatorname{Pr}^{\text {Tree }}\left(t_{0} ; f, F\right)=\mathbb{P}^{\mathbf{X}}\left(\operatorname{Tree}(f, \mathbf{X})=t_{0}\right)$.

DEFINITION $3.8\left(\mathrm{Pr}^{\mathrm{Perm}}\right)$. Let $\pi$ be a permutation of size $k,(f, s)$ be a signed excursion and $F$ be a distribution function with $F(0)=0$ and $F(1)=1$. Let $X_{1}, \ldots, X_{k}$ be independent random variables with distribution function $F$ and $\mathbf{X}$ be the set $\left\{X_{1}, \ldots, X_{k}\right\}$. Then $\operatorname{Pr}^{\operatorname{Perm}}(\pi ; f, s, F)=\mathbb{P}^{\mathbf{X}}(\operatorname{Perm}(f, s, \mathbf{X})=\pi)$.

Note that in the above two definitions, if the event $\operatorname{Tree}(f, \mathbf{X})=t_{0}$ [resp., $\operatorname{Perm}(f, s, \mathbf{X})=\pi]$ is realized, then there is no repetition in $X_{1}, \ldots, X_{k}$.

Observation 3.9. By definition, it follows that $\Lambda_{\pi}=\operatorname{Pr}^{\operatorname{Perm}}\left(\pi ; e, S, F_{U}\right)$.

LEMMA 3.10. Let $(t, \varepsilon)$ be a signed tree and $\sigma=\operatorname{perm}(t, \varepsilon)$ be the corresponding permutation. Let $\left(\widetilde{C}_{t}, s\right)$ be the signed contour of $(t, \varepsilon)$ and $F_{t}$ be the distribution function associated with $t$. It holds that

$$
\operatorname{Pr}^{\operatorname{Perm}}\left(\pi ; \widetilde{C}_{t}, s, F_{t}\right)=\frac{(|t|)_{k}}{|t|^{k}} \widetilde{\mathrm{occ}}(\pi, \sigma),
$$

where $(x)_{k}$ denotes the falling factorial $x(x-1) \cdots(x-k+1)$.

ProOF. Let $X_{1}, \ldots, X_{k}$ be independent random variables with distribution function $F_{t}$ [in this case, $\operatorname{Perm}\left(\widetilde{C_{t}}, s, \mathbf{X}\right)$ is always defined-see Observation 2.39]. In other terms, we take $k$ leaves of $t$ independently uniformly at random. The probability that they are different is clearly $(|t|)_{k} /|t|^{k}$. Conditioned to that event, $\mathbf{X}=\left\{X_{1}, \ldots, X_{k}\right\}$ is a uniform subset of $k$ leaves of $t$. From Observation 2.43, we have

$$
\operatorname{Perm}\left(\widetilde{C_{t}}, s, \mathbf{X}\right)=\operatorname{pat}_{I}(\sigma)
$$


where $I$ is a uniform random $k$-element subset of $[|t|]$. The probability that the right-hand side is equal to $\pi$ is by definition $\widetilde{\mathrm{occ}}(\pi, \sigma)$. Thus,

$$
\mathbb{P}\left(\operatorname{Perm}\left(\widetilde{C_{t}}, s, \mathbf{X}\right)=\pi \mid \mathbf{X} \text { is repetition-free }\right)=\widetilde{\operatorname{occ}}(\pi, \sigma),
$$

which completes the proof.

COROLlARY 3.11. Recall that $T_{n}$ denotes a uniform random Schröder tree with $n$ leaves, and $\mathcal{E}_{n}$ the sign function on the internal vertices of $T_{n}$, such that the signs alternate and the root of $T_{n}$ has a balanced sign. Denote by $\left(\widetilde{C_{T_{n}}}, S_{n}\right)$ the signed contour of $\left(T_{n}, \mathcal{E}_{n}\right)$. Then

$$
\widetilde{\mathrm{Occ}}\left(\pi, \sigma_{n}\right)=\operatorname{Pr}^{\operatorname{Perm}}\left(\pi ; \widetilde{C_{T_{n}}}, S_{n}, F_{T_{n}}\right)\left(1+\mathcal{O}\left(\frac{1}{n}\right)\right)
$$

Using Observation 3.9 and Corollary 3.11, our main result, that is, the joint convergence in distribution of $\widetilde{\mathrm{occ}}\left(\pi, \sigma_{n}\right)$ to $\Lambda_{\pi}$ [see Theorem 1.2(iii)] can now be written as the joint convergence (for any finite family of patterns $\pi$ ):

$$
\operatorname{Pr}^{\operatorname{Perm}}\left(\pi ; \widetilde{C_{T_{n}}}, S_{n}, F_{T_{n}}\right) \stackrel{d}{\rightarrow} \operatorname{Pr}^{\operatorname{Perm}}\left(\pi ; e, S, F_{U}\right),
$$

3.4. Outline of the proof. The proof of (5) consists in several steps, as follows. Recall that the normalized contour $\widetilde{C_{T_{n}}}$ and the leaf distribution function $F_{T_{n}}$ of $T_{n}$ converge to the Brownian excursion $e$ and the uniform distribution function $F_{U}$, respectively.

A natural way to proceed would be to prove the convergence of $\left(\widetilde{C_{T_{n}}}, S_{n}\right)$ to the signed Brownian excursion, and the continuity of $\operatorname{Pr}^{P e r m}(\pi ; \cdot)$. As discussed in Remark 2.29, a major difficulty when trying to prove that $\left(\widetilde{C_{T_{n}}}, S_{n}\right)$ converges to $(e, S)$ is that the signs on the local minima of $\widetilde{C_{T_{n}}}$ are far from independent. Instead of attacking this difficulty head-on, we have developed a proof along a different line. Recall that our goal is to prove the convergence in distribution of $\widetilde{\text { occ }}\left(\pi, \sigma_{n}\right)$ to $\Lambda_{\pi}$.

The main part of our proof is to show the convergence of $\widetilde{\mathrm{occ}}\left(\pi, \sigma_{n}\right)$ to $\Lambda_{\pi}$ in expectation. In Section 4, we prove that this is enough to have convergence in distribution. Indeed we shall see that all moments of $\Lambda_{\pi}$ are determined by expectations of $\Lambda_{\rho}$, for larger permutations $\rho$. Moreover, we shall see that a similar statement holds in the limit for $\widetilde{\text { occ }}\left(\pi, \sigma_{n}\right)$. Therefore, convergence of expectations implies convergence of all (joint) moments. And since the random variables are bounded by 1 , convergence of (joint) moments implies (multi-dimensional) convergence in distribution.

The goal of Section 5 is to prove the convergence in distribution of the unsigned trees extracted from the normalized contour of a uniform Schröder tree to the unsigned trees extracted from the Brownian excursion. This is achieved by proving 
the continuity of $\operatorname{Pr}^{\operatorname{Tree}}\left(t_{0} ; \cdot\right)$, and using the convergence of $\widetilde{C_{T_{n}}}$ to $e$ and the convergence of $F_{T_{n}}$ to $F_{U}$. Since this step does not involve signs, there is no major difficulty here.

In Section 6, we return to signed objects, proving that the signs on extracted trees are balanced and independent. More precisely, we examine the signs of the internal vertices of a signed tree $\left(t_{0}, \varepsilon\right)$ extracted from $\left(\widetilde{C_{T_{n}}}, S_{n}\right)$, and we prove that, when $n$ goes to infinity, these signs are balanced and independent. Note that a similar statement for $(e, S)$ is obvious, because the signs are chosen balanced and independent in $(e, S)$. The proof for $\left(\widetilde{C_{T_{n}}}, S_{n}\right)$ involves the fact that the relative distances between the points of $\widetilde{C_{T_{n}}}$ corresponding to the vertices of $t_{0}$ tend to infinity, and a subtree exchangeability argument.

Finally, we put all these results together in Section 7, to conclude the proof of Theorem 1.2(iii). More precisely, using Sections 5 and 6 we show the convergence of $\widetilde{\mathrm{occ}}\left(\pi, \sigma_{n}\right)$ to $\Lambda_{\pi}$ in expectation, which implies Theorem 1.2(iii) using Section 4.

4. Expectations determine joint moments. Recall that, for each $n, \sigma_{n}$ denotes a uniform random separable permutation of size $n$, and that we want to prove the convergence in distribution of the random variables $\widetilde{\text { occ }}\left(\pi, \sigma_{n}\right)$ to $\Lambda_{\pi}$. Since the random variables $\widetilde{\mathrm{occ}}\left(\pi, \sigma_{n}\right)$ and the candidate limit $\Lambda_{\pi}$ are bounded by 1 , (multi-dimensional) convergence in distribution is equivalent to convergence of (joint) moments. (This can be seen, e.g., as a consequence of the Stone-Weierstrass theorem, which ensures that polynomials are dense in the set of continuous functions from $[0,1]^{r}$ to $\mathbb{R}$.) Concretely, Theorem 1.2(iii) (page 2138) is equivalent to the following statement.

THEOREM 4.1. For any list of patterns $\pi_{1}, \ldots, \pi_{r}$ (possibly with repetitions),

$$
\mathbb{E}\left[\prod_{i=1}^{r} \widetilde{\operatorname{occ}}\left(\pi_{i}, \sigma_{n}\right)\right] \longrightarrow \mathbb{E}\left[\prod_{i=1}^{r} \Lambda_{\pi_{i}}\right]
$$

Instead of proving a convergence in distribution of random variables, we can therefore limit ourselves to proving a convergence of real numbers, which is a lot more tractable. To make our task even simpler, we show in Corollary 4.6 below that Theorem 4.1 follows if we prove, for all $\pi$, the convergence of $\mathbb{E}\left[\widetilde{\mathrm{occ}}\left(\pi, \sigma_{n}\right)\right]$ to $\mathbb{E}\left[\Lambda_{\pi}\right]$. The key point is that $\prod_{i=1}^{r} \widetilde{\mathrm{occ}}\left(\pi_{i}, \sigma\right)$ can be expressed combinatorially as a linear combination of $\widetilde{\mathrm{occ}}(\rho, \sigma)$ for larger patterns $\rho$, and that the same relation holds between $\prod_{i=1}^{r} \Lambda_{\pi_{i}}$ and the $\Lambda_{\rho}$ 's.

DEFINITION 4.2. Let $\rho$ be a permutation of size $K$ and $\pi_{1}, \ldots, \pi_{r}$ be permutations such that $\sum_{i=1}^{r}\left|\pi_{i}\right|=K$. An ordered set-partition $\left(I_{i}\right)_{1 \leq i \leq r}$ of $\{1, \ldots, K\}$ is compatible with $\rho, \pi_{1}, \ldots, \pi_{r}$ if for all $i$, pat $I_{i}(\rho)=\pi_{i}$. 
The number of ordered set-partitions compatible with $\rho, \pi_{1}, \ldots, \pi_{r}$ is denoted by $d_{\pi_{1}, \ldots, \pi_{r}}^{\rho}$. We also consider the proportion

$$
c_{\pi_{1}, \ldots, \pi_{r}}^{\rho}=\frac{d_{\pi_{1}, \ldots, \pi_{r}}^{\rho}}{\left(\begin{array}{c}
K \\
\left|\pi_{1}\right|, \ldots,\left|\pi_{r}\right|
\end{array}\right)}
$$

of ordered set-partitions of $\{1, \ldots, K\}$ which are compatible with $\rho, \pi_{1}, \ldots, \pi_{r}$.

EXAMPLE 4.3. Let $\rho=1342, \pi_{1}=21$ and $\pi_{2}=12$. There are 6 ordered setpartitions of $\{1, \ldots, 4\}$, which are $(\{1,2\},\{3,4\}),(\{1,3\},\{2,4\}),(\{1,4\},\{2,3\})$, $(\{2,3\},\{1,4\}),(\{2,4\},\{1,3\}),(\{3,4\},\{1,2\})$. Let $\left(I_{1}, I_{2}\right)$ be one of the first four ordered pairs, then pat $I_{1}(\rho)=12 \neq \pi_{1}$ thus $\left(I_{1}, I_{2}\right)$ is not compatible with $\rho, \pi_{1}$, $\pi_{2}$. On the contrary, the last two pairs are compatible with $\rho, \pi_{1}, \pi_{2}$. Then $d_{21,12}^{1342}=$ 2 and $c_{21,12}^{1342}=1 / 3$.

Proposition 4.4. Fix a list of patterns $\pi_{1}, \ldots, \pi_{r}$, and denote by $K$ the sum of their sizes. Then

$$
\prod_{i=1}^{r} \Lambda_{\pi_{i}}=\sum_{\rho \in \mathfrak{S}_{K}} c_{\pi_{1}, \ldots, \pi_{r}}^{\rho} \Lambda_{\rho}
$$

Proof. We fix a realization $(e, S)$ of the Brownian excursion all along the proof. The main idea to prove the equality in Proposition 4.4 is to show that both parts represent the probability of the same event. First, we describe the event.

We fix a list of patterns $\pi_{1}, \ldots, \pi_{r}$ and we denote by $K$ the sum of their sizes. Let us take $K$ independent uniform random variables in $[0,1]$. We denote the $\left|\pi_{1}\right|$ first ones (in the order of sampling) by $X_{1}^{1}, \ldots, X_{\left|\pi_{1}\right|}^{1}$, the following ones $X_{1}^{2}, \ldots, X_{\left|\pi_{2}\right|}^{2}$, and so on until the $\left|\pi_{r}\right|$ last ones which are denoted $X_{1}^{r}, \ldots, X_{\left|\pi_{r}\right|}^{r}$. Let $\mathbf{X}^{(i)}=\left\{X_{j}^{i}|1 \leq j \leq| \pi_{i} \mid\right\}$ for all $i$ and $\mathbf{X}=\bigcup_{i=1}^{r} \mathbf{X}^{(i)}$. The event we are considering is then: for each $i, \operatorname{Perm}\left(e, S, \mathbf{X}^{(i)}\right)=\pi_{i}$. Since $(e, S)$ is a fixed realization of the Brownian excursion, all the probabilities below should be understood with respect to the random variables $\mathbf{X}$, as indicated by the notation $\mathbb{P}^{\mathbf{X}}$.

Let $\mathcal{P}^{\pi_{1}, \ldots, \pi_{r}}=\mathbb{P}^{\mathbf{X}}\left(\forall i, \operatorname{Perm}\left(e, S, \mathbf{X}^{(i)}\right)=\pi_{i}\right)$. Since the $X_{j}^{i}$ are independent, we have

$$
\mathcal{P}^{\pi_{1}, \ldots, \pi_{r}}=\prod_{i=1}^{r} \mathbb{P}^{\mathbf{X}}\left(\operatorname{Perm}\left(e, S, \mathbf{X}^{(i)}\right)=\pi_{i}\right)=\prod_{i=1}^{r} \Lambda_{\pi_{i}} .
$$

It remains to prove that $\mathcal{P}^{\pi_{1}, \ldots, \pi_{r}}=\sum_{\rho \in \mathfrak{S}_{K}} c_{\pi_{1}, \ldots, \pi_{r}}^{\rho} \Lambda_{\rho}$. We compute this probability by conditioning on the value of $\operatorname{Perm}(e, S, \mathbf{X})$. Since $(e, S, \mathbf{X})$ satisfies Condition $(\mathcal{C})$ (page 2152 ) with probability 1 (see Observation 2.45 , page 2154 ), the permutation $\operatorname{Perm}(e, S, \mathbf{X})$ is almost surely defined and has size $K$. Thus,

$$
\begin{aligned}
\mathcal{P}^{\pi_{1}, \ldots, \pi_{r}}= & \sum_{\rho \in \mathfrak{S}_{K}} \mathcal{P}_{\rho} \times \mathbb{P}^{\mathbf{X}}(\operatorname{Perm}(e, S, \mathbf{X})=\rho) \\
& \text { where } \mathcal{P}_{\rho}=\mathbb{P}^{\mathbf{X}}\left(\forall i, \operatorname{Perm}\left(e, S, \mathbf{X}^{(i)}\right)=\pi_{i} \mid \operatorname{Perm}(e, S, \mathbf{X})=\rho\right) .
\end{aligned}
$$


By definition, $\mathbb{P}^{\mathbf{X}}(\operatorname{Perm}(e, S, \mathbf{X})=\rho)=\Lambda_{\rho}$, so we just need to prove that $\mathcal{P}_{\rho}=$ $c_{\pi_{1}, \ldots, \pi_{r}}^{\rho}$ to complete the proof.

Consider a realization of the random variables $\mathbf{X}$. We say that $X_{j}^{i}$ has rank $k$ if it is the $k$ th smallest value among all variables of $\mathbf{X}$. Then we can associate with $\mathbf{X}$ an ordered set-partition of $\{1, \ldots, K\}$ that we denote abusively Part( $\mathbf{X})$ and that is defined as follows: the $i$ th subset of $\operatorname{Part}(\mathbf{X})$ is obtained from $\mathbf{X}^{(i)}$ by replacing each $X_{j}^{i}$ by its rank. For example,

$$
\operatorname{Part}(\{0.7,0.9,0.2\},\{0.5,0.8\},\{0.3\})=(\{1,4,6\},\{3,5\},\{2\})
$$

since $0.2<0.3<0.5<0.7<0.8<0.9$.

Let $\mathcal{I}$ be the set of all ordered set-partitions of $\{1, \ldots, K\}$ in $r$ subsets such that the $i$ th one has $\left|\pi_{i}\right|$ elements. Then, by conditioning on the value of $\operatorname{Part}(\mathbf{X})$, we have

$$
\begin{aligned}
\mathcal{P}_{\rho}= & \sum_{\left(I_{i}\right) \in \mathcal{I}} \mathbb{P}^{\mathbf{X}}\left(\operatorname{Part}(\mathbf{X})=\left(I_{i}\right)\right) \\
& \times \mathbb{P}^{\mathbf{X}}\left(\forall i, \operatorname{Perm}\left(e, S, \mathbf{X}^{(i)}\right)=\pi_{i} \mid \operatorname{Perm}(e, S, \mathbf{X})=\rho \text { and } \operatorname{Part}(\mathbf{X})=\left(I_{i}\right)\right) .
\end{aligned}
$$

Recall that, by construction, Perm $=$ perm $\circ$ Tree \pm . From Observations 2.41 (page 2153) and 2.11 (page 2146),

$$
\begin{aligned}
\mathbb{P}^{\mathbf{X}}\left(\forall i, \operatorname{Perm}\left(e, S, \mathbf{X}^{(i)}\right)=\pi_{i} \mid \operatorname{Perm}(e, S, \mathbf{X})=\rho \text { and } \operatorname{Part}(\mathbf{X})=\left(I_{i}\right)\right) \\
\quad= \begin{cases}1 & \text { if }\left(I_{i}\right) \text { is compatible with } \rho, \pi_{1}, \ldots, \pi_{r} ; \\
0 & \text { otherwise. }\end{cases}
\end{aligned}
$$

In addition, we claim that $\operatorname{Part}(\mathbf{X})$ is uniformly distributed in $\mathcal{I}$, that is, for each $\left(I_{i}\right)$ in $\mathcal{I}$, we have $\mathbb{P}^{\mathbf{X}}\left(\operatorname{Part}(\mathbf{X})=\left(I_{i}\right)\right)=1 /\left(_{\left|\pi_{1}\right|, \ldots,\left|\pi_{r}\right|}\right)$. Indeed each possible relative order of the $\left(X_{j}^{i}\right)$ occurs with the same probability. Moreover, the block sizes of ordered set-partitions in $\mathcal{I}$ are prescribed: $\operatorname{card}\left(I_{i}\right)=\left|\pi_{i}\right|$. Therefore, each ordered set-partition in $\mathcal{I}$ corresponds to the same number of relative orders of the variables $\left(X_{j}^{i}\right)$; and this proves our claim.

Finally, we obtain that $\mathcal{P}_{\rho}=d_{\pi_{1}, \ldots, \pi_{r}}^{\rho} /\left(\begin{array}{c}K \\ \left|\pi_{1}\right|, \ldots,\left|\pi_{r}\right|\end{array}\right)=c_{\pi_{1}, \ldots, \pi_{r}}^{\rho}$. Going back to (6), we get $\mathcal{P}^{\pi_{1}, \ldots, \pi_{r}}=\sum_{\rho \in \mathfrak{S}_{K}} c_{\pi_{1}, \ldots, \pi_{r}}^{\rho} \Lambda_{\rho}$, which concludes the proof.

PROPOSITION 4.5. Fix a list of patterns $\pi_{1}, \ldots, \pi_{r}$, and denote by $K$ the sum of their sizes. Then for any permutation $\sigma$ of size $n$,

$$
\prod_{i=1}^{r} \widetilde{\mathrm{occ}}\left(\pi_{i}, \sigma\right)=\sum_{\rho \in \mathfrak{S}_{K}} c_{\pi_{1}, \ldots, \pi_{r}}^{\rho} \widetilde{\mathrm{occ}}(\rho, \sigma)+\mathcal{O}\left(\frac{1}{n}\right) .
$$

The constant hidden in the $\mathcal{O}$ symbol does depend on $\pi_{1}, \ldots, \pi_{r}$, but is uniform on $\sigma$. 
PROOF. Let $k_{i}$ be the size of $\pi_{i}$. We denote by $S_{i}$ the set of $k_{i}$-element subsets, $I$, of $[n]$ such that $\operatorname{pat}_{I}(\sigma)=\pi_{i}$. Then

$$
\prod_{i=1}^{r} \widetilde{\operatorname{occ}}\left(\pi_{i}, \sigma\right)=\prod_{i=1}^{r} \frac{\operatorname{card}\left(S_{i}\right)}{\left(\begin{array}{l}
n \\
k_{i}
\end{array}\right)}=\frac{\prod_{i=1}^{r} \operatorname{card}\left(S_{i}\right)}{\prod_{i=1}^{r}\left(\begin{array}{l}
n \\
k_{i}
\end{array}\right)} .
$$

We set $S_{\rho}=\left\{\left(s_{1}, \ldots, s_{r}\right) \in S_{1} \times S_{2} \times \cdots \times S_{r}\right.$ such that $\operatorname{pat}_{I}(\sigma)=\rho$ for $I=$ $\left.\bigcup_{i=1}^{r} s_{i}\right\}$. Then

$$
\begin{aligned}
\prod_{i=1}^{r} \operatorname{card}\left(S_{i}\right) & =\operatorname{card}\left(S_{1} \times S_{2} \times \cdots \times S_{r}\right) \\
& =\operatorname{card}\left(\bigcup_{\rho \in \mathfrak{S}_{K}} S_{\rho}\right)+\operatorname{card}\left(S_{1} \times S_{2} \times \cdots \times S_{r} \backslash \bigcup_{\rho \in \mathfrak{S}_{K}} S_{\rho}\right) .
\end{aligned}
$$

Note that the sets $S_{\rho}$ are disjoints: indeed $S_{\rho} \cap S_{\rho^{\prime}} \neq \varnothing \Rightarrow \rho=\operatorname{pat}_{I}(\sigma)=\rho^{\prime}$. Therefore, denoting $R=S_{1} \times S_{2} \times \cdots \times S_{r} \backslash \bigcup_{\rho \in \mathfrak{S}_{K}} S_{\rho}$, we have

$$
\prod_{i=1}^{r} \operatorname{card}\left(S_{i}\right)=\sum_{\rho \in \mathfrak{S}_{K}} \operatorname{card}\left(S_{\rho}\right)+\operatorname{card}(R) .
$$

We first study $R$. Let $\left(s_{1}, \ldots, s_{r}\right) \in S_{1} \times S_{2} \times \cdots \times S_{r}$, and define $I=$ $\bigcup_{i=1}^{r} s_{i}$. Note that $\operatorname{card}(I) \leq K$, and that the inequality is strict if and only if $\left(s_{1}, \ldots, s_{r}\right) \in R$. Consequently, it holds that

$$
R=\left\{\left(s_{1}, \ldots, s_{r}\right) \in S_{1} \times S_{2} \times \cdots \times S_{r} \text { such that } \operatorname{card}\left(\bigcup_{i=1}^{r} s_{i}\right)<K\right\} .
$$

It follows that $\operatorname{card}(R)=\mathcal{O}\left(n^{K-1}\right)$, where the constant hidden in the $\mathcal{O}$ symbol depends on the $k_{i}$ 's but not on $n$. Since $\prod_{i=1}^{r}\left(\begin{array}{l}n \\ k_{i}\end{array}\right) \sim \operatorname{cst} n^{K}$ for some constant cst that depends on the $k_{i}$ 's only, we obtain that $\frac{\operatorname{card}(R)}{\prod_{i=1}^{r}\left(\begin{array}{c}n \\ k_{i}\end{array}\right)}=\mathcal{O}\left(\frac{1}{n}\right)$, where the constant hidden in the $\mathcal{O}$ symbol depends on the $k_{i}$ 's only.

We now study $S_{\rho}$. Each element of $S_{\rho}$ corresponds to an occurrence of $\rho$ in $\sigma$. Conversely, a given occurrence of $\rho$ in $\sigma$ may lead to one, several or no element(s) of $S_{\rho}$ : this depends on the number of ways to partition the set of indices of $\sigma$ corresponding to the occurrence of $\rho$ into an ordered sequence $\left(s_{1}, \ldots, s_{r}\right)$ of $r$ sets such that each $s_{i}$ induces the pattern $\pi_{i}$. The number of ways to do this ordered partition does not depend on the occurrence of $\rho$ that is considered, and there are by definition $d_{\pi_{1}, \ldots, \pi_{r}}^{\rho}$ ways to do this partition. Thus,

$$
\begin{aligned}
\operatorname{card}\left(S_{\rho}\right) & =d_{\pi_{1}, \ldots, \pi_{r}}^{\rho} \times \text { number of occurrences of } \rho \text { in } \sigma \\
& =\left(\begin{array}{c}
K \\
k_{1}, \ldots, k_{r}
\end{array}\right) c_{\pi_{1}, \ldots, \pi_{r}}^{\rho} \times\left(\begin{array}{l}
n \\
K
\end{array}\right) \widetilde{\operatorname{occ}}(\rho, \sigma) .
\end{aligned}
$$


Putting things together,

$$
\begin{aligned}
\prod_{i=1}^{r} \widetilde{\operatorname{occ}}\left(\pi_{i}, \sigma\right) & =\sum_{\rho \in \mathfrak{S}_{K}} \frac{\operatorname{card}\left(S_{\rho}\right)}{\prod_{i=1}^{r}\left(\begin{array}{c}
n \\
k_{i}
\end{array}\right)}+\frac{\operatorname{card}(R)}{\prod_{i=1}^{r}\left(\begin{array}{l}
n \\
k_{i}
\end{array}\right)} \\
& =\sum_{\rho \in \mathfrak{S}_{K}} c_{\pi_{1}, \ldots, \pi_{r}}^{\rho} \widetilde{\widetilde{c c}}(\rho, \sigma) \frac{\left(\begin{array}{c}
K \\
k_{1}, \ldots, k_{r}
\end{array}\right)\left(\begin{array}{l}
n \\
n
\end{array}\right)}{\prod_{i=1}^{r}\left(\begin{array}{l}
n \\
k_{i}
\end{array}\right)}+\mathcal{O}\left(\frac{1}{n}\right) .
\end{aligned}
$$

By simple computations, we have

$$
\frac{\left(\begin{array}{c}
K \\
k_{1}, \ldots, k_{r}
\end{array}\right)\left(\begin{array}{l}
n \\
K
\end{array}\right)}{\prod_{i=1}^{r}\left(\begin{array}{l}
n \\
k_{i}
\end{array}\right)}=\frac{n !}{K !(n-K) !} \frac{K !}{k_{1} ! \cdots k_{r} !} \frac{k_{1} !\left(n-k_{1}\right) !}{n !} \ldots \frac{k_{r} !\left(n-k_{r}\right) !}{n !}=1+\mathcal{O}\left(\frac{1}{n}\right) \text {, }
$$

where again the constant hidden in the $\mathcal{O}$ symbol depends on the $k_{i}$ 's only. To conclude, we need to remark that the sum $\sum_{\rho \in \mathfrak{S}_{K}} c_{\pi_{1}, \ldots, \pi_{r}}^{\rho} \widetilde{\mathrm{Occ}}(\rho, \sigma)$ is bounded independently of $n$; indeed, each term $c_{\pi_{1}, \ldots, \pi_{r}}^{\rho} \widetilde{\mathrm{Occ}}(\rho, \sigma)$ is bounded by 1 , and there are $K$ ! terms. It then follows that

$$
\prod_{i=1}^{r} \widetilde{\mathrm{occ}}\left(\pi_{i}, \sigma\right)=\sum_{\rho \in \mathfrak{S}_{K}} c_{\pi_{1}, \ldots, \pi_{r}}^{\rho} \widetilde{\mathrm{Occ}}(\rho, \sigma)+\mathcal{O}\left(\frac{1}{n}\right),
$$

where the constant hidden in the $\mathcal{O}$ symbol does depend on $\pi_{1}, \ldots, \pi_{r}$, but is uniform on $\sigma$.

COROLLARY 4.6. Theorem 4.1 [and therefore Theorem 1.2(iii)] is equivalent to the following statement:

$$
\text { For any pattern } \pi, \quad \mathbb{E}\left[\widetilde{\operatorname{occ}}\left(\pi, \sigma_{n}\right)\right] \longrightarrow \mathbb{E}\left[\Lambda_{\pi}\right] .
$$

PROOF. We assume that equation (7) holds. Let $\pi_{1}, \ldots, \pi_{r}$ be a list of patterns, and denote by $K$ the sum of their sizes. Then, from Propositions 4.4 and 4.5, we have

$$
\begin{aligned}
\mathbb{E}\left[\prod_{i=1}^{r} \widetilde{\operatorname{occ}}\left(\pi_{i}, \boldsymbol{\sigma}_{n}\right)\right] & =\sum_{\rho \in \mathfrak{S}_{K}} c_{\pi_{1}, \ldots, \pi_{r}}^{\rho} \mathbb{E}\left[\widetilde{\mathrm{occ}}\left(\rho, \boldsymbol{\sigma}_{n}\right)\right]+o(1) \\
& =\sum_{\rho \in \mathfrak{S}_{K}} c_{\pi_{1}, \ldots, \pi_{r}}^{\rho} \mathbb{E}\left[\Lambda_{\rho}\right]+o(1)=\mathbb{E}\left[\prod_{i=1}^{r} \Lambda_{\pi_{i}}\right]+o(1)
\end{aligned}
$$

Note that to get the second line, we used that $\sum_{\rho \in \mathfrak{S}_{K}} c_{\pi_{1}, \ldots, \pi_{r}}^{\rho}$ does not depend on $n$. The above computations show that equation (7) implies Theorem 4.1.

The next three sections focus on proving equation (7); the proof will be completed in Theorem 7.3. 
5. Continuity of $\mathbf{P r}^{\text {Tree }}$. In this section, we prove the convergence in distribution of the trees extracted from the normalized contour of a uniform Schröder tree to the trees extracted from the Brownian excursion (in the unsigned case). This result is stated in Corollary 5.4. It follows easily from earlier results and a continuity property of $\operatorname{Pr}^{\text {Tree }}$ (defined in Definition 3.7, page 2159) that we establish in Lemma 5.2 below. Let us first set up some notation.

Fix a tree $t_{0}$ with $k$ leaves. We consider the map $(f, F) \mapsto \operatorname{Pr}^{\operatorname{Tree}}\left(t_{0} ; f, F\right)$. We use the uniform topology (i.e., the topology induced by the supremum norm) both on the set of excursions and on the set of distribution functions.

Throughout the section, if $f$ is an excursion and $x \leq y$ in $[0,1]$, we denote $m(f ; x, y)=\min _{[x, y]} f$. Let $X_{1} \leq X_{2} \cdots \leq X_{k}$ be the reordering of $k$ uniform i.i.d. random variables in $[0,1]$. We say that an excursion $f$ has the distinct minima property if $m\left(f, X_{1}, X_{2}\right), \ldots, m\left(f, X_{k-1}, X_{k}\right)$ are distinct with probability 1 (the probability here is taken with respect to $X_{1}, \ldots, X_{k}$ ).

LEMMA 5.1. Let $e$ be the Brownian excursion, then a.s. $e$ has the distinct minima property.

PROOF. This can be seen as a consequence of Lemma A.4, but since this lemma uses a deep result of [30], let us give a more elementary proof.

If $e$ is the Brownian excursion, and $X_{1}<\cdots<X_{k}$ is the reordering of $k$ i.i.d. uniform random variables in $[0,1]$, then from Lemma A.1 with probability 1 no $X_{i}$ is a one-sided minimum. When this is the case, for each $i$, $m\left(f, X_{i}, X_{i+1}\right)$ cannot be reached on the extremities of the interval $\left[X_{i}, X_{i+1}\right]$ and is therefore a local minimum. Consequently, using Lemma A.2, the values $m\left(f, X_{1}, X_{2}\right), \ldots, m\left(f, X_{k-1}, X_{k}\right)$ are distinct, almost surely.

LEMMA 5.2. Let $t_{0}$ be a fixed tree with $k$ leaves. If $f$ is an excursion with the distinct minima property, then $\operatorname{Pr}^{\operatorname{Tree}}\left(t_{0} ; \ldots\right)$ is continuous in $\left(f, F_{U}\right)$ with respect to the uniform topology.

Proof. We prove that $\operatorname{Pr}^{\operatorname{Tree}}\left(t_{0} ; g, G\right) \rightarrow \operatorname{Pr}^{\operatorname{Tree}}\left(t_{0} ; f, F_{U}\right)$ as soon as $g \rightarrow f$ and $G \rightarrow F_{U}$. Fix $\varepsilon, \delta>0$ and consider an excursion $g$ and a distribution function $G$ with $\|f-g\|_{\infty} \leq \varepsilon$ and $\left\|F_{U}-G\right\|_{\infty} \leq \delta$.

Recall from Lemma 3.6 (page 2158) that $\left\|F_{U}^{\star}-G^{\star}\right\|_{\infty} \leq\left\|F_{U}-G\right\|_{\infty} \leq \delta$ and that $F_{U}^{\star}$ is the identity on $[0,1]$. Let $X_{1}<\cdots<X_{k}$ be the reordering of $k$ independent uniform random variables, and let $\mathbf{x}=\left(X_{1}, \ldots, X_{k}\right)$. Furthermore, set $Y_{i}=G^{\star}\left(X_{i}\right)$. Note that $G^{\star}$ is nondecreasing, so that $Y_{1} \leq \cdots \leq Y_{k}$. We write $\mathbf{y}=\left(Y_{1}, \ldots, Y_{k}\right)$. By construction, we have that:

- $\mathbf{y}$ has the distribution of the reordering of $k$ i.i.d. random variables of distribution $G$;

- and, for each $i$, one has $\left|Y_{i}-X_{i}\right| \leq \delta$. 
It follows from $\|f-g\|_{\infty} \leq \varepsilon$ that $\left|m\left(g, Y_{i}, Y_{i+1}\right)-m\left(f, Y_{i}, Y_{i+1}\right)\right| \leq \varepsilon$ for $i \leq$ $k-1$. This implies that, for each $i \leq k-1$,

$$
\left|m\left(g, Y_{i}, Y_{i+1}\right)-m\left(f, X_{i}, X_{i+1}\right)\right| \leq \varepsilon+\omega(f, \delta),
$$

where $\omega$ is the modulus of continuity of $f$ defined by

$$
\omega(f, \delta)=\sup _{|r-s| \leq \delta}|f(r)-f(s)| .
$$

Recall that Tree $(f, \mathbf{x})$ is the tree extracted from the set of points $\mathbf{x}$ on the excursion $f$. We have

$$
\begin{aligned}
& \left|\operatorname{Pr}^{\text {Tree }}\left(t_{0} ; f, F_{U}\right)-\operatorname{Pr}^{\text {Tree }}\left(t_{0} ; g, G\right)\right| \\
& \quad=\left|\mathbb{P}^{\mathbf{x}}\left(\operatorname{Tree}(f, \mathbf{x})=t_{0}\right)-\mathbb{P}^{\mathbf{x}}\left(\operatorname{Tree}(g, \mathbf{y})=t_{0}\right)\right| \\
& \quad \leq \mathbb{P}^{\mathbf{x}}(\operatorname{Tree}(f, \mathbf{x}) \neq \operatorname{Tree}(g, \mathbf{y}))
\end{aligned}
$$

Above and in what follows, the randomness is only given by $\mathbf{x}$, the variables $\mathbf{y}$ are functions of the $\mathbf{x}$ 's, and hence random as well, while $f$ is nonrandom. We emphasize this by using the notation $\mathbb{P}^{\mathbf{x}}$.

By construction (see Definition 2.30, page 2151), the tree $\operatorname{Tree}(f, \mathbf{x})$ only depends on the relative order of $m\left(f, X_{1}, X_{2}\right), \ldots, m\left(f, X_{k-1}, X_{k}\right)$. We denote $\operatorname{gap}(f, \mathbf{x})$ the minimal difference between any two of these values. Since $f$ is assumed to have the distinct minima property, $\operatorname{gap}(f, \mathbf{x})$ is nonzero with probability 1 .

From (8), we see that the numbers $m\left(g, Y_{1}, Y_{2}\right), \ldots, m\left(g, Y_{k-1}, Y_{k}\right)$ are in the same relative order as $m\left(f, X_{1}, X_{2}\right), \ldots, m\left(f, X_{k-1}, X_{k}\right)$ as soon as we have $\varepsilon+$ $\omega(f, \delta)<\operatorname{gap}(f, \mathbf{x}) / 2$. If this is the case, then $\operatorname{Tree}(f, \mathbf{x})=\operatorname{Tree}(g, \mathbf{y})$. Therefore,

$$
\begin{aligned}
\mathbb{P}^{\mathbf{x}}(\operatorname{Tree}(f, \mathbf{x}) \neq \operatorname{Tree}(g, \mathbf{y})) & \leq \mathbb{P}^{\mathbf{x}}(\operatorname{gap}(f, \mathbf{x}) / 2 \leq \varepsilon+\omega(f, \delta)) \\
& \stackrel{\varepsilon, \delta \rightarrow 0}{\longrightarrow} \mathbb{P}^{\mathbf{x}}(\operatorname{gap}(f, \mathbf{x})=0)=0 .
\end{aligned}
$$

With (10), this completes the proof.

By definition, the (substitution) decomposition tree of $\sigma$ is the unique signed Schröder tree $(t, \varepsilon)$ in which the signs alternate such that $\operatorname{perm}(t, \varepsilon)=\sigma$. By Corollary 2.14 (page 2147), in the case where $\sigma_{n}$ is a uniform random separable permutation of size $n$, then its (unsigned) decomposition tree is a uniform Schröder tree with $n$ leaves, denoted $T_{n}$ all along the article.

PROPOSITION 5.3. Let $\sigma_{n}$ be a uniform random separable permutation of size $n$ and $\left(\widetilde{C_{T_{n}}}\right)$ be the normalized contour of its decomposition tree. We also define $F_{T_{n}}$ as usual [see (4), page 2157]. Let e be the Brownian excursion and define $\lambda=\sqrt{2+3 / \sqrt{2}}$. Then we have

$$
\left(\widetilde{C_{T_{n}}}, F_{T_{n}}\right) \stackrel{d}{\rightarrow}\left(\lambda \cdot e, F_{U}\right)
$$

in distribution, with respect to the uniform topology. 
PROOF. From Propositions 2.23 (page 2149) and 3.5 (page 2157), we know that $\widetilde{C_{T_{n}}} \stackrel{d}{\rightarrow} \lambda \cdot e$ and $F_{T_{n}} \stackrel{d}{\rightarrow} F_{U}$. It remains to prove the joint convergence. Note that the limit $F_{U}$ of $F_{T_{n}}$ is deterministic.

Thus, we want to use a theorem of Billingsley ([8], Theorem 3.9) that asserts that if $X_{n}^{\prime} \stackrel{d}{\rightarrow} X^{\prime}$ and $X_{n}^{\prime \prime} \stackrel{d}{\rightarrow} a^{\prime \prime}$ (where $X_{n}^{\prime}$ and $X^{\prime}$ are random variables with values in a metric space $S^{\prime}, X_{n}^{\prime \prime}$ are random variables with values in a metric space $S^{\prime \prime}$ and $a^{\prime \prime}$ is a deterministic element of $\left.S^{\prime \prime}\right)$, then $\left(X_{n}^{\prime}, X_{n}^{\prime \prime}\right) \stackrel{d}{\rightarrow}\left(x^{\prime}, a^{\prime \prime}\right)$, provided that $T=S^{\prime} \times S^{\prime \prime}$ is separable.

The hypothesis that $T$ is separable is in fact only needed to ensure that the Borel $\sigma$-algebra on $T$ is the product of the Borel $\sigma$-algebras on $S^{\prime}$ and $S^{\prime \prime}$. For this to be the case, it is sufficient that either $S^{\prime}$ or $S^{\prime \prime}$ is separable; see [9], Lemma 6.4.2.

In our case, the functions $\widetilde{C_{T_{n}}}$ and the limit $\lambda \cdot e$ are random elements in the space $S^{\prime}=\mathcal{C}[0,1]$ of continuous functions on $[0,1]$, which is known to be separable (see [8], Example 1.3). We can therefore use Billingsley's theorem and the proposition is proved.

COROLlARY 5.4. With the above notation, for any fixed $t_{0}$, we have the convergence in distribution:

$$
\operatorname{Pr}^{\operatorname{Tree}}\left(t_{0} ; \widetilde{C_{T_{n}}}, F_{T_{n}}\right) \stackrel{d}{\rightarrow} \operatorname{Pr}^{\text {Tree }}\left(t_{0} ; e, F_{U}\right)
$$

In particular, since these random variables are bounded, we have

$$
\mathbb{E}^{\sigma_{n}}\left[\operatorname{Pr}^{\text {Tree }}\left(t_{0} ; \widetilde{C_{T_{n}}}, F_{T_{n}}\right)\right] \rightarrow \mathbb{E}^{e}\left[\operatorname{Pr}^{\text {Tree }}\left(t_{0} ; e, F_{U}\right)\right]
$$

PROOF. This corollary is a simple application of the mapping theorem [8], Theorem 2.7 because of the following facts:

- $\left(\widetilde{C_{T_{n}}}, F_{T_{n}}\right) \stackrel{d}{\rightarrow}\left(\lambda \cdot e, F_{U}\right)$, which is proved in Proposition 5.3;

- $\operatorname{Pr}^{\operatorname{Tree}}\left(t_{0} ; \ldots\right)$ is invariant when the first argument is multiplied by a constant and is continuous at $\left(e, F_{U}\right)$ for almost every $e$, which follows from Lemmas 5.1 and 5.2.

6. Signs are asymptotically balanced and independent. We now return to the study of signed trees. The purpose of this section is to justify that asymptotically (when $n$ goes large) the $k-1$ signs in the tree Tree $\pm\left(\widetilde{C_{T_{n}}}, S_{n}, \ell\right)$ are independent and balanced, when $\ell$ is a $k$-element subset of the set of leaves of $T_{n}$ chosen uniformly at random.

PROPOSITION 6.1. Let $\sigma_{n}$ be a uniform random separable permutation of size $n$ and $\left(t_{0}, \varepsilon\right)$ be a signed binary tree of size $k$. As usual, let $\left(\widetilde{C_{T_{n}}}, S_{n}\right)$ be the signed contour of the decomposition tree of $\sigma_{n}$. We also consider a uniform random $k$-element subset $\ell$ of leaves of $T_{n}$. Then

$$
\mathbb{P}^{\sigma_{n}, \ell}\left(\operatorname{Tree}_{ \pm}\left(\widetilde{C_{T_{n}}}, S_{n}, \ell\right)=\left(t_{0}, \varepsilon\right)\right)=\frac{1}{2^{k-1}} \mathbb{P}^{\sigma_{n}, \ell}\left(\operatorname{Tree}\left(\widetilde{C_{T_{n}}}, \ell\right)=t_{0}\right)+o(1)
$$


The proof is given at the end of the present section. The core of this proof is Lemma 6.2 below, regarding heights of branching points of marked leaves in uniform random Schröder trees. We first introduce notation.

Recall that $T_{n}$ is a uniform Schröder tree of size $n$. We take a set of $k$ leaves of $T_{n}$ uniformly at random. As in Definition 2.21 (page 2149), denote their $x$ coordinates by $\ell_{1}<\cdots<\ell_{k}$, and $\ell=\left(\ell_{1}, \ldots, \ell_{k}\right)$ and let $H_{i}$ be the height of $\ell_{i}$ in $T_{n}$. For $i=1, \ldots, k-1$, denote by $M_{i}$ the height of the common ancestor of the leaves $\ell_{i}$ and $\ell_{i+1}$ (defining the height as the distance from the root).

LEMMA 6.2. When $n \rightarrow+\infty$, the parities of $M_{1}, \ldots, M_{k-1}$ are asymptotically balanced, independent from each other and from the subtree $t_{0}$ induced by the $k$ leaves.

More formally, we fix a binary tree $t_{0}$ with $k$ leaves and we condition on the event that the subtree of $T_{n}$ induced by $\ell$ is $t_{0}$. For all $\delta_{1}, \ldots, \delta_{k-1} \in\{0,1\}$,

$$
\mathbb{P}\left(M_{1} \equiv \delta_{1}, \ldots, M_{k-1} \equiv \delta_{k-1} \mid \operatorname{Tree}\left(\widetilde{C_{T_{n}}}, \ell\right)=t_{0}\right) \stackrel{n \rightarrow+\infty}{\longrightarrow} \frac{1}{2^{k-1}}
$$

where $a \equiv b$ means $a=b \bmod 2$.

PROOF. The proof essentially relies on a subtree exchangeability argument. In order to give intuition, we start by the case $k=2$.

In this case, there is a unique binary tree with two leaves, so the conditioning is not relevant. We want to prove that the common ancestor of two leaves $\ell_{1}$ and $\ell_{2}$, chosen uniformly at random in $T_{n}$ among all 2-element subsets of leaves, has an even height with probability asymptotically $1 / 2$.

Fix two integers $n, h \geq 1$. We consider the set $\mathcal{T}_{n}^{h}$ of Schröder trees $t$ with $n$ leaves, with two marked leaves $\ell_{1}$ and $\ell_{2}\left(\ell_{1}<\ell_{2}\right)$, such that $\ell_{1}$ has height $h$. Such a tree $t$ can be canonically described as follows:

- Take a chain $v_{0}, \ldots, v_{h}=\ell_{1}$ going from the root of $t$ to the first marked leaf.

- Then for each $x<h$, glue a tree $T_{x}^{l}$ and a tree $T_{x}^{r}$, respectively, on the left and on the right of $v_{x}$.

- One of the trees $T_{x}^{r}$, say $T_{x_{0}}^{r}$, contains the second marked leaf $\ell_{2}$. Then the vertex $v_{x_{0}}$ is the common ancestor of $\ell_{1}$ and $\ell_{2}$ and has height $M_{1}=x_{0}$.

For every permutation $\alpha$ of $\{0,1, \ldots, h-1\}$, replacing each pair $\left(T_{x}^{l}, T_{x}^{r}\right)$ by $\left(T_{\alpha(x)}^{l}, T_{\alpha(x)}^{r}\right)$ in the above decomposition provides a bijection from $\mathcal{T}_{n}^{h}$ to itself. This bijection maps a tree with $M_{1}=x_{0}$ onto a tree with $M_{1}=\alpha\left(x_{0}\right)$.

Applying this construction to our random tree $T_{n}$ with its two marked leaves, we get that for each $h, x$ with $0 \leq x \leq h-1$

$$
\mathbb{P}\left(M_{1}=x \mid H_{1}=h\right)=\frac{1}{h} .
$$



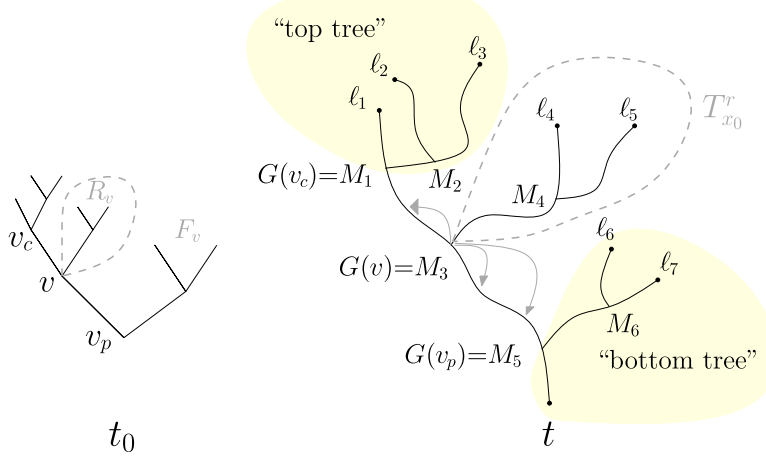

FIG. 11. The subtree exchangeability argument.

Therefore, we obtain

$$
\begin{aligned}
\mathbb{P}\left(M_{1} \text { is odd }\right) & =\sum_{h \geq 1} \mathbb{P}\left(M_{1} \text { is odd } \mid H_{1}=h\right) \mathbb{P}\left(H_{1}=h\right) \\
& =\sum_{h \geq 1} \frac{\lfloor h / 2\rfloor}{h} \mathbb{P}\left(H_{1}=h\right)=\frac{1}{2}-\mathcal{O}\left(\mathbb{E}\left(\frac{1}{H_{1}}\right)\right),
\end{aligned}
$$

which goes to $1 / 2$, as soon as $H_{1}$ goes to infinity in probability. This will be proved in Lemma 6.3 below.

We now prove Lemma 6.2 in the general case $k \geq 2$. Since the proof uses a lot of notation, the reader is invited to look regularly at Figure 11, which illustrates the main definitions.

We fix $k \geq 2$ and a binary tree $t_{0}$ with $k$ leaves. We denote by $\mathcal{T}_{n}\left(t_{0}\right)$ the set of Schröder trees of size $n$ with $k$ marked leaves $\ell_{1}<\cdots<\ell_{k}$, which induce the subtree $t_{0}$. For such a tree $t$, each vertex $v$ in $t_{0}$ corresponds to a vertex $G(v)$ in $t$ : $G(v)$ is either one of the marked leaves of $t$ (if $v$ is a leaf of $t_{0}$ ) or a common ancestor of two marked leaves of $t$ (if $v$ is an internal vertex of $t_{0}$ ). We denote by $h_{t}(v)$ the height of $G(v)$ in $t$.

We fix an internal vertex $v$ of $t_{0}$. Define $v_{c}$ as the left child of $v$ in $t_{0}$ and $v_{p}$ as the parent of $v$. For $t$ in $\mathcal{T}_{n}\left(t_{0}\right)$, the distance between $G\left(v_{c}\right)$ and $G\left(v_{p}\right)$ is $d_{t}(v):=h_{t}\left(v_{c}\right)-h_{t}\left(v_{p}\right)$. [If $v$ is the root, then $v_{p}$ is not defined and we shall think of $G\left(v_{p}\right)$ as a virtual new root of $t$ that is an ancestor of every vertex in $t$ and has height -1 ; in particular, we take $h_{t}\left(v_{p}\right)=-1$.]

The vertices of $t_{0}$ (both leaves and internal vertices, $v$ excluded) are partitioned in two sets: the right subtree $R_{v}$ of $v$ and its complement set $F_{v}$. For a sequence of heights $\mathbf{h}=\left(h_{u}\right)_{u \in F_{v}}$, we define

$$
\mathcal{T}_{n}^{v, \mathbf{h}}\left(t_{0}\right)=\left\{t \in \mathcal{T}_{n}\left(t_{0}\right), \text { for all } u \in F_{v}, h_{t}(u)=h_{u}\right\} .
$$

Note that, for every $t$ in this set, $d_{t}(v)=h_{v_{c}}-h_{v_{p}}$; we denote this common value by $d_{\mathbf{h}, v}$. (If $v$ is the root of $t_{0}$, we set $h_{v_{p}}=-1$.) 
Similar to the decomposition in the case $k=2$, each tree in $\mathcal{T}_{n}^{v, \mathbf{h}}\left(t_{0}\right)$ can be decomposed as follows:

- A chain $w_{0}, \ldots, w_{d_{\mathbf{h}, v}}$ going from $G\left(v_{p}\right)$ to $G\left(v_{c}\right)$.

- For each $0<x<d_{\mathbf{h}, v}$, a tree $T_{x}^{l}$ and a tree $T_{x}^{r}$, respectively, on the left and on the right of $w_{x}$.

- One of the trees $T_{x}^{r}$, say $T_{x_{0}}^{r}$, contains $G\left(R_{v}\right)$. Then the vertex $w_{x_{0}}$ is $G(v)$ and has height $h_{v_{p}}+x_{0}$.

- A "top tree" which consists of the offspring of $G\left(v_{c}\right)$.

- All others vertices of $t$ form a connected subtree called the "bottom tree". (If $v$ is the root of $t_{0}$, there is no bottom tree.)

For every permutation $\alpha$ of $\left\{1, \ldots, d_{\mathbf{h}, v}-1\right\}$, replacing each pair $\left(T_{x}^{l}, T_{x}^{r}\right)$ by $\left(T_{\alpha(x)}^{l}, T_{\alpha(x)}^{r}\right)$ in the above decomposition provides a bijection from $\mathcal{T}_{n}^{v, \mathbf{h}}\left(t_{0}\right)$ to itself. Clearly, this bijection maps a tree with $h_{t}(v)=h_{v_{p}}+x_{0}$ onto a tree with $h_{t}(v)=h_{v_{p}}+\alpha\left(x_{0}\right)$.

We now work with a uniform Schröder tree $T_{n}$ of size $n$ with $k$ marked leaves, and we condition on the fact that these leaves induce the subtree $t_{0}$. We use the notation $\mathbb{P}_{t_{0}}\left(T_{n} \in A\right):=\mathbb{P}\left(T_{n} \in A \mid T_{n} \in \mathcal{T}_{n}\left(t_{0}\right)\right)$. The above discussion implies that for every $h_{v_{p}}<x<h_{v_{c}}$ we have

$$
\mathbb{P}_{t_{0}}\left(h_{T_{n}}(v)=x \mid T_{n} \in \mathcal{T}_{n}^{v, \mathbf{h}}\left(t_{0}\right)\right)=\frac{1}{h_{v_{c}}-h_{v_{p}}-1}=\frac{1}{d_{\mathbf{h}, v}-1}=\frac{1}{d_{T_{n}}(v)-1} .
$$

Therefore,

$$
\mid \mathbb{P}_{t_{0}}\left(h_{T_{n}}(v) \text { is even } \mid T_{n} \in \mathcal{T}_{n}^{v, \mathbf{h}}\left(t_{0}\right)\right)-\frac{1}{2} \mid \leq \frac{1}{d_{T_{n}}(v)-1} .
$$

We introduce a subset $E_{n}^{v}$ of $\mathcal{T}_{n}\left(t_{0}\right)$ of well-behaved trees:

$$
E_{n}^{v}:=\left\{t \in \mathcal{T}_{n}\left(t_{0}\right), d_{t}(v) \geq n^{1 / 4}\right\} .
$$

We will prove in Lemma 6.3 that for all $v, \mathbb{P}_{t_{0}}\left(E_{n}^{v}\right) \rightarrow 1$.

Note that if $\mathbf{h}$ is such that $\mathcal{T}_{n}^{v, \mathbf{h}}\left(t_{0}\right) \subset E_{n}^{v}$, then

$$
\mid \mathbb{P}_{t_{0}}\left(h_{T_{n}}(v) \text { is even } \mid T_{n} \in \mathcal{T}_{n}^{v, \mathbf{h}}\left(t_{0}\right)\right)-\frac{1}{2} \mid \leq \frac{1}{n^{1 / 4}-1} .
$$

As a consequence, we have

$$
\mathbb{P}_{t_{0}}\left(h_{T_{n}}(v) \text { is even } \mid T_{n} \in \mathcal{T}_{n}^{v, \mathbf{h}}\left(t_{0}\right)\right)=\frac{1}{2}+o(1),
$$

where the error term $o(1)$ is bounded independently of $\mathbf{h}$, provided that we have $\mathcal{T}_{n}^{v, \mathbf{h}}\left(t_{0}\right) \subset E_{n}^{v}$.

Internal vertices of $t_{0}$ can be canonically indexed by $\{1, \ldots, k-1\}: v_{i}$ is the common ancestor of $\ell_{i}, \ell_{i+1}$. By definition, $M_{i}=h_{t}\left(v_{i}\right)$ and we denote $E_{n}^{i}:=E_{n}^{v_{i}}$. 
Fix $i \geq 1$. For $j<i$, the vertex $v_{j}$ lies in $F_{v_{i}}$, so that the fact that $T_{n}$ belongs to $\mathcal{T}_{n}^{v_{i}, \mathbf{h}}\left(t_{0}\right)$ determines the random variables $\left(M_{j}\right)_{j<i}$. Clearly, this also determines whether $T_{n}$ belongs to $E_{n}^{i}$ or not. Consequently, for all $i \leq k-1$, the event

$$
\left\{M_{1} \equiv \delta_{1}, \ldots, M_{i-1} \equiv \delta_{i-1}, T_{n} \in E_{n}^{i}\right\}
$$

can be written as a disjoint union (on $\mathbf{h}$ ) of events of the form $T_{n} \in \mathcal{T}_{n}^{v_{i}, \mathbf{h}}\left(t_{0}\right)$. (12) yields

$$
\mathbb{P}_{t_{0}}\left(M_{i} \equiv \delta_{i} \mid M_{1} \equiv \delta_{1}, \ldots, M_{i-1} \equiv \delta_{i-1}, T_{n} \in E_{n}^{i}\right)=\frac{1}{2}+o(1)
$$

Furthermore, for any event $A$, we have $\mathbb{P}_{t_{0}}(A)=\mathbb{P}_{t_{0}}\left(A, T_{n} \in E_{n}^{k-1}\right)+o(1)$ because $\mathbb{P}_{t_{0}}\left(T_{n} \in E_{n}^{k-1}\right) \rightarrow 1$ (Lemma 6.3)., Using this twice, we obtain

$$
\begin{aligned}
& \mathbb{P}_{t_{0}}\left(M_{1} \equiv \delta_{1}, \ldots, M_{k-1} \equiv \delta_{k-1}\right) \\
&=\mathbb{P}_{t_{0}}\left(M_{1} \equiv \delta_{1}, \ldots, M_{k-1} \equiv \delta_{k-1}, T_{n} \in E_{n}^{k-1}\right)+o(1) \\
&= \mathbb{P}_{t_{0}}\left(M_{k-1} \equiv \delta_{k-1} \mid M_{1} \equiv \delta_{1}, \ldots, M_{k-2} \equiv \delta_{k-2}, T_{n} \in E_{n}^{k-1}\right) \\
& \times\left(\mathbb{P}_{t_{0}}\left(M_{1} \equiv \delta_{1}, \ldots, M_{k-2} \equiv \delta_{k-2}\right)+o(1)\right)+o(1) \\
&= \frac{1}{2} \mathbb{P}_{t_{0}}\left(M_{1} \equiv \delta_{1}, \ldots, M_{k-2} \equiv \delta_{k-2}\right)+o(1) .
\end{aligned}
$$

Repeating this argument $k-2$ times concludes the proof.

It remains to prove that with high probability a uniform Schröder tree $T_{n}$ is well behaved: the heights $H_{i}$ of marked leaves and $M_{j}$ of their common ancestors and the distances $\left|M_{i}-M_{j}\right|$ and $\left|M_{i}-H_{j}\right|$ are all larger than $n^{1 / 4}$ (in fact, larger than $\varepsilon_{n} n^{\frac{1}{2}}$ for all $\varepsilon_{n} \rightarrow 0$ ).

LEMMA 6.3. We re-use the notation of the proof of Lemma 6.2. In particular, $E_{n}^{v}$ denotes the set of trees $t$ in $\mathcal{T}_{n}\left(t_{0}\right)$ such that $d_{t}(v) \geq n^{1 / 4}$. For all internal vertices $v$ of $t_{0}$,

$$
\mathbb{P}\left(T_{n} \notin E_{n}^{v} \mid T_{n} \in \mathcal{T}_{n}\left(t_{0}\right)\right) \rightarrow 0 .
$$

In the special case where $t_{0}=\bigvee($ i.e., $k=2)$, every pair $\left(\ell_{1}, \ell_{2}\right)$ of marked leaves of a Schröder tree with $n \geq 2$ leaves induces $t_{0}$, and the height of $\ell_{1}$ goes to infinity in probability when $n \rightarrow+\infty$.

PROOF. First, Corollary 5.4 gives the probability of the conditioning event in the limit

$$
\lim _{n \rightarrow+\infty} \mathbb{P}\left(T_{n} \in \mathcal{T}_{n}\left(t_{0}\right)\right)=\mathbb{P}\left(\operatorname{Tree}(e, \mathbf{U})=t_{0}\right)
$$


where $\mathbf{U}$ is a set of $k$ i.i.d. uniform random variables in $[0,1]$. From Lemma A.3, we know that this quantity is strictly larger than 0 . Therefore, it is enough to prove that $\mathbb{P}\left(T_{n} \notin E_{n}^{v}\right) \rightarrow 0$ without the conditioning.

By definition, for each internal vertex $v$ in $t_{0}, d_{t}(v)$ is one of the following:

- the height $H_{i}$ of a marked leaf;

- the height $M_{i}$ of the common ancestor of two marked leaves;

- the difference of heights $\left|M_{i}-M_{j}\right|$ between two common ancestors;

- the difference of heights $\left|M_{i}-H_{j}\right|$ between a leaf and a common ancestor.

Thus, the first statement of the lemma will follow if we show that

$$
\mathbb{P}\left(\begin{array}{c}
\text { There are } i \leq k, j \leq k-1 \text { such that } \\
H_{i} \leq n^{1 / 4} \text { or } M_{j} \leq n^{1 / 4} \text { or }\left|H_{i}-M_{j}\right| \leq n^{1 / 4} \\
\text { or }\left|M_{i}-M_{j}\right| \leq n^{1 / 4}(i \neq j)
\end{array}\right) \rightarrow 0 .
$$

Actually, we prove the stronger statement that for any two $i, j$, all sequences of random variables $\frac{1}{\sqrt{n}} H_{i}, \frac{1}{\sqrt{n}} M_{j}, \frac{1}{\sqrt{n}}\left|M_{i}-M_{j}\right|, \frac{1}{\sqrt{n}}\left|H_{i}-M_{j}\right|$ converge in distribution to positive random variables.

We will only write the details for the sequence $\left(\frac{1}{\sqrt{n}}\left|M_{i}-M_{j}\right|\right)$, the proof being identical in the other cases. Recall that the $x$-coordinate of $\ell_{i}$ has the same distribution as $F_{T_{n}}^{\star}\left(U_{i}\right)$, where:

- $U_{i}$ is the $i$ th smallest value among $k$ i.i.d. uniform random variables in $[0,1]$.

- $F_{T_{n}}^{\star}$ is the pseudo-inverse of $F_{T_{n}}$ defined in (4) (page 2157); combining Proposition 3.5, Lemma 3.6 (page 2157), we know that $F_{T_{n}}^{\star}(x)$ tends to $x$ when $n$ tends to infinity (uniformly in $x$ ).

By construction, we have (in what follows $\widetilde{C_{T_{n}}}$ is the normalized contour function of $T_{n}$, and the $U_{i}$ 's are independent from $\widetilde{C_{T_{n}}}$ )

$$
M_{i}=\min _{\left[F_{T_{n}}^{\star}\left(U_{i}\right), F_{T_{n}}^{\star}\left(U_{i+1}\right)\right]} \sqrt{n} \times \widetilde{C_{T_{n}}} .
$$

Since $\left(\widetilde{C_{T_{n}}}\right)_{n}$ converges to $\lambda \cdot e$ (Proposition 2.23, page 2149) and $F_{T_{n}}^{\star}\left(U_{i}\right)$ tends to $U_{i}$, we have

$$
\frac{1}{\sqrt{n}}\left|M_{i}-M_{j}\right| \stackrel{(d)}{\rightarrow} \lambda\left|\min _{\left[U_{i}, U_{i+1}\right]} e-\min _{\left[U_{j}, U_{j+1}\right]} e\right| .
$$

This completes the proof of the first statement of Lemma 6.3.

In particular, we have proved that $H_{1}$ goes to infinity with $n$ in probability. The second statement of the lemma is just a rephrasing of this, in the particular case where $t_{0}=\bigvee$.

REMARK 6.4. The joint convergence of the $\frac{1}{\sqrt{n}} H_{i}$ and $\frac{1}{\sqrt{n}} M_{i}$ is observed by Aldous in the proof of [3], Theorem 20, with the slight difference that he works 
with uniform random vertices in the tree, while we consider uniform random leaves.

ProOF OF PROPOSITION 6.1. Let $\sigma_{n}$ be a uniform separable permutation of size $n$. From Corollary 2.14 (page 2147), it has the same distribution as $\operatorname{perm}\left(T_{n}, \mathcal{E}_{n}\right)$ where $T_{n}$ is a uniform Schröder tree with $n$ leaves and $\mathcal{E}_{n}$ is the sign function on the internal vertices of $T_{n}$, such that the signs alternate and such that the root $r$ of $T_{n}$ has a balanced sign: $\mathcal{E}_{n}(r)=+$ with probability $1 / 2$. Recall that $\widetilde{C_{T_{n}}}$ [resp., $\left.\left(\widetilde{C_{T_{n}}}, S_{n}\right)\right]$ denotes the normalized contour of $T_{n}$ [resp., the signed contour of $\left.\left(T_{n}, \mathcal{E}_{n}\right)\right]$.

As in the statement of the proposition, consider a uniform random $k$-element subset $\ell$ of leaves of $T_{n}$. Recall from Observations 2.34 and 2.40 that $\operatorname{Tree}\left(\widetilde{C_{T_{n}}}, \ell\right)$ [resp., Tree $\left.\pm\left(\widetilde{C_{T_{n}}}, S_{n}, \ell\right)\right]$ is the subtree of $T_{n}$ [resp., the signed subtree of $\left(T_{n}, \mathcal{E}_{n}\right)$ ] induced by these leaves. We condition on the fact that $\operatorname{Tree}\left(\widetilde{C_{T_{n}}}, \ell\right)=t_{0}$. It is enough to prove that the probability that the signs in Tree $\pm\left(\widetilde{C_{T_{n}}}, S_{n}, \ell\right)$ coincide with a fixed sign function $\varepsilon$ on the internal vertices of $t_{0}$ is $1 / 2^{k-1}+o(1)$. Recall that the signs in Tree $\pm\left(\widetilde{C_{T_{n}}}, S_{n}, \ell\right)$ correspond to signs of the common ancestors of the marked leaves in $\left(T_{n}, \mathcal{E}_{n}\right)$.

We further condition on the fact that the root of $T_{n}$ has sign + . Then a vertex in $T_{n}$ has sign + if it has even height and sign - if it has odd height. Therefore, the probability that the common ancestors have given signs correspond to the probability that their heights have given parities. This probability is $1 / 2^{k-1}+o(1)$ by Lemma 6.2.

The same holds if we had conditioned on the fact that the root of $T_{n}$ has sign -. We can therefore conclude, that, conditioning only on the event $\operatorname{Tree}\left(\widetilde{C_{T_{n}}}, \ell\right)=t_{0}$, the probability that the common ancestors have given signs is $1 / 2^{k-1}+o(1)$, which completes the proof of the proposition.

7. Conclusion of the proof. In this section, we prove that the convergence of the expectation for $\mathrm{Pr}^{\text {Tree }}$ implies the one of the expectation for $\mathrm{Pr}^{\mathrm{Perm}}$. In order to do that, the expectation for $\operatorname{Pr}^{P e r m}$ is expressed in terms of the one for $\operatorname{Pr}^{T r e e}$ (as a linear combination) in the continuous and discrete cases. This is possible since the signs are balanced and independent as it has been proven in Proposition 6.1.

LEMMA 7.1. Let $\pi$ be a pattern of size $k$ and $(e, S)$ be the signed Brownian excursion

$$
\mathbb{E}^{e, S}\left[\operatorname{Pr}^{\operatorname{Perm}}\left(\pi ; e, S, F_{U}\right)\right]=\frac{1}{2^{k-1}} \sum_{\left(t_{0}, \varepsilon\right)} \mathbb{E}^{e}\left[\operatorname{Pr}^{\operatorname{Tree}}\left(t_{0} ; e, F_{U}\right)\right],
$$

where the sum runs over all signed binary trees $\left(t_{0}, \varepsilon\right)$ of $\pi$.

ProOF. By definition,

$$
\operatorname{Pr}^{\operatorname{Perm}}\left(\pi ; e, S, F_{U}\right)=\mathbb{P}^{\mathbf{X}}(\operatorname{Perm}(e, S, \mathbf{X})=\pi),
$$


where $\mathbf{X}$ is a set of $k$ independent uniform variables in $[0,1]$. Thus, with Observation 3.2 (page 2156),

$$
\mathbb{E}^{e, S}\left[\operatorname{Pr}{ }^{\operatorname{Perm}}\left(\pi ; e, S, F_{U}\right)\right]=\mathbb{P}^{e, S, \mathbf{X}}(\operatorname{Perm}(e, S, \mathbf{X})=\pi) .
$$

Since $\operatorname{Perm}(e, S, \mathbf{X})=\operatorname{perm}\left(\operatorname{Tree}_{ \pm}(e, S, \mathbf{X})\right)$ this is exactly the probability that Tree $_{ \pm}(e, S, \mathbf{X})$ is equal to one of the signed trees that are pre-images of $\pi$ by perm. In other terms,

$$
\mathbb{E}^{e, S}\left[\operatorname{Pr}^{\operatorname{Perm}}\left(\pi ; e, S, F_{U}\right)\right]=\sum_{\left(t_{0}, \varepsilon\right)} \mathbb{P}^{e, S, \mathbf{X}}\left(\operatorname{Tree}_{ \pm}(e, S, \mathbf{X})=\left(t_{0}, \varepsilon\right)\right),
$$

where the sum runs over all signed trees of $\pi$. From Observation 2.31 (page 2151), if $t_{0}$ is not binary, then $\operatorname{Tree}(e, S, \mathbf{X})=t_{0}$ has probability 0 , since from Lemma A.2 a.s. all minima of $e$ have distinct values. Consequently, in this case $\operatorname{Tree}_{ \pm}(e, S$, $\mathbf{X})=\left(t_{0}, \varepsilon\right)$ has also probability 0 , and the sum above can be restricted to the signed binary trees of $\pi$. For each such signed binary tree $\left(t_{0}, \varepsilon\right)$, we have

$$
\mathbb{P}^{e, S, \mathbf{X}}\left(\text { Tree }_{ \pm}(e, S, \mathbf{X})=\left(t_{0}, \varepsilon\right)\right)=\frac{1}{2^{k-1}} \mathbb{P}^{e, \mathbf{X}}\left(\operatorname{Tree}(e, \mathbf{X})=t_{0}\right)
$$

Indeed, signs are taken independently on local minima of $e$ and thus on vertices of $t_{0}$. Finally, with Observation 3.2 again, the probability on the right-hand side is

$$
\mathbb{P}^{e, \mathbf{X}}\left(\operatorname{Tree}(e, \mathbf{X})=t_{0}\right)=\mathbb{E}^{e}\left[\mathbb{P}^{\mathbf{X}}\left(\operatorname{Tree}(e, \mathbf{X})=t_{0}\right)\right]=\mathbb{E}^{e}\left[\operatorname{Pr}^{\text {Tree }}\left(t_{0} ; e, F_{U}\right)\right]
$$

which completes the proof.

Using Proposition 6.1, we obtain a discrete analogue of this lemma.

LEMMA 7.2. Let $\pi$ be a pattern of size $k$ and $\sigma_{n}$ be a uniform random separable permutation of size $n$. As usual, let $\left(\widetilde{C_{T_{n}}}, S_{n}\right)$ be the signed contour of the decomposition tree of $\boldsymbol{\sigma}_{n}$,

$$
\mathbb{E}^{\sigma_{n}}\left[\operatorname{Pr}{ }^{\operatorname{Perm}}\left(\pi ; \widetilde{C_{T_{n}}}, S_{n}, F_{T_{n}}\right)\right]=\frac{1}{2^{k-1}} \sum_{\left(t_{0}, \varepsilon\right)} \mathbb{E}^{\sigma_{n}}\left[\operatorname{Pr}^{\operatorname{Tree}}\left(t_{0} ; \widetilde{C_{T_{n}}}, F_{T_{n}}\right)\right]+o(1),
$$

where the sum runs over all signed binary trees $\left(t_{0}, \varepsilon\right)$ of $\pi$.

PROOF. Let $\mathbf{X}$ be a set of $k$ independent variables taken with distribution $F_{T_{n}}$. Remember that this amounts to choosing $k$ leaves of $T_{n}$ independently and uniformly at random. Using an argument similar to that in the previous proof, we have

$$
\left.\mathbb{E}^{\sigma_{n}}\left[\operatorname{Pr}{ }^{\operatorname{Perm}}\left(\pi ; \widetilde{C_{T_{n}}}, S_{n}, F_{T_{n}}\right)\right]=\sum_{\left(t_{0}, \varepsilon\right)} \mathbb{P}^{\sigma_{n}, \mathbf{X}_{\left(T^{\prime}\right.}}\left(\widetilde{C_{T_{n}}}, S_{n}, \mathbf{X}\right)=\left(t_{0}, \varepsilon\right)\right)
$$

where the sum runs over all signed trees $\left(t_{0}, \varepsilon\right)$ of $\pi$. 
It holds that

$$
\left.\mathbb{P}^{\sigma_{n}}, \mathbf{X}_{\left(\operatorname{Tree}_{ \pm}\right.}\left(\widetilde{C_{T_{n}}}, S_{n}, \mathbf{X}\right)=\left(t_{0}, \varepsilon\right)\right) \leq \mathbb{P}^{\sigma_{n}, \mathbf{X}}\left(\operatorname{Tree}\left(\widetilde{C_{T_{n}}}, \mathbf{X}\right)=t_{0}\right)
$$

and

$$
\begin{aligned}
\mathbb{P}^{\sigma_{n}, \mathbf{X}}\left(\operatorname{Tree}\left(\widetilde{C_{T_{n}}}, \mathbf{X}\right)=t_{0}\right) & =\mathbb{E}^{\sigma_{n}}\left[\mathbb{P}^{\mathbf{X}}\left(\operatorname{Tree}\left(\widetilde{C_{T_{n}}}, \mathbf{X}\right)=t_{0}\right)\right] \\
& =\mathbb{E}^{\sigma_{n}}\left[\operatorname{Pr}^{\operatorname{Tree}}\left(t_{0} ; \widetilde{C_{T_{n}}}, F_{T_{n}}\right)\right] .
\end{aligned}
$$

If $t_{0}$ is not binary, using Corollary 5.4 (page 2168), we further have

$$
\mathbb{E}^{\sigma_{n}}\left[\operatorname{Pr}^{\operatorname{Tree}}\left(t_{0} ; \widetilde{C_{T_{n}}}, F_{T_{n}}\right)\right] \rightarrow \mathbb{E}^{e}\left[\operatorname{Pr}^{\operatorname{Tree}}\left(t_{0} ; e, F_{U}\right)\right]=0 .
$$

Recall indeed that, since a.s. all minima of $e$ have distinct values from Lemma A.2, the trees extracted from $e$ at uniformly distributed points are binary with probability 1 .

Therefore, the sum in (14) can be replaced by a sum over signed binary trees $\left(t_{0}, \varepsilon\right)$ of $\pi$, making only an error of $o(1)$. For signed binary trees $\left(t_{0}, \varepsilon\right)$, we use Proposition 6.1 (page 2168) to derive that

$$
\begin{aligned}
\mathbb{P}^{\sigma_{n}, \mathbf{X}} & \left(\operatorname{Tree}_{ \pm}\left(\widetilde{C_{T_{n}}}, S_{n}, \mathbf{X}\right)=\left(t_{0}, \varepsilon\right)\right) \\
& \left.=\mathbb{P}^{\sigma_{n}}, \mathbf{X}_{\left(\operatorname{Tree}_{ \pm}\right.}\left(\widetilde{C_{T_{n}}}, S_{n}, \mathbf{X}\right)=\left(t_{0}, \varepsilon\right) \mid \text { no repetition in } \mathbf{X}\right)+o(1) \\
& =\frac{1}{2^{k-1}} \mathbb{P}^{\sigma_{n}, \mathbf{X}}\left(\operatorname{Tree}\left(\widetilde{C_{T_{n}}}, \mathbf{X}\right)=t_{0} \mid \text { no repetition in } \mathbf{X}\right)+o(1) \\
& =\frac{1}{2^{k-1}} \mathbb{P}^{\sigma_{n}, \mathbf{X}}\left(\operatorname{Tree}\left(\widetilde{C_{T_{n}}}, \mathbf{X}\right)=t_{0}\right)+o(1) .
\end{aligned}
$$

Finally, since

$$
\begin{aligned}
& \mathbb{P}^{\sigma_{n}}, \mathbf{X}\left(\operatorname{Tree}\left(\widetilde{C_{T_{n}}}, \mathbf{X}\right)=t_{0}\right)=\mathbb{E}^{\sigma_{n}}\left[\mathbb{P}^{\mathbf{X}}\left(\operatorname{Tree}\left(\widetilde{C_{T_{n}}}, \mathbf{X}\right)=t_{0}\right)\right] \\
& =\mathbb{E}^{\sigma_{n}}\left[\operatorname{Pr}^{\operatorname{Tree}}\left(t_{0} ; \widetilde{C_{T_{n}}}, F_{T_{n}}\right)\right],
\end{aligned}
$$

this completes the proof.

Using these expressions of the expectation for $\operatorname{Pr}^{P e r m}$ in terms of the one for $\operatorname{Pr}^{T r e e}$ in the continuous and discrete cases, we can now prove our main result.

THEOREM 7.3. For any pattern $\pi$,

$$
\mathbb{E}\left[\widetilde{\operatorname{occ}}\left(\pi, \sigma_{n}\right)\right] \longrightarrow \mathbb{E}\left[\Lambda_{\pi}\right]
$$

PROOF. From Corollary 5.4, we have that, for each tree $t_{0}$,

$$
\mathbb{E}^{\sigma_{n}}\left[\operatorname{Pr}^{\text {Tree }}\left(t_{0} ; \widetilde{C_{T_{n}}}, F_{T_{n}}\right)\right] \rightarrow \mathbb{E}^{e}\left[\operatorname{Pr}^{\text {Tree }}\left(t_{0} ; e, F_{U}\right)\right]
$$

Combining this with Lemmas 7.1 and 7.2, we get that for each pattern $\pi$,

$$
\mathbb{E}^{\sigma_{n}}\left[\operatorname{Pr}^{\operatorname{Perm}}\left(\pi ; \widetilde{C_{T_{n}}}, S_{n}, F_{T_{n}}\right)\right] \rightarrow \mathbb{E}^{e, S}\left[\operatorname{Pr}^{\operatorname{Perm}}\left(\pi ; e, S, F_{U}\right)\right]
$$


From Corollary 3.11 (page 2160), $\widetilde{\mathrm{occ}}\left(\pi, \sigma_{n}\right)=\operatorname{Pr}^{\mathrm{Perm}}\left(\pi ; \widetilde{C_{T_{n}}}, S_{n}, F_{T_{n}}\right)(1+$ $\left.\mathcal{O}\left(\frac{1}{n}\right)\right)$. Together with Observation 3.9 (stating that $\Lambda_{\pi}=\operatorname{Pr}^{\operatorname{Perm}}\left(\pi ; e, S, F_{U}\right)$ ), this completes the proof of Theorem 7.3.

As shown by Corollary 4.6 (page 2165), Theorems 4.1 and 1.2(iii) follow from Theorem 7.3 above.

8. Permuton interpretation of our main result. The goal of this section is to prove Theorem 1.6 (page 2141). We first need additional material on permutons. Recall from Definition 1.5 that permutons, which were introduced in [23], are probability measures on $[0,1]^{2}$ with uniform marginals. Given a (deterministic) permuton $\mu$ and an integer $k$, there is a natural way to define a random permutation $\Pi_{k}^{\mu}$ of size $k$.

DEFINITION 8.1. Let $\mu$ be a permuton and $k$ be an integer. Take $k$ points in $[0,1]^{2}$ independently according to $\mu$. A.s. these $k$ points have distinct $x$ coordinates and distinct $y$-coordinates (since $\mu$ has uniform marginals). Therefore, we can order these points $\left(X_{1}, Y_{1}\right), \ldots,\left(X_{k}, Y_{k}\right)$ such that $Y_{1}<Y_{2}<\cdots<Y_{k}$. Then $\Pi_{k}^{\mu}$ is the unique permutation such that $X_{\Pi_{k}^{\mu}(1)}<X_{\Pi_{k}^{\mu}(2)}<\cdots<X_{\Pi_{k}^{\mu}(k)}$.

For a permutation $\pi$ of size $k$, following the notation of [23], we then define $t(\pi, \mu)$ as the probability that $\Pi_{k}^{\mu}=\pi$.

We say that a (deterministic) sequence of permutations $\left(\sigma_{n}\right)$ with sizes going to infinity converges to the permuton $\mu$ if, for all $\pi, \widetilde{\text { occ }}\left(\pi, \sigma_{n}\right)$ tends to $t(\pi, \mu)$.

It is noticed in [23], equation (49), that the convergence of $\left(\sigma_{n}\right)$ to a permuton $\mu$ is equivalent to the weak convergence of the associated permutons $\left(\mu_{\sigma_{n}}\right)$ to $\mu$. In particular, this implies the uniqueness of limits of sequences of permutations. Moreover, from [23], Theorem 1.6(i), if $\left(\sigma_{n}\right)$ is a deterministic sequence of permutations such that $\widetilde{\text { occ }}\left(\pi, \sigma_{n}\right)$ has a limit for all $\pi$, then there exists a (necessarily unique) permuton $\mu$ such that $\left(\sigma_{n}\right)$ tends towards $\mu$.

We can now proceed to the proof of Theorem 1.6.

Proof of TheOREM 1.6. Item (iii) in Theorem 1.2 asserts that the finitedimensional laws of $\left(\widetilde{\mathrm{occ}}\left(\pi, \sigma_{n}\right)\right)_{\pi}$ converge to those of $\left(\Lambda_{\pi}\right)_{\pi}$ (here, vectors are indexed by all permutations).

This is equivalent to the convergence in distribution of the infinite-dimensional vector $\left(\widetilde{\mathrm{occ}}\left(\pi, \sigma_{n}\right)\right)_{\pi}$ towards $\left(\Lambda_{\pi}\right)_{\pi}$ in the product topology (from the definitions of the convergence in distribution and of the product topology). From Skorohod's representation theorem [26], Theorem 3.30, there exists a probability space $\Omega$, random variables $\left(\boldsymbol{O}_{n}\right)_{n \geq 1}$ and $\boldsymbol{\Lambda}^{\prime}$ such that:

(i) for each $n \geq 1, \boldsymbol{O}_{n}$ is a random vector $\left(O_{n, \pi}\right)_{\pi}$ indexed by all permutations that has the same law as $\left(\widetilde{\mathrm{occ}}\left(\pi, \sigma_{n}\right)\right)_{\pi}$; 
(ii) $\Lambda^{\prime}$ is a random vector $\left(\Lambda_{\pi}^{\prime}\right)_{\pi}$ indexed by all permutations that has the same law as $\left(\Lambda_{\pi}\right)_{\pi}$;

(iii) we have sure convergence (in the product topology) of $\boldsymbol{O}_{n}$ to $\boldsymbol{\Lambda}^{\prime}$ when $n$ tends to infinity, that is, for any $\omega \in \Omega$, we have

$$
\text { for any permutation } \pi, \quad O_{n, \pi}(\omega) \rightarrow \Lambda^{\prime}(\omega)_{\pi} \text {. }
$$

We now aim at constructing random variables $\boldsymbol{\sigma}_{n}^{\prime}$ such that

$$
\left(O_{n, \pi}\right)_{\pi}=\left(\widetilde{\mathrm{occ}}\left(\pi, \sigma_{n}^{\prime}\right)\right)_{\pi},
$$

so that we can apply Theorem 1.6(i) of [23] in each $\omega \in \Omega$.

Fix $n \geq 1$. Since $\left(O_{n, \pi}\right)_{\pi}$ and $\left(\widetilde{\mathrm{occ}}\left(\pi, \sigma_{n}\right)\right)_{\pi}$ have the same distribution and since this distribution is supported on a finite set, one can assume that they have the same image set. Thus, for any $\omega$ in $\Omega,\left(O_{n, \pi}(\omega)\right)_{\pi}$ is equal to $(\widetilde{\mathrm{occ}}(\pi, \sigma))_{\pi}$ for some permutation $\sigma$ of size $n$ that we denote $\sigma_{n}^{\prime}(\omega)$. This defines a sequence of random permutations $\sigma_{n}^{\prime}$ defined on the probability space $\Omega$ with the property that $\left(O_{n, \pi}\right)_{\pi}=\left(\widetilde{\mathrm{occ}}\left(\pi, \sigma_{n}^{\prime}\right)\right)_{\pi}$. Note that $\sigma_{n}^{\prime}$ is measurable since, for a permutation $\tau$ of size $n$, we have $\left\{\omega: \sigma_{n}^{\prime}(\omega)=\tau\right\}=\left\{\omega: O_{n, \tau}(\omega)=1\right\}$.

Next, we claim that for any fixed $n, \sigma_{n}^{\prime}$ has the same distribution as $\sigma_{n}$. Indeed, $n$ being fixed, considering the restriction of $\left(\widetilde{\mathrm{occ}}\left(\pi, \sigma_{n}\right)\right)_{\pi}\left[\operatorname{resp} .,\left(\widetilde{\mathrm{occ}}\left(\pi, \sigma_{n}^{\prime}\right)\right)_{\pi}\right]$ to patterns $\pi$ of size $n$ gives the distribution of $\sigma_{n}$ (resp., $\boldsymbol{\sigma}_{n}^{\prime}$ ). The claim then follows since $\left(\widetilde{\mathrm{occ}}\left(\pi, \sigma_{n}\right)\right)_{\pi}$ and $\left(\widetilde{\mathrm{occ}}\left(\pi, \sigma_{n}^{\prime}\right)\right)_{\pi}$ have the same distribution (both the same as that of $\left.\left(O_{n, \pi}\right)_{\pi}\right)$.

From Item (iii) above, and by definition of $\boldsymbol{\sigma}_{n}^{\prime}$, for any $\omega \in \Omega$, the following holds:

$$
\text { for any permutation } \pi, \quad \widetilde{\mathrm{occ}}\left(\pi, \sigma_{n}^{\prime}(\omega)\right) \rightarrow \Lambda^{\prime}(\omega)_{\pi} .
$$

Using [23], Theorem 1.6(i), this implies that, for any $\omega \in \Omega$, there exists a (unique) permuton $\boldsymbol{\mu}(\omega)$ such that $\boldsymbol{\sigma}_{n}^{\prime}(\omega)$ tends to $\boldsymbol{\mu}(\omega)$ in the sense of Definition 8.1 , or equivalently $\mu_{\sigma_{n}^{\prime}(\omega)}$ converges weakly to $\boldsymbol{\mu}(\omega)$. Since $\omega \mapsto \boldsymbol{\mu}(\omega)$ is the pointwise limit of the measurable functions $\omega \mapsto \mu_{\sigma_{n}^{\prime}(\omega)}$ in the metrizable weak topology, we know that $\boldsymbol{\mu}$ is measurable.

Using that pointwise convergence implies convergence in distribution and that $\boldsymbol{\sigma}_{n}^{\prime}$ and $\boldsymbol{\sigma}_{n}$ have the same distribution, we deduce that $\mu_{\boldsymbol{\sigma}_{n}}$ tends in distribution to $\boldsymbol{\mu}$ in the weak convergence topology, as claimed.

Finally, it should be noted that, for any $\pi, \Lambda_{\pi} \stackrel{(d)}{=} t(\pi, \mu)$, using the notation $t$ from Definition 8.1, since both are the limit in distribution of $\widetilde{\text { occ }}\left(\pi, \sigma_{n}\right)$. From Theorem 1.2(i) $\Lambda_{12}$ is not deterministic, so neither is $\boldsymbol{\mu}$.

9. Some properties of $\boldsymbol{\Lambda}_{\pi}$. In this section we give some additional results concerning the limit variables $\Lambda_{\pi}$. These results are not needed in the proof of our main theorem [Theorem 1.2(iii)]. However, the more information we have on $\Lambda_{\pi}$, the more interesting our theorem is. 
The first result, given in Section 9.1, is a way of computing (joint) moments of the variables $\Lambda_{\pi}$. This has been used in the Introduction to give explicit values for the limit of (joint) moments of $\widetilde{\mathrm{occ}}\left(\pi, \sigma_{n}\right)$. Our second result presented in Section 9.2 is the fact that the variables $\Lambda_{\pi}$ are not deterministic when $\pi$ is separable. This corresponds to Item (i) in Theorem 1.2.

9.1. Computing expectation and other moments of $\Lambda_{\pi}$. Recall that $N_{\pi}$ denotes the number of signed binary trees associated with the permutation $\pi$.

Proposition 9.1. For any permutation $\pi$ of size $k$,

$$
\mathbb{E}\left[\widetilde{\mathrm{occ}}\left(\pi, \sigma_{n}\right)\right] \longrightarrow \mathbb{E}\left[\Lambda_{\pi}\right]=\frac{N_{\pi}}{2^{k-1} \cdot \mathrm{Cat}_{k-1}}
$$

PROOF. For any $\pi$, the convergence of $\mathbb{E}\left[\widetilde{\mathrm{occ}}\left(\pi, \sigma_{n}\right)\right]$ to $\mathbb{E}\left[\Lambda_{\pi}\right]$ is given by Theorem 7.3 (page 2176). Let $\pi$ be a permutation of size $k$ and let us prove that

$$
\mathbb{E}\left[\Lambda_{\pi}\right]=\frac{N_{\pi}}{2^{k-1} \cdot \mathrm{Cat}_{k-1}} .
$$

We consider the signed Brownian excursion $(e, S)$. We take $k$ points $X_{1}, \ldots, X_{k}$ in $[0,1]$ uniformly at random, independently from each other and from $(e, S)$, and we let $\mathbf{X}=\left(X_{1}, \ldots, X_{k}\right)$. From Lemma 7.1 (see also (13) in its proof), we have

$$
\mathbb{E}^{e, S}\left[\operatorname{Pr}^{\mathrm{Perm}}\left(\pi ; e, S, F_{U}\right)\right]=\frac{1}{2^{k-1}} \sum_{\left(t_{0}, \varepsilon\right)} \mathbb{P}^{e, \mathbf{X}}\left(\operatorname{Tree}(e, \mathbf{X})=t_{0}\right),
$$

where the sum runs over all signed binary trees $\left(t_{0}, \varepsilon\right)$ of $\pi$.

Lemma A.3, a deep result on Brownian excursion, states that $\operatorname{Tree}(e, \mathbf{X})$ is a uniform binary tree with $k$ leaves, that is, for any binary tree $t_{0}$ with $k$ leaves,

$$
\mathbb{P}^{e, \mathbf{X}}\left(\operatorname{Tree}(e, \mathbf{X})=t_{0}\right)=\frac{1}{\mathrm{Cat}_{k-1}} .
$$

The proposition then follows immediately.

In addition, $N_{\pi}$, the number of signed binary trees of a given permutation $\pi$, can be expressed combinatorially from the decomposition tree of $\pi$. More precisely, we have the following.

OBSERVATION 9.2. For any permutation $\pi$, denoting $t_{\pi}$ its (unique) decomposition tree, it holds that

$$
N_{\pi}:=\operatorname{card}\left\{\left(t_{0}, \varepsilon\right), t_{0} \text { binary }: \operatorname{perm}\left(t_{0}, \varepsilon\right)=\pi\right\}=\prod_{v \text { int. vertex of } t_{\pi}} \operatorname{Cat}_{\operatorname{deg}(v)-1},
$$

where $\operatorname{deg}(v)$ is the number of children of $v$. 

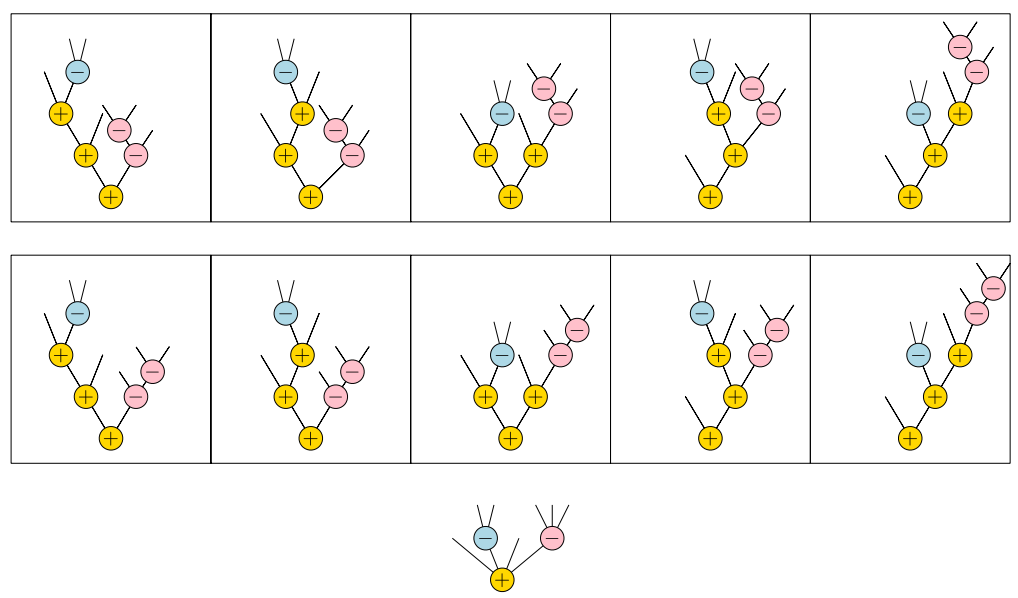

FIG. 12. The decomposition tree of 1324765 and its $\mathrm{Cat}_{3} \times \mathrm{Cat}_{2} \times \mathrm{Cat}_{1}=10$ signed binary trees.

Proof. Given a signed tree of $\pi$, and a vertex $v$ with sign $\varepsilon$ in this tree, the following transformation produces a tree which is still a signed tree of $\pi$ : assuming that $k$ subtrees $T_{1}, \ldots, T_{k}$ are attached to $v$, replace $v$ by a binary tree with $k$ leaves and all internal vertices labeled $\varepsilon$ and where the $i$ th leaf is replaced by $T_{i}$. Conversely, each signed binary tree of $\pi$ can be obtained from the decomposition tree $t_{\pi}$ of $\pi$ applying this transformation on all internal vertices $v$ of $t_{\pi}$. An example is given in Figure 12.

PROPOSITION 9.3. Let $\pi_{1}, \ldots, \pi_{r}$ be a list of patterns, and let $K=\sum_{i=1}^{r}\left|\pi_{i}\right|$. We have

$$
\mathbb{E}\left[\prod_{i=1}^{r} \widetilde{\operatorname{occ}}\left(\pi_{i}, \sigma_{n}\right)\right] \longrightarrow \mathbb{E}\left[\prod_{i=1}^{r} \Lambda_{\pi_{i}}\right]=\sum_{\rho \in \mathfrak{S}_{K}} c_{\pi_{1}, \ldots, \pi_{r}}^{\rho} \mathbb{E}\left[\Lambda_{\rho}\right],
$$

where for any $\rho \in \mathfrak{S}_{K}, c_{\pi_{1}, \ldots, \pi_{r}}^{\rho}$ denotes the proportion of ordered set-partitions of $\{1, \ldots, K\}$ which are compatible with $\rho, \pi_{1}, \ldots, \pi_{r}$; see Definition 4.2 , page 2161.

PROOF. See the proof of Corollary 4.6 for the convergence result, and Proposition 4.4 for the expression of $\mathbb{E}\left[\Pi \Lambda_{\pi_{i}}\right]$.

The results of this section enable the automatic computation of joint moments of $\Lambda_{\pi}$. As mentioned in the Introduction, we have implemented this in Sage. Let us discuss quickly algorithmic questions behind this implementation:

- From Proposition 9.1, computing the expectation essentially amounts to computing $N_{\pi}$. Finding the degree of the root of the decomposition tree of $\pi$ is easy. To simplify the discussion (but without loss of generality), suppose that this root 
has sign + . Then its degree is the number of integers $i \leq k$ such that

$$
\{\pi(1), \ldots, \pi(i)\}=\{1, \ldots, i\}, \quad \text { or equivalently } \quad \max _{j \leq i} \pi(j)=i .
$$

Thanks to the second formulation, this can be computed in linear time reading the permutation from left-to-right, keeping only in memory the maximum of the values already read.

Degrees of all vertices can then be computed recursively, and we find $N_{\pi}$ using Observation 9.2, in quadratic time.

- Higher moments or joint moments are more complex to compute. Fix a list of patterns $\pi_{1}, \ldots, \pi_{r}$ of respective sizes $k_{1}, \ldots, k_{r}$ and set $K=k_{1}+\cdots+k_{r}$. While convenient in proofs, the definition of the coefficients $c_{\pi_{1}, \ldots, \pi_{r}}^{\rho}$ (see Definition 4.2, page 2161) is not optimal from a practical point of view. It is however possible to generate directly the multi-set of permutations of $\mathfrak{S}_{K}$, where each $\rho$ appears with multiplicity $d_{\pi_{1}, \ldots, \pi_{r}}^{\rho}$. If Pos $=\left(\operatorname{Pos}_{1}, \ldots, \operatorname{Pos}_{r}\right)$ and $\mathbf{V a l}=\left(\mathrm{Val}_{1}, \ldots, \mathrm{Val}_{r}\right)$ are ordered set-partitions of $\{1, \ldots, K\}$ such that $\operatorname{card}\left(\operatorname{Pos}_{i}\right)=\operatorname{card}\left(\operatorname{Val}_{i}\right)=k_{i}$, we can define a permutation $\rho(\mathbf{P o s}$, Val $)$ as follows: $\rho$ (Pos, Val) assigns the $\pi_{i}(j)$ th smallest value in $\mathrm{Val}_{i}$ to the $j$ th smallest value in $\operatorname{Pos}_{i}$. It is easy to check that any given $\rho$ is constructed this way from exactly $d_{\pi_{1}, \ldots, \pi_{r}}^{\rho}$ pairs (Pos, Val). We have used this construction in our implementation of the computation of joint moments.

Even if this is more efficient than a naive implementation of Definition 4.2, the complexity still grows very quickly.

Indeed, the number of pairs (Pos, Val) as above is equal to $\left(\begin{array}{c}K \\ k_{1}, \ldots, k_{r}\end{array}\right)^{2}$. In practice, we have only been able to compute the first four moments of $\Lambda_{12}$ with this algorithm (the fifth moment given in the Introduction has been inferred from the first four using the symmetry $\left.\Lambda_{12} \stackrel{(d)}{=} 1-\Lambda_{12}\right)$. We believe that modifying slightly the program to enable parallel computing may allow to compute the fifth moment with the above algorithm in a reasonable time but that the sixth moment, which involves more than $5 \times 10^{13}$ pairs $(\mathbf{P o s}$, Val), will remain out of reach.

9.2. $\Lambda_{\pi}$ is not deterministic. Finally, we will prove Theorem 1.2(i) We have already seen in Remark 3.3 (page 2156) that, when $\pi$ is not separable, $\Lambda_{\pi}$ is identically 0 (in particular, deterministic). We have also seen in Remark 3.4 (page 2156) that for the permutation $\pi$ of size $1, \Lambda_{\pi}$ is identically 1 (and thus, again deterministic). On the contrary, we now prove that if $\pi$ is separable of size at least 2 , then $\Lambda_{\pi}$ is a true random variable and not a deterministic constant.

When $\pi$ is separable of size at least 2 , to prove that $\Lambda_{\pi}$ is not deterministic, it would be sufficient to check that $\operatorname{Var}\left(\Lambda_{\pi}\right)>0$. Although Proposition 9.3 provides an expression of the variance of $\Lambda_{\pi}$, this expression does not allow us to prove directly that $\operatorname{Var}\left(\Lambda_{\pi}\right)>0$. Instead, we prove in Proposition 9.5 that $\Lambda_{\pi} \neq \mathbb{E}\left[\Lambda_{\pi}\right]$ with positive probability. From the expression of $\mathbb{E}\left[\Lambda_{\pi}\right]$ given in Proposition 9.1, 


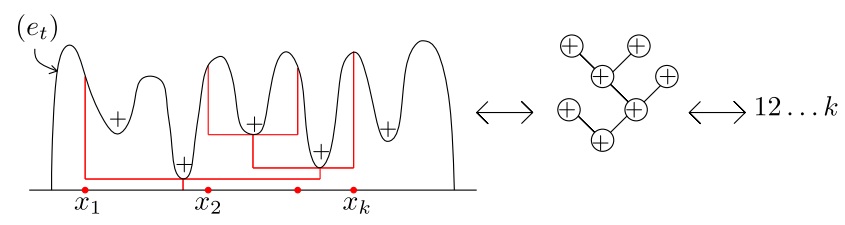

FIG. 13. A realization of $(e, S)$ for which $\operatorname{Perm}(e, S, \mathbf{X})=123 \ldots k$ with high probability.

it follows immediately that $\mathbb{E}\left[\Lambda_{\pi}\right]>0$ if $\pi$ is separable. So the proof of Theorem 1.2(i) will be completed as soon as we prove that $\Lambda_{\pi}$ takes values as close to 0 as wanted with positive probability. This is done in Lemma 9.4.

LEMMA 9.4. Let $\pi$ be a separable permutation of size $k \geq 2,(e, S)$ be the signed Brownian excursion and $N$ be a positive integer. There exists an event $E_{N}=$ $E_{N}(e, S)$ such that:

- $E_{N}$ occurs with positive probability;

- If $(e, S)$ is such that $E_{N}$ occurs, then $\mathbb{P}^{\mathbf{X}_{(}}(\operatorname{Perm}(e, S, \mathbf{X})=\pi) \leq \frac{k^{2}}{N}$, where $\mathbf{X}=$ $\left(X_{1}, \ldots, X_{k}\right)$ and the $X_{i}$ 's are uniform independent points in $[0,1]$.

Proof. Assume first that $\pi \neq 123 \ldots k$. The key idea of the proof is that for some realizations of $(e, S)$ (see a sketch in Figure 13), Perm $(e, S, \mathbf{X})=123 \ldots k$ with high probability.

We set $\eta=\frac{1}{4 N^{2}}$ and define intervals as follows:

- for integers $\ell$ between 0 and $N$, we set $J_{\ell}=\left[\frac{\ell}{N}-\eta ; \frac{\ell}{N}+\eta\right] \cap[0,1]$;

- for integers $\ell$ between 1 and $N$, we set $I_{\ell}=\left[\frac{\ell-1}{N}+\eta ; \frac{\ell}{N}-\eta\right]$.

These intervals are represented on Figure 14. We have Length $\left(I_{\ell}\right)=1 / N-2 \eta$ for each $\ell$. Moreover, by construction Length $\left(J_{\ell}\right)=2 \eta$ for $\ell \neq 0$ and $\ell \neq N$, while Length $\left(J_{0}\right)=\operatorname{Length}\left(J_{N}\right)=\eta$.

Let $E_{N}=E_{N}(e, S)$ be the event defined as follows:

(i) For each $\ell, \min _{J_{\ell}} e \leq 1 / 10$.

(ii) $\min _{I_{1} \cup I_{2} \cup \ldots \cup I_{N}} e \geq 2 / 10$.

(iii) The signs of the $N-1$ local minima of $e$ on $J_{1}, \ldots, J_{N-1}$ are all + .

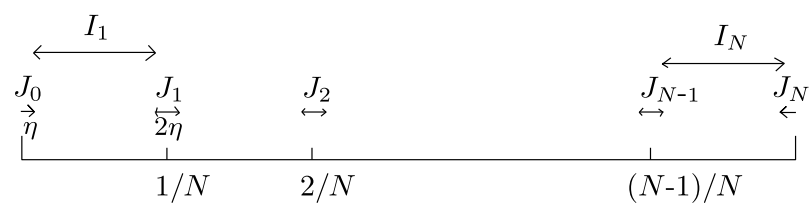

FIG. 14. Intervals $I_{\ell}$ and $J_{\ell}$. 
Taking $f$ a piecewise linear excursion such that $f\left(\frac{\ell}{N}\right)=0$ for integers $\ell$ between 0 and $N$ and $f(x)=3 / 10$ for $x \in I_{1} \cup I_{2} \cup \cdots \cup I_{N}$, and with $\delta=1 / 10$, it follows from Lemma A.5 that

$$
\mathbb{P}^{e, S}\left(E_{N}\right)=\frac{1}{2^{N-1}} \mathbb{P}^{e}(e \text { satisfies Items (i) and (ii) })>0 .
$$

Consider now a realization $(e, S)$ of the signed Brownian excursion such that $E_{N}$ is realized, and let $\mathbf{X}=\left(X_{1}, \ldots, X_{k}\right)$ be $k$ uniform independent points in [0,1]. The construction of $E_{N}$ ensures that $\operatorname{Perm}(e, S, \mathbf{X})=123 \ldots k$ as soon as each $X_{i}$ is in an interval $I_{\ell}$ such that $I_{\ell}$ does not contain any other $X_{j}$. Equivalently, if $\operatorname{Perm}(e, S, \mathbf{X}) \neq 123 \ldots k$ then one of the two following events occurs:

- For some $i, X_{i}$ belongs to an interval $J_{\ell}$.

- For some $i \neq j, X_{i}$ and $X_{j}$ belong to the same interval $I_{\ell}$.

Therefore, we get

$$
\begin{aligned}
\mathbb{P}^{\mathbf{X}}(\operatorname{Perm}(e, S, \mathbf{X})=\pi) & \leq \mathbb{P}^{\mathbf{X}}(\operatorname{Perm}(e, S, \mathbf{X}) \neq 123 \ldots k) \\
& \leq k \sum_{\ell=0}^{N} \mathbb{P}^{\mathbf{X}}\left(X_{1} \in J_{\ell}\right)+\left(\begin{array}{l}
k \\
2
\end{array}\right) \sum_{\ell=1}^{N} \mathbb{P}^{\mathbf{X}}\left(X_{1}, X_{2} \in I_{\ell}\right) \\
& \leq k 2 N \eta+\left(\begin{array}{l}
k \\
2
\end{array}\right) N\left(\frac{1}{N}-2 \eta\right)^{2} \\
& \leq \frac{k}{2 N}+\left(\begin{array}{l}
k \\
2
\end{array}\right) \frac{1}{N}=\frac{k^{2}}{N} \quad\left(\text { recall that } \eta=\frac{1}{4 N^{2}}\right) .
\end{aligned}
$$

This completes the proof of Lemma 9.4 when $\pi \neq 123 \ldots k$. By symmetry, the same result holds for $\pi \neq k \ldots 321$. Therefore, the statement of Lemma 9.4 holds for all separable permutations of size at least 2.

We can now establish the announced proposition.

PROPOSITION 9.5. For any separable pattern $\pi$ of size at least 2,

$$
\mathbb{P}^{e, S}\left(\Lambda_{\pi}<\mathbb{E}\left[\Lambda_{\pi}\right]\right)>0 .
$$

In particular, $\Lambda_{\pi}$ is not almost surely constant.

PROOF. Let $k \geq 2$ be the size of $\pi$. Since $\mathbb{E}\left[\Lambda_{\pi}\right]>0$ (from Proposition 9.1) and $k$ is fixed, one can choose $N$ big enough such that $k^{2} / N<\mathbb{E}\left[\Lambda_{\pi}\right]$. For such a value of $N$, let $E_{N}$ be the event given by Lemma 9.4. If $(e, S)$ is such that $E_{N}$ is realized, then (with $\mathbf{X}=\left(X_{1}, \ldots, X_{k}\right)$ a tuple of $k$ uniform independent points in $[0,1])$

$$
\Lambda_{\pi}=\mathbb{P}^{\mathbf{X}}(\operatorname{Perm}(e, S, \mathbf{X})=\pi) \leq \frac{k^{2}}{N}<\mathbb{E}\left[\Lambda_{\pi}\right]
$$


Since $E_{N}$ has positive probability, the event $\Lambda_{\pi}<\mathbb{E}\left[\Lambda_{\pi}\right]$ also occurs with positive probability.

\section{APPENDIX: USEFUL FACTS ON THE BROWNIAN EXCURSION}

For the convenience of the reader, we now record several useful properties of a typical realization of the Brownian excursion $e$.

There are several ways to define the Brownian excursion, the most convenient for us is to draw a realization of $e$ from a realization of the Brownian motion $\left(B_{t}\right)_{t \geq 0}$, as follows (see [36], Section 0.2). Consider

$$
a=\sup \left\{t \leq 1: B_{t}=0\right\}, \quad b=\inf \left\{t \geq 1: B_{t}=0\right\}
$$

(almost surely $a<1<b$ ), and set

$$
(e(s))_{0 \leq s \leq 1}:=\left(\frac{1}{\sqrt{b-a}}\left|B_{a+s(b-a)}\right|\right)_{0 \leq s \leq 1} .
$$

Thus, the Brownian excursion $e$ is seen as a dilatation of a piece of $B$. It follows that some almost-sure properties of the set of local extrema of $B$ remain true for $e$.

Recall that by definition, $x$ is a one-sided local minimum for $f$ if, for some $\varepsilon>0$,

$$
f(x)=\min _{[x-\varepsilon, x]} f \quad \text { or } \quad f(x)=\min _{[x, x+\varepsilon]} f .
$$

LEMMA A.1. The set of one-sided local minima of the Brownian excursion e has Lebesgue measure 0, almost surely.

PROOF. We first prove an analogous statement for the Brownian motion $\left(B_{t}\right)_{t \geq 0}$. We denote $\operatorname{Omin}(B)$ the set of one-sided local minima of $B$, and $\operatorname{Leb}(\mathrm{Omin}(B))$ its Lebesgue measure. We have

$$
\operatorname{Leb}(\operatorname{Omin}(B))=\int_{0}^{+\infty} \mathbf{1}_{u \in \operatorname{Omin}(B)} d u,
$$

so that, taking the expectation with respect to $B$,

$$
\mathbb{E}[\operatorname{Leb}(\operatorname{Omin}(B))]=\int_{0}^{+\infty} \mathbb{P}(u \in \operatorname{Omin}(B)) d u=0 .
$$

In the last equality, we used that $\mathbb{P}(u \in \operatorname{Omin}(B))=0$ for every fixed $u$ : Theorem 1.27 in [35] gives a similar result for local minima (i.e., two-sided minima), but the proof is easily adapted to the case of one-sided minima. Thus, $\operatorname{Leb}(\operatorname{Omin}(B))$ is a nonnegative random variable with expectation 0 and, therefore, is equal to 0 almost surely.

The statement then follows for $\operatorname{Leb}(\operatorname{Omin}(e))$ by dilatation, since the dilatation of a set of measure zero has measure zero as well.

We now discuss values of local minima. 
LEMMA A.2. With probability one, the Brownian excursion e has no two local minima with the same value.

PROOF. For the Brownian motion, it is the statement of [26], Lemma 11.15. This is also true for $e$ since it is a dilatation of the Brownian motion.

An important consequence of Lemma A.2 in the present paper is that, for all set of $k$ distinct points $\mathbf{x}$ of $[0,1]$ and for almost all realizations of $e$, the tree Tree $(e, \mathbf{x})$ obtained in Section 2.4 is a binary tree (because of Observation 2.31).

A remarkable fact is that if $\mathbf{x}$ is uniformly distributed this random binary tree is uniform (see [30], Section 2.6).

LEMMA A.3. Fix $k \geq 2$ and a binary tree $t_{0}$ with $k$ leaves. Let $U_{1}, \ldots, U_{k}$ be $k$ uniform and independent random variables in $[0,1]$, independent from $e$. Then

$$
\mathbb{P}\left(\text { Tree }\left(e,\left\{U_{1}, \ldots, U_{k}\right\}\right)=t_{0}\right)=\frac{1}{\mathrm{Cat}_{k-1}} .
$$

It is in fact even possible to describe the law of the geometric tree extracted from $e$ and $U_{1}, \ldots, U_{k}$, that is, a tree with edge-lengths that are nonnegative real numbers (see [30], Theorem 2.11). In this paper, we use a rather weak consequence of this result.

LEMMA A.4. Take $k$ i.i.d. uniform random variables in $[0,1]$ independently from $e$ and call them $U_{1}<\cdots<U_{k}$. Set $M_{i}=\min _{\left[U_{i}, U_{i+1}\right]}$. Then the random vector

$$
\mathbf{v}=\left(e\left(U_{1}\right), \ldots, e\left(U_{k}\right), M_{1}, \ldots, M_{k-1}\right)
$$

has distinct coordinates with probability 1.

ProOF. As said above, the law of the geometric tree extracted from $e$ and $U_{1}, \ldots, U_{k}$ is given in [30], Theorem 2.11 ; this law has a density with respect to the uniform distribution on geometric trees.

Conditioning on the fact that $\operatorname{Tree}\left(e,\left\{U_{1}, \ldots, U_{k}\right\}\right)$ is a given $t_{0}$ with $k$ leaves, the coordinates of $\mathbf{v}$ are sums of edge-lengths of the geometric tree. Hence, the vector $\mathbf{v}$ has a density with respect to the Lebesgue measure on $\mathbb{R}^{2 k-1}$. Without conditioning, $\mathbf{v}$ has also a density, which is simply the mean of the conditional densities. This implies the lemma.

Finally, we need the fact that the Brownian excursion $e$ is arbitrary close to any fixed Lipschitz excursion with positive probability. (A Lipschitz excursion is simply an excursion that is also a Lipschitz function, that is, there exists $C>0$ such that $|f(x)-f(y)| \leq C|x-y|$ for all $x, y$ in $[0,1]$.) 
LEMMA A.5. For every Lipschitz excursion $f$ and $\delta>0$,

$$
\mathbb{P}\left(\sup _{0 \leq s \leq 1}|e(s)-f(s)| \leq \delta\right)>0 .
$$

PROOF. The proof relies on a similar result for Brownian motion [17], Section 1.4. Let us give some details.

Fix a Lipschitz excursion $f$ and $\delta>0$ as in the statement of the lemma. Without loss of generality, assume that $\delta<1 / 2$. Define $g_{\delta}(t)=\min (\delta, t, 1-t)$, so that $\left\|g_{\delta}\right\|_{\infty}=\delta$. Then

$$
\left|e(s)-f(s)-g_{\delta / 2}(s)\right| \leq \delta / 2 \Rightarrow|e(s)-f(s)| \leq \delta .
$$

Therefore, replacing if necessary $f$ by $f+g_{\delta / 2}$ and $\delta$ by $\delta / 2$, we can assume without loss of generality that the following holds:

$$
\text { for any } \eta \leq \delta / 2 \text { and } s \in[\eta, 1-\eta] \text {, one has } f(s) \geq \eta \text {. }
$$

We extend $f$ to the interval $[-\delta, 1+\delta$ ] by setting $f(t)=t$ if $t \leq 0$ and $f(t)=$ $1-t$ if $t \geq 1$. Let $\left(B_{t}\right)_{t \geq 0}$ be a realization of the Brownian motion. Let $\eta \in(0, \delta / 2]$. Using the results of [17], Section 1.4, we know that with positive probability we have

$$
\sup _{s \in[1 / 2-\delta, 3 / 2+\delta]}\left|B_{s}-f\left(s-\frac{1}{2}\right)\right|<\eta .
$$

Together with (16), this implies in particular that

$$
\begin{cases}B_{s}>0 & \text { if } s \in\left[\frac{1}{2}+\eta, \frac{3}{2}-\eta\right] \\ B_{s}<0 & \text { if } s \in\left[\frac{1}{2}-\delta, \frac{1}{2}-\eta\right] \cup\left[\frac{3}{2}+\eta, \frac{3}{2}+\delta\right] .\end{cases}
$$

Therefore, if we define $a$ and $b$ as in (15), we have

$$
\frac{1}{2}-\eta<a<\frac{1}{2}+\eta, \quad \frac{3}{2}-\eta<b<\frac{3}{2}+\eta .
$$

In particular, $|b-a-1|<2 \eta$. For $s$ in $[0,1]$, we can write

$$
\begin{aligned}
|e(s)-f(s)|= & \left|\frac{1}{\sqrt{b-a}} B_{a+s(b-a)}-f(s)\right| \\
\leq & \frac{1}{\sqrt{b-a}}\left|B_{a+s(b-a)}-f\left(a-\frac{1}{2}+s(b-a)\right)\right| \\
& +\left|\frac{1}{\sqrt{b-a}}-1\right| \cdot\left|f\left(a-\frac{1}{2}+s(b-a)\right)\right| \\
& +\left|f\left(a-\frac{1}{2}+s(b-a)\right)-f(s)\right| .
\end{aligned}
$$


Using (17) and (18), the inequality $|b-a-1|<2 \eta$ and the fact that $f$ is a bounded Lipschitz function, it is not hard to see that this upper bound is smaller than $C \eta$ for some constant $C$. Since $\eta$ can be chosen as small as wanted, we may assume $C \eta \leq \delta$ and we get $|e(s)-f(s)| \leq \delta$ (for all $s$ in $[0,1]$ ).

In summary, for $\eta$ sufficiently small, (17) implies $\sup _{0 \leq s \leq 1}|e(s)-f(s)| \leq \delta$, so that the latter occurs with positive probability, as wanted.

Acknowledgements. Many thanks to Carine Pivoteau for providing Boltzmann samplers of permutations in classes, for running experiments with us using those and for producing the simulations shown in the Introduction. In addition, we thank Dominique Rossin for sharing the results of early experiments he did on random permutations in classes.

We are also very grateful to Grégory Miermont for discussions at several stages of the project; he suggested in particular the exchangeability argument at the core of the proof of Proposition 6.1. We also thank warmly Igor Kortchemski for helping us find our way in the literature on the Brownian motion and excursion. Finally, we thank Douglas Rizzolo for pointing out that the value of the variance in Proposition 2.15 was wrong in the first version of the present paper.

FB, LG and AP also benefited from the hospitality of the Institut für Mathematik of Zürich in May and December 2015.

Finally, we thank an anonymous referee for his/her constructive comments, in particular for pointing out the measurability issue discussed in Section 2.5 and several remarks and bibliographical pointers that allowed us to shorten some proofs.

\section{REFERENCES}

[1] Albert, M., Homberger, C. and Pantone, J. (2015). Equipopularity classes in the separable permutations. Electron. J. Combin. 22 Paper 2.2. MR3359905

[2] Albert, M. H. and AtKinson, M. D. (2005). Simple permutations and pattern restricted permutations. Discrete Math. 300 1-15. DOI:10.1016/j.disc.2005.06.016. MR2170110

[3] Aldous, D. (1993). The continuum random tree. III. Ann. Probab. 21 248-289. MR1207226

[4] ATAPOUR, M. and MADRAS, N. (2014). Large deviations and ratio limit theorems for patternavoiding permutations. Combin. Probab. Comput. 23 160-200.

[5] Avis, D. and Newborn, M. (1981). On pop-stacks in series. Util. Math. 19 129-140. MR0624050

[6] Bassino, F., Bouvel, M., Féray, V., Gerin, L., Maazoun, M. and Pierrot, A. Universal limits of substitution-closed permutation classes. Available at arXiv:1706.08333.

[7] Bevan, D. (2015). On the growth of permutation classes Ph.D. thesis, Open University. Available at arXiv:1506.06688.

[8] Billingsley, P. (1999). Convergence of Probability Measures, 2nd ed. Wiley, New York. DOI:10.1002/9780470316962. MR1700749

[9] Bogachev, V. I. (2007). Measure Theory, Vol. 2. Springer, Berlin.

[10] BónA, M. (2010). The absence of a pattern and the occurrences of another. Discrete Math. Theor. Comput. Sci. 12 89-102.

[11] BónA, M. (2012). Surprising symmetries in objects counted by Catalan numbers. Electron. J. Combin. 19 Paper 62. 
[12] BónA, M. (2012). Combinatorics of Permutations, 2nd ed. Chapman-Hall and CRC Press, London.

[13] Bose, P., Buss, J. F. and Lubiw, A. (1998). Pattern matching for permutations. Inform. Process. Lett. 65 277-283. DOI:10.1016/S0020-0190(97)00209-3. MR1620935

[14] Cheng, S.-E., EU, S.-P. and Fu, T.-S. (2007). Area of Catalan paths on a checkerboard. European J. Combin. 28 1331-1344.

[15] The Sage Developers (2016). Sage mathematics software (Version 7.1). Availabel at http: //www.sagemath.org.

[16] Dokos, T. and PAK, I. (2014). The expected shape of random doubly alternating Baxter permutations. Online J. Anal. Comb. 9 Article 5.

[17] Freedman, D. (2012). Brownian Motion and Diffusion, 3rd ed. Springer, Berlin.

[18] Ghys, E. A Singular Mathematical Promenade. ENS Éditions, Lyon.

[19] Glebov, R., Grzesik, A., Klimosová, T. and Král', D. (2015). Finitely forcible graphons and permutons. J. Combin. Theory Ser. B 110 112-135.

[20] Hoffman, C., Rizzolo, D. and Slivken, E. (2016). Pattern avoiding permutations and Brownian excursion part II: Fixed points. Probab. Theory Related Fields 169 377-424.

[21] Hoffman, C., Rizzolo, D. and Slivken, E. (2017). Pattern avoiding permutations and Brownian excursion part I: Shapes and fluctuations. Random Structures Algorithms 50 394-419.

[22] Homberger, C. (2012). Expected patterns in permutation classes. Electron. J. Combin. 19 Paper 43.

[23] Hoppen, C., Kohayakawa, Y., Moreira, C. G., Rath, B. and Sampaio, R. M. (2013). Limits of permutation sequences. J. Combin. Theory Ser. B 103 93-113.

[24] JAnson, S. (2017). Patterns in random permutations avoiding the pattern 132. Combin. Probab. Comput. 26 24-51.

[25] Janson, S., NAKAmura, B. and Zeilberger, D. (2015). On the asymptotic statistics of the number of occurrences of multiple permutation patterns. J. Comb. 6 117-143.

[26] Kallenberg, O. (2006). Foundations of Modern Probability, Springer, New York.

[27] Kenyon, R., Král', D., Radin, Ch. and Winkler, P. (2015). Permutations with fixed pattern densities (previous title: A variational principle for permutations). Available at arXiv: 1506.02340.

[28] Kitaev, S. (2011). Patterns in Permutations and Words. Springer, Berlin.

[29] KorTCHEMSKI, I. (2012). Invariance principles for Galton-Watson trees conditioned on the number of leaves. Stochastic Process. Appl. 122 3126-3172. DOI:10.1016/j.spa.2012. 05.013. MR2946438

[30] Le Gall, J.-F. (2005). Random trees and applications. Probab. Surv. 2 245-311.

[31] MAAzOUN, M. (2017). On the Brownian separable permuton. In preparation.

[32] Madras, N. and LiU, H. (2010). Random pattern-avoiding permutations. In Algorithmic, Probability and Combinatorics. Contemp. Math. 520 173-194. Amer. Math. Soc., Providence, RI.

[33] Madras, N. and Pehlivan, L. (2016). Structure of random 312-avoiding permutations. Random Structures Algorithms 49 599-631. DOI:10.1002/rsa.20601. MR3545829

[34] Miner, S. and PAK, I. (2014). The shape of random pattern-avoiding permutations. Adv. in Appl. Math. 55 86-130.

[35] Mörters, P. and Peres, Y. (2010). Brownian Motion. Cambridge Univ. Press, Cambridge.

[36] Pitman, J. (2006). Combinatorial Stochastic Processes, 2002 Saint-Flour Lecture Notes. Lecture Notes in Math. 1875. Springer, Berlin.

[37] Pitman, J. and Rizzolo, D. (2015). Schröder's problems and scaling limits of random trees. Trans. Amer. Math. Soc. 367 6943-6969. DOI:10.1090/S0002-9947-201506254-0. MR3378819 
[38] RudolPh, K. (2013). Pattern popularity in 132-avoiding permutations. Electron. J. Combin. 20 Paper 8.

[39] Shapiro, L. and Stephens, A. B. (1991). Bootstrap percolation, the Schröder numbers, and the $n$-kings problem. SIAM J. Discrete Math. 4 275-280.

[40] Vatter, V. (2015). Permutation classes. In Handbook of Enumerative Combinatorics 753833. CRC Press, Boca Raton, FL. MR3409353

[41] Westenberg, M. A., Roerdink, J. B. T. M., Kuipers, O. P. and van Hijum, S. A. F. T. (2010). Random graphs and complex networks. Lecture notes. Available at http://www. win.tue.nl/ rhofstad/.

[42] WIKIPEDIA. Enumerations of specific permutation classes. Available at https://en.wikipedia. org/wiki/Enumerations_of_specific_permutation_classes. Accessed on Jan. 5th, 2016.

F. BASSINO

UNIVERSITÉ PARIS 13

SORBONNE PARIS CITÉ

LIPN, CNRS UMR 7030

F-93430 Villetaneuse

FRANCE

E-MAIL: bassino@lipn.univ-paris13.fr

L. GERIN

CMAP

ÉCOLE POLYTECHNIQUE

CNRS, ROUTE DE SACLAY

91128 PALAiseau CEDEX

FRANCE

E-MAIL: gerin@cmap.polytechnique.fr
M. Bouvel

V. FÉRAY

INSTITUT FÜR MATHEMATIK

UNIVERSITÄT ZÜRICH

WINTERTHURERSTR. 190

CH-8057 ZÜRICH

SWITZERLAND

E-MAIL: mathilde.bouvel@math.uzh.ch valentin.feray@math.uzh.ch

A. PIERROT

LRI, UNIV. PARIS-SUD - CNRS

UNIVERSITÉ PARIS-SACLAY

91405 ORSAY

FRANCE

E-MAIL: adeline.pierrot@1ri.fr 